\title{
Recent Progress in Shape Memory Polymer: New Behavior, Enabling Materials, and Mechanistic Understanding
}

\author{
Qian Zhao ${ }^{1}$, H. Jerry $\mathrm{Qi}^{2}$, Tao Xie ${ }^{1 *}$ \\ ${ }^{1}$ State Key Laboratory of Chemical Engineering \\ Department of Chemical and Biological Engineering \\ Zhejiang University \\ 38 Zheda Road \\ Hangzhou, $310027 \quad$ P. R. China \\ ${ }^{2}$ The George W. Woodruff School of Mechanical Engineering \\ Georgia Institute of Technology \\ 801 Ferst Drive \\ Atlanta, GA 30332 USA
}

\begin{abstract}
Shape memory polymers (SMPs), as a class of programmable stimuli-responsive shape changing polymers, are attracting increasing attention from the standpoint of both fundamental research and technological innovations. Following a brief introduction of the conventional shape memory effect (SME), progress in new shape memory enabling mechanisms and triggering methods, variations of in shape memory forms (shape memory surfaces, hydrogels, and microparticles), new shape memory behavior (multi-SME and two-way-SME), and novel fabrication methods are reviewed. Progress in thermomechanical modeling of SMPs is also presented.
\end{abstract}

Keywords: shape memory polymer, smart materials, stimuli-responsive polymer, polymer modeling

*Corresponding author: Email: taoxie@zju.edu.cn 


\begin{tabular}{|c|c|}
\hline \multicolumn{2}{|c|}{ Abbreviations: } \\
\hline $\mathrm{SCPs}$ & shape changing polymers \\
\hline LCEs & liquid crystalline elastomers \\
\hline SMP & shape memory polymer \\
\hline SME & shape memory effect \\
\hline $2 \mathrm{~W}$ & two-way \\
\hline $1 \mathrm{~W}$ & one-way \\
\hline$T_{\text {trans }}$ & transition temperature \\
\hline$T_{\mathrm{g}}$ & glass transition temperature \\
\hline$T_{\mathrm{m}}$ & melting temperature \\
\hline$T_{\mathrm{cl}}$ & liquid crystal cleaning temperature \\
\hline$T_{\mathrm{d}}$ & deformation temperature \\
\hline$T_{\mathrm{f}}$ & shape fixing temperature \\
\hline$T_{\mathrm{c}}$ & crystallization temperature \\
\hline$R_{\mathrm{f}}$ & shape stability ratio \\
\hline$R_{\mathrm{r}}$ & shape recovery ratio \\
\hline$T_{\mathrm{sw}}$ & switching temperature \\
\hline$\varepsilon_{\max }$ & maximum recoverable strain \\
\hline$\sigma_{\max }$ & maximum recovery stress \\
\hline $\mathrm{SMC}$ & shape memory cycle \\
\hline$\varepsilon_{\text {load }}$ & strain under load \\
\hline$\varepsilon$ & fixed strain \\
\hline$T_{\mathrm{r}}$ & recovery temperature \\
\hline$\varepsilon_{\mathrm{rec}}$ & recovered strain \\
\hline$V_{\mathrm{r}}$ & strain recovery rate \\
\hline$T_{\sigma \max }$ & temperature corresponding to $\sigma_{\max }$ \\
\hline$T_{\text {sw,app }}$ & apparent switching temperature \\
\hline PCL & poly $(\varepsilon$-caprolactone $)$ \\
\hline SMPU & shape memory polyurethane \\
\hline EMU & elemental memory unit \\
\hline TME & temperature memory effect \\
\hline PU & polyurethane \\
\hline$T_{\mathrm{i}}$ & liquid-crystal isotropic transition temperature \\
\hline$T_{\mathrm{v}}$ & vitrification temperature \\
\hline CIE & crystallization induced elongation \\
\hline MIC & melting induced contraction \\
\hline
\end{tabular}




\section{Contents}

1. Introduction

2. General aspects of SMPs

2.1. Programming

\subsection{Classification}

2.3 Basic molecular requirements and mechanism

2.4 SMP Characterization

3. Shape memory switches and triggers

3.1 Phase versus molecular switches and thermal versus non-thermal triggers

3.2 Direct versus indirect trigger methods

4. Variants in shape memory forms

4.1 Shape memory surfaces

4.2 Shape memory hydrogels

4.3 Shape memory microparticles

5. Multi-SMPs

5.1 Intrinsic multi-SMPs

5.2 Extrinsic multi-SMPs

5.3 Reprocessible multi-SMPs

6. Two-way SMPs

6.1 Quasi 2W-SME for liquid crystalline elastomers

6.2 Quasi 2W-SME for semi-crystalline networks

6.3 2W-SME for semi-crystalline networks

7. Novel SMP fabrication methods

7.1 Electrospinning

7.2 Four dimension printing

7.3 Photo-induced shape transformation

7.4 Origami-inspired manufacturing

8. Modeling of thermomechanical behavior of SMPs

8.1 Thermoviscoelastic models

8.2 Phase evolution models

8.3 Models for predicting shape memory performance

9. Summary and outlook

Acknowledgment

References 


\section{Introduction}

Active shape change in response to environment conditions is commonplace in nature [1]. In fact, this property is essential for the survival of animals and even some plants. Among numerous interesting examples are sunflowers that move with the sun to maximize light exposure, the opening and closing of Venus flytrap to capture preys, and the microscopic shape changes in the skin layers of cattlefish that enables its macroscopic color change.

Polymer materials, in their early days, were mostly studied for use as static structural parts. In the modern days, however, advanced polymer materials that exhibit special functions in response to external conditions have attracted more and more attention. Such behavior is similar to the biological intelligence observed in nature. Accordingly, such polymers are also called smart polymers, or in a more scientific term, stimuli-responsive polymers. Whereas macroscopic changes in polymer functions are often accompanied by chain conformational changes (i.e., shape changes at the molecular scale), stimuli-responsive shape changing polymers (SCPs) commonly refer to those for which shape changes are either macroscopic or at least visible under microscopes. Examples of stimuli-responsive SCPs are numerous, ranging from the well known swelling/deswelling of hydrogels [2] to the intriguing reversible surface morphological changes of liquid crystalline elastomers (LCEs) [3]. Of importance here is that, for the preceding two examples, after the materials are fabricated, the shape change can only occur between a fixed number of equilibrium shapes (often two). That is, their shape shifting cannot be manipulated externally after fabrication, a non-programmable shape changing behavior.

In contrast, shape memory polymers (SMPs) are a class of stimuli-responsive SCPs for which shape shifting behavior can be programmed. This is the single most important feature that distinguishes SMPs from other stimuli-responsive SCPs. Specifically, a typical SMP can be programmed to fix one temporary shape and subsequently recover to its permanent shape upon stimulation (typically heating), as illustrated in Fig. 1a. Here, the temporary shape is usually defined by the applied force during the shape fixing step. Accordingly, this step is also called a programming step. Since the shape memory cycle in Fig. 1a involved a total of two shapes (one temporary and one permanent), the associated behavior is called a dual-shape memory effect (dual-SME), representing the simplest and most well known behavior for SMPs.

From a molecular structure standpoint, the dual-SME is enabled by the combination of a reversible switching mechanism and a network structure [4]. Thus, the simplest (and very common) polymer systems, such as a crosslinked (chemically or physically) amorphous (or crystalline) polymer would exhibit such a behavior [5-10]. Indeed, the dual-SME has been known for quite a long time and is the basis of numerous technological innovations. Best known examples of commercial applications include heat-shrinkable tubes used in cable industry, heat-shrinkable labels used for packaging, and shrink-dink toys. Other high value-added applications, mostly in the biomedical [11-15] and aerospace fields [16-18], are also being actively explored. The vast potential to enable technological innovations highly relevant for the human society (e.g., health) has been the driving force for active research on SMPs. 
Broadly speaking, current SMP research can be classified into the following focus areas:

(1). exploration of new and exciting applications;

(2). tuning SMP molecular structures to meet application specific requirements;

(3). alternative stimulation mechanisms that are relevant for the intended application;

(4). new molecular designs beyond the simplest SMP system mentioned in the preceding;

(5). discovery of new shape memory behavior (or effects).

\section{a}

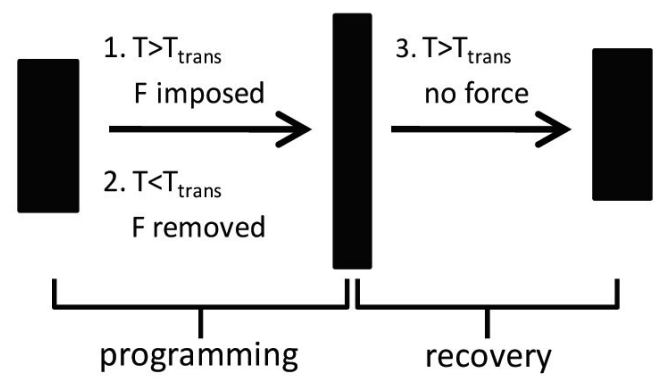

b

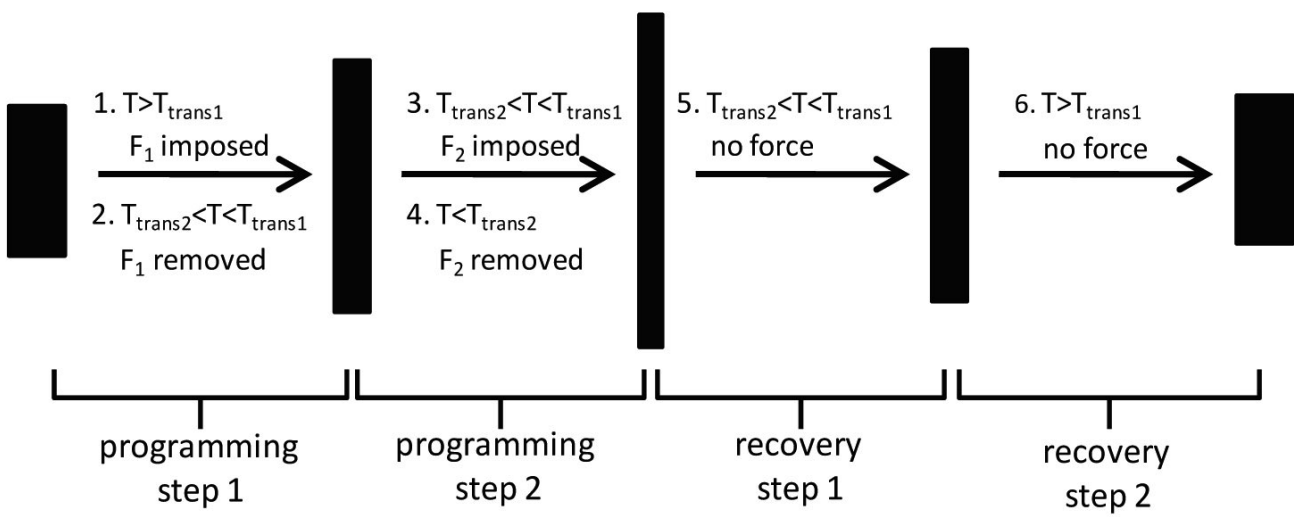

Fig. 1. Schematic illustrations of (a). Dual-shape memory effect; and (b). Triple-shape memory effect.

Among these focus areas, the first one is perhaps most important over the long term. This is simply due to the proven fact that any research field can only thrive with promises in impacting technological innovations. Within this big umbrella (focus area 1), focus areas 2-4 have been the routine parts of SMP research. Such a statement does not discredit their importance. In fact, without 2 and 3, any potential application ideas would not advance beyond the conceptual stage. Focus area 4 is not just scientifically interesting, but could contribute to all the other focus areas. For instance, physical doping of crystalline small molecules into an elastomer network opens up a simple way to tune the transition temperature of SMPs [19]. The discovery of new shape memory behavior (Focus area 5), by contrast, happens very rarely. Yet, new shape memory behavior could lead to new application opportunities in ways that are much more drastic than the others. As such, there are two notable achievements during the past decade in SMPs: multi-shape memory polymers (multi-SMPs) and two-way shape memory polymers (2W-SMPs).

A multi-SMP refers to a polymer that is capable of "memorizing" more than one temporary shape and subsequently recovering in a highly controllable manner. Notably, this definition of multi-SMPs makes no distinction between the so-called triple-SMP (Fig. 1b) and an SMP with 
more than two temporary shapes. It is critical to point out that, in both Fig. 1a and 1b, the shape changing occurs only in one direction, as the arrows indicated. That is, temperature change alone would not drive the recovered shape(s) back to the temporary shape(s). Importantly, this "irreversibility" should be distinguished from reprogramming in that change in both temperature and external stress condition would enable the switch from the recovered shape to the temporary shape. Thus, both the dual- and multi-SMEs discussed in the preceding belong to the so-called one-way (or irreversible) shape memory effect (1W-SME). In contrast, a $2 \mathrm{~W}-\mathrm{SMP}$ can exhibit programmable and reversible shape switching between two (or more) distinct shapes. Here again, "programmable" is the key that distinguishes a 2W-SMP from many common examples of reversible SCPs. Giving the importance of the programmability, we will elaborate it more further in the following.

It is noted here that many excellent reviews have been published by various groups active in SMP research. These reviews cover general aspects of SMPs [4-10], multi-functional SMPs [20], SMP composites [21-26], SMP foams [27], SMP fibers [28-30], SMP characterization [31], and SMPs for biomedical [12-15] and aerospace applications [16-18]. Readers interested in specific topics are referred to these reviews accordingly. Although some general aspects of SMPs will be necessarily covered, the main objective of this review is to highlight notable progress made, mostly in the last five years, clarify some conceptual confusion within the field, and point out unanswered questions (opportunities). The structure of the review is organized as following: general aspects of SMPs, shape memory switches and triggers, variants in SMP forms, multi-SMPs, 2W-SMPs, SMP fabrication methods, and SMP modeling.

\section{General aspects of SMPs}

\subsection{Programming}

From the literal meaning of the term "shape memory polymer", most researchers would agree that an SMP should possess the ability to transform from any deformed temporary shape back into a "memorized" shape (i.e., permanent or original shape). This seemingly simple description, however, may cause confusion in the larger context of SCPs. As stated earlier, SMPs are a sub-class within SCPs for which shape shifting is programmable. Thus, the fundamental issue lies in the definition of programming (or programmability). Programming refers to the external and physical manipulation process that defines the shape shifting pathway. This process should be independent of material synthesis (or fabrication). Using a dual-SMP shown in Fig. 1a as an example, it is the thermomechanical shape fixing (programming) that determines the temporary shape, thus the shape shifting pathway goes from this particular shape to its original shape. Importantly, since programming is external and physical, for the same material, countless temporary shapes (thus pathways) are possible by changing the programming conditions (e.g., the deformation force) (shown in Fig. 2a) [32]. Of course, within each shape memory cycle, one has to pick one particular shape shifting pathway for the SMP to perform, but different pathways can be implemented in other shape memory cycles. Similarly, for a $2 \mathrm{~W}-\mathrm{SMP}$ system, the same sample (Fig. 2b) can be programmed to change from shape A and shape B and reprogrammed to switch between shape A' to shape B', in a reversible fashion [33]. This "programmability" clearly distinguishes SMPs from other 
forms of SCP. As an example of a non-programmable SCP, a thermo-sensitive hydrogel such as poly(N-isopropylacrylamide) can undergo expansion-shrinking shape transformation (Fig. 2c) upon swelling and deswelling induced by an environmental change [2,34]. Another example is the temperature induced surface morphological change of a liquid crystal elastomer (LCE) (Fig. 2d) [3]. In both cases, the shape shifting is between two fixed states defined in the material fabrication step. Adding a bit confusion to the issue is that although the two examples in Fig. 2c and Fig. 2d did not show programmability, hydrogels and LCEs can both be designed to introduce programmability, thus qualifying them as SMPs. Examples of such can be found later in this review.
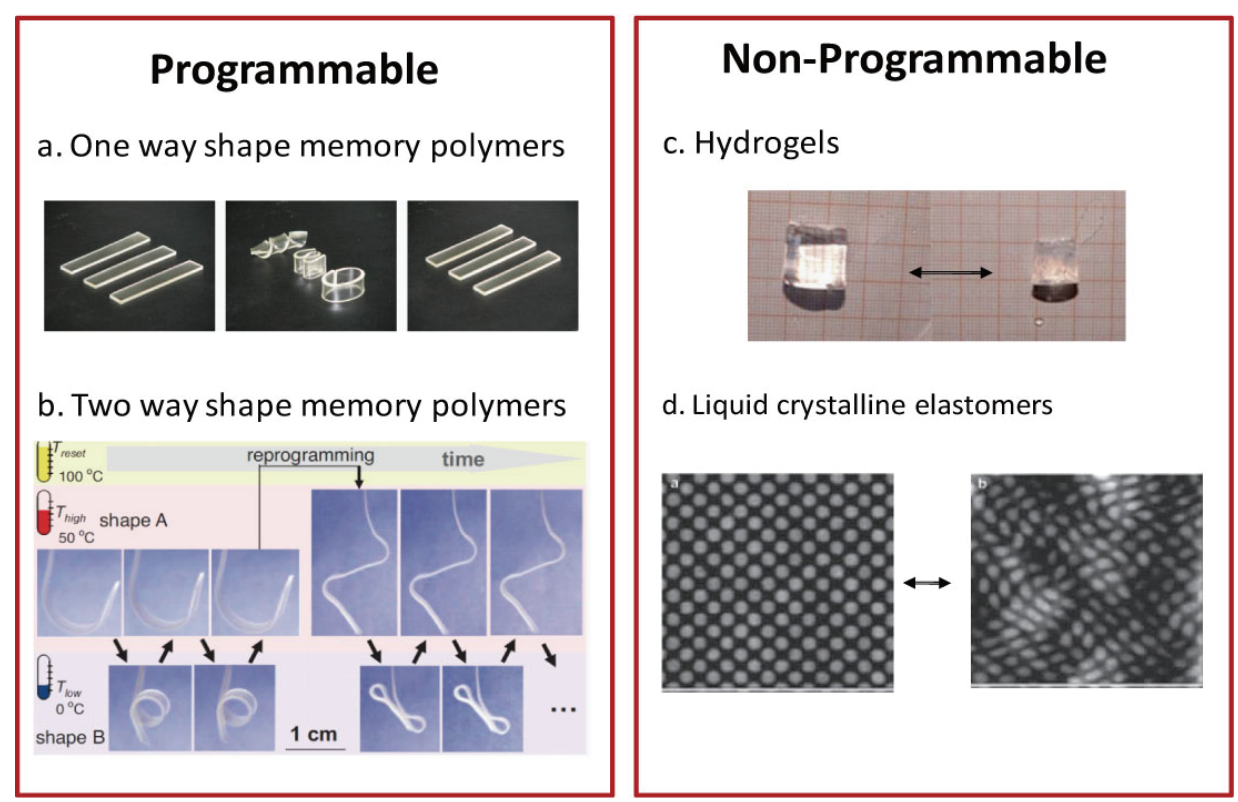

Fig. 2. Classification of shape changing polymers: (a). One way shape memory polymers [32], Copyright 2009. Reproduced with permission from Elsevier Ltd; (b). Two way shape memory polymers [33], Copyright 2013. Reproduced with permission from John Wiley and Sons; (c). Hydrogels [34], Copyright 2012. Reproduced with permission from Royal Society of Chemistry; (d). Liquid crystalline elastomers [3], Copyright 2006. Reproduced with permission from the American Chemical Society.

It is worth pointing out that the term "program (or programming)" has been used in a broader sense in the context of SCPs. For instance, Hayward's group developed a half tone lithography method to introduce material heterogeneity into a hydrogel sheet that can undergo highly complex shape changing [35]. The term "program" could be used there to describe the lithography process that defines the shape shifting. Since such programming is an integral part of the fabrication process, the material after fabrication can only switch between fixed shapes, no matter how sophisticated they are. Likewise, our group has recently reported a "Lego" hydrogel system for which shape-changing behavior could be mechanically programmed by prestretching hydrogel building blocks during the material assembly step [36]. But again, the "programming" occurs in the material fabrication step, fundamentally different from the external and physical nature of the programming for SMPs. 
With the clear definition of "programming", SCPs can be readily classified as programmable SCPs (i.e., SMPs) and non-programmable SCPs (Fig. 2). We should note that Lendlein's group uses the same definition for SMPs, however, they use "actively moving polymers" to describe the general class of SCPs used in our definition. This allows them to reserve the term "SCPs" for the non-programmable SCPs in our definition. This difference in terminology is purely a matter of preference and, in our case, we simply elect to use the literal meaning of shape changing polymers to describe SCPs.

\subsection{Classification}

The simplest and also the most common SMP is a thermally induced one-way dual-SMP in its neat polymer form. Among the attributes, "thermally induced" refers to the external stimulation method (or so-called trigger) that is most often employed to induce shape recovery. "One-way" implies that the shape recovery is irreversible. That is, shape shifting during recovery can only follow the pathway from a temporary shape to the permanent shape, but not vice versa. "Dual-shape" means that such a shape memory cycle involves only two shapes: a metastable temporary shape and an equilibrium permanent shape. Both the terms "one-way" and "dual-shape" refer to the shape shifting behavior of the materials. Accordingly, non-traditional SMPs include non-thermally induced SMP, 2W-SMPs, and multi-SMPs. Any combination of these three defining attributes can be used to the categorize SMPs. An example of such is a non-thermally induced one-way multi-SMP. In addition, an SMP may also be in a non-neat polymer form such as a hydrogel or a composite [6,21]. As shall be seen, these variations from the simplest form of SMP provide the basic logic for this review.

Traditionally, SMPs have been categorized into chemically (or physically) crosslinked glassy (or semi-crystalline) polymers [4,5]. This classification emphasizes chemical structures and morphologies that are more closely related to the molecular designs of SMP. We note that such a classification is only valid for conventional SMP relying on thermal phase changes, called phase switches in this review. Increasingly, SMP enabling mechanisms called molecular switches [9], originate from reversible molecular interactions instead of reversible phases are becoming more popular. Examples are discussed extensively in a dedicated section in this review. Accordingly, SMPs operating via various molecular switching mechanisms represent nonconventional molecular design of SMP.

\subsection{Basic molecular requirements and mechanism}

Here we take the baseline case, i.e., a thermally induced one-way dual-SMP with phase switches, for the discussion of the basic molecular requirements and general mechanism for SMPs. The mechanism for other more sophisticated forms of SMPs will be deduced accordingly and presented in the coming sections. Figure 3 shows a typical thermally induced one-way dual-shape memory cycle. At room temperature, the SMP is typically a rigid plastic. Upon heating above its shape memory transition temperature $T_{\text {trans }}$, the material becomes a soft rubber (or elastomer) due to the increased mobility of the molecular chains. Here, $T_{\text {trans }}$ can be a glass transition temperature $T_{\mathrm{g}}$, a melting temperature $T_{\mathrm{m}}$, or less commonly liquid crystal clearing temperature $T_{\mathrm{cl}}$. Heating allows easy deformation when an external force is applied. 
The associated elevated temperature is called $T_{\mathrm{d}}$ (deformation temperature). In an ideal situation, this macroscopic deformation should correspond to changes in the molecular chain conformation, or entropy. When cooled to a temperature below the $T_{\text {trans }}$, the deformation imposed onto the SMP will be maintained even after the removal of the external force. At the molecular level, this is simply due to the freezing of the molecular chain (i.e., reduction of chain mobility) that locks in the deformed chain conformation, or enables the storage of entropic energy in the system. The corresponding temperature at this stage is called $T_{\mathrm{f}}$ (shape fixing temperature). This temporary shape remains stable unless when it is heated above the $T_{\text {trans }}$ again in the absence of external stress. Under this condition, the SMP will recover to its permanent shape. Again at the molecular scale, heating activates the molecular mobility, which releases the entropic energy, thus driving the molecular chains back into its highest entropic state corresponding to the permanent shape.

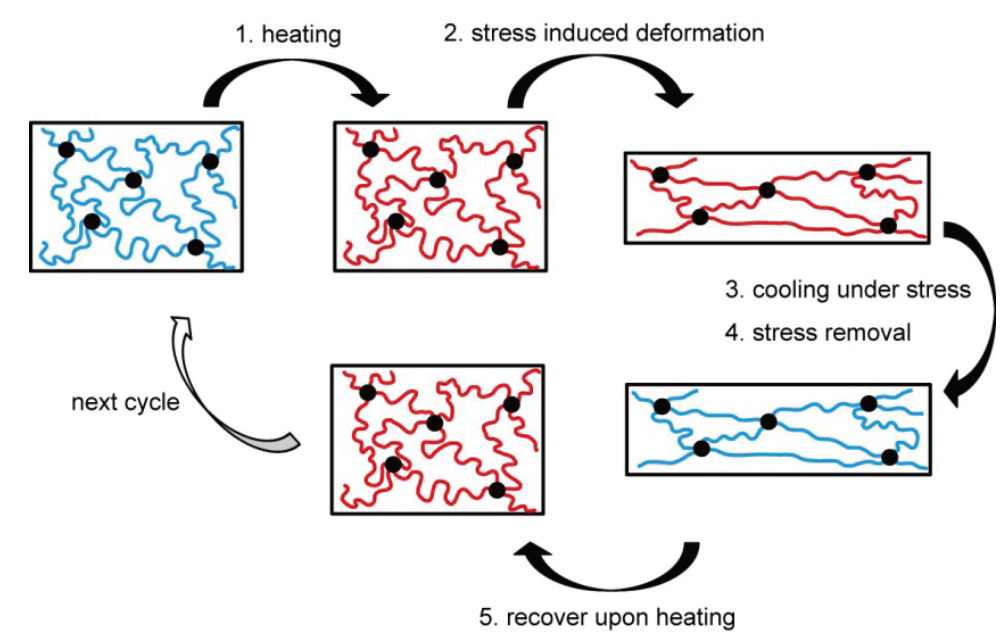

Fig. 3. The molecular mechanism of the dual-SME. Black dots: netpoints; blue lines: molecular chains of low mobility below $T_{\text {trans }}$; red lines: molecular chains of high mobility above $T_{\text {trans. }}$.

The preceding discussion suggests that polymer shape memory effect is an entropic phenomenon. From the molecular requirement standpoint, what is readily clear is the need for molecular chains for which mobility can be activated and depressed, typically by a temperature change. Given its switchable nature, we called these switch segments. Here, the term segments instead of chains are used to signify that the switching does not necessarily have to occur to the entire chain, but can happen at the finer segmental level. In Fig. 3, the color change for all the chains illustrates the mobility switching, but it is important to understand this is done for ease of demonstration. In reality, the switching may only happen within certain segments along the chain. A point that needs to be emphasized is that, whereas the schematics shown in Figure 3 shows free space between chains, it is for ease of demonstration only. In reality, this type of free space (volume) is not necessary since chain conformation change typically occurs in a highly coordinate manner to satisfy the spatial requirements. That is, the chains or segments that are not responsible for conformation change would move at the system level to accommodate conformation changes within the switching segments, even though they 
themselves do not necessarily undergo conformation changes.

The segmental switching alone is not sufficient for polymer shape memory effect. Another mechanism has to be in place to ensure that any macroscopic deformation is the results of molecular chain conformation change, that is, deformation should lead to an entropic change that is the necessary driving force for recovery. To satisfy this requirement, the system has to possess a mechanism to ensure that there is no slippage of entire polymer chains that would lead to macroscopic deformation but no entropic change. Such a mechanism is provided by the network established by the black netpoints shown in Fig. 3 .

Overall, an SMP should meet two structural requirements: one is the netpoints that defines the permanent shape and the other is the switch segments that can enable fixation of a temporary shape. The netpoints signify that polymer chains within an SMP form a network structure. In the permanent shape, the molecular chains within the SMP network are at the lowest energy (highest entropy) state. As long as the network is stable, any macroscopic deformation imposed on the permanent shape would change the conformation of the polymer chains and raise the energy state of the materials. At this point, it is the cooling induced depression of the molecular mobility that serves as the kinetic trap to maintain the system in the high energy state. Once this kinetic trap is removed by reactivating the segmental mobility (e.g., by heating), the entropic energy is released, driving the molecular chains back to their lowest energy (highest entropy) state, thus the materials would accordingly recover the original shape. Here, the netpoints are either chemical or physical crosslinking points or phases. They are the mechanism that ensures storage and release of entropic energy that is responsible for the shape fixing and recovery of an SMP. The netpoints should be sufficiently stable to withstand the thermo-mechanical conditions encountered in the shape memory process without breakage; otherwise the permanent shape would not fully recover. The extent of shape recovery is thus a direct reflection of the stability of the network established by the netpoints under the relevant conditions.

The netpoints can be made up of either chemical covalent bonds or physical interactions (e.g., crystallites, glassy hard domains [5], hydrogen bonding [37], ionic assembly [38-40], and chain entanglements [41]). Whereas we use the term "netpoints" here, it should be noted that the netpoints are not necessarily points per se, but the term is used mostly for lack of better words. For chemically crosslinked system, the network is indeed established by the atomic netpoints each of which connects more than two chains together. For many physically crosslinked SMP systems such as thermoplastic polyurethane SMPs, the netpoints are microscopic phases or domains corresponding to the hard segments. Interesting variants are the so-called nanoparticle tethered SMP systems with linear polymer chains grafted onto nanoparticles [37,42-44]. For this type of systems, the nanoparticles alone do not establish the necessary network since the other ends of the grafted chains remain free. When combined with chemical crosslinks or physical entanglement, however, a network structure is formed with the nanoparticles serving as the netpoints in conjunctions with chemical crosslinking points or entanglement. Here, of course, the nanoparticle netpoints are neither atomic points nor microscopic phases. What is critical here is not the scale of the netpoints, but that they establish a stable network within the polymer. 
Since the network (or netpoints) defines the permanent shape, often time the term "permanent" is also extended to describe the network. Such a "permanent" network is only of relative meaning. That is, it is ideal that it is permanent (non-breakable) within a particular shape memory cycle. Under conditions different from shape memory cycles, the capability of reversibly forming the "permanent" netpoints implies the SMPs can be re-processed into a new permanent shape. Physical permanent netpoints usually endow the polymers with reprocessability. However, many examples of physical netpoints are not as robust as chemical netpoints. That is, they are more likely to undergo unintended changes in a shape memory cycle, that would lead to incomplete shape recovery. In contrast, the robustness of most chemical netpoints is responsible for the superior shape recovery performance for those systems, but they are typically non-reprocessable. An interesting approach is the use of reversible covalent bonds such as Diels-Alder moieties as the netpoints for SMPs, leading to reprocessable SMPs with good shape recovery performance [45-47].

Typical switch segments are those related to reversible thermal phase transitions (e.g., glass transition, melting-crystallization transition, liquid crystal clearing transition). Since most polymers possess either a glass transition or a melting transition, these two transitions serve as the main backbones for designing SMP. Several differences between glassy SMP and crystalline SMP are noteworthy: 1). Tunability of the transition temperature: $T_{\mathrm{g}}$ for a given SMP class is more readily tunable in a large temperature range via the introduction of comonomers. This has been demonstrated nicely for epoxy SMP $[32,48]$ and meth(acrylate) SMP [49] systems. In contrast, tuning a crystallization temperature in a similar fashion is much harder as the introduction of too much comonomers would destroy the crystallizability of the system all together; 2). Shape memory cycle time: since crystallization is a much slower process than vertrification, a $T_{\mathrm{m}}$-type SMP may require longer time for fixation; 3). Shape memory temperature range: there is often a c.a. 20 degree difference between $T_{\mathrm{m}}$ and $T_{\mathrm{c}}$ (crystallization temperature), the temperature range required for a $T_{\mathrm{m}}$-type SMP is often time larger; and 4). Diversity of the shape memory behavior: as will be discussed later in more detail, a $T_{\mathrm{m}}$-type SMP, along with a liquid crystalline-type SMP, is capable of a $2 \mathrm{~W}$-SME. This is a significant advantage over $T_{\mathrm{g}}$-type SMPs. Besides the two general types of thermal reversible mechanisms, other switch mechanisms have also been explored. The alternative switch mechanisms, while less general for polymers, are important enablers for non-traditional stimulation methods. This will be elaborated further in a dedicated section.

\subsection{SMP Characterization}

Performance parameters for an SMP are diverse; with the most essential ones being the shape memory transition temperature $T_{\text {trans }}$, shape stability ratio $R_{\mathrm{f}}$ and shape recovery ratio $R_{\mathrm{r}}$. Depending on the circumstance (e.g., the intended applications), maximum recoverable strain $\varepsilon_{\max }$ and maximum recovery stress $\sigma_{\max }$ may also be relevant. When the recovery speed is of concern, average and instantaneous recovery rates can also be calculated. The switching temperature $T_{\mathrm{sw}}$, closely related to, but different from, $T_{\text {trans }}$ is also sometimes reported.

Here, $T_{\text {trans }}$ is commonly equal to $T_{\mathrm{g}}$ for an amorphous SMP or $T_{\mathrm{m}}$ for a crystalline SMP, that can be measured using standard thermal analysis methods such as DSC or DMA. $R_{\mathrm{f}}$ and $R_{\mathrm{r}}$ 
can be conveniently determined by manually measuring sample dimension changes by performing a shape memory cycle using a thermal oven according to the steps outlined in Fig. 3. Maximum recoverable strain $\varepsilon_{\max }$, on the other hand, may be best evaluated using a mechanical tester equipped with a thermal chamber by elongating the sample to its failure at the relevant temperature. Multiple measurements are necessary due to the large standard variations inherent in this type of measurements. Traditionally, a $\varepsilon_{\max }$ value much larger than that of shape memory alloys has been perceived as one of the major advantages of SMPs. However, many SMP applications do not require very high $\varepsilon_{\max }$. Despite such, it is important to note that SMPs with $\varepsilon \max _{\max }$ above $800 \%$ are known in the state of art $[41,50]$.

Precise measurement of $R_{\mathrm{f}}$ and $R_{\mathrm{r}}$, determination of $T_{\mathrm{sw}}$ and maximum recovery stress $\sigma$ $\max$, and evaluation of shape recovery rates, are best accomplished by running a quantitative shape memory cycle (SMC) using a thermo-mechanical analyzer. This type of test records precisely the time evolution of temperature, stress, and strain in a SMC. An example of a typical dual-SMC is shown in Fig. 4a. Except its more quantitative nature, this dual-SMC is identical to the qualitative demonstration shown in Figure 1a. The sample is first heated to a deformation temperature $T_{\mathrm{d}}$, normally (but not always) above $T_{\text {trans }}$, a force is then applied as reflected in the stress curve. Upon subsequent cooling under the load to $T_{\mathrm{f}}$, usually below $T_{\text {trans }}$, the sample reaches the so-called strain under load $\varepsilon_{\text {load. }}$. The stress is then removed with the sample strain responding accordingly to a fixed strain $\varepsilon$. This completes the fixing (or programming step). Under a stress free condition, recovery is accomplished by simply heat the sample to a recovery temperature $T_{\mathrm{r}}$, leading to a recovered strain $\varepsilon_{\text {rec }} . R_{\mathrm{f}}$ and $R_{\mathrm{r}}$ can be readily calculated y using Eqs. (1) and (2) below:

$$
\begin{aligned}
& R_{\mathrm{f}}=100 \% \times \varepsilon / \varepsilon_{\text {load }} \\
& R_{\mathrm{r}}=100 \% \times\left(\varepsilon-\varepsilon_{\text {rec }}\right) / \varepsilon
\end{aligned}
$$

Alternatively, an SMC may also be presented in a 3D diagram shown as Fig. 4b using the same set of data. The three axes here are temperature, stress, and stress. Notably absent is the time information. This absence does not preclude the determination of $R_{\mathrm{f}}$ and $R_{\mathrm{r}}$. As such, the use of this type of $3 \mathrm{D}$ shape memory diagram is quite popular in the literature. We caution, however, that the loss of time information may be quite detrimental for more sophisticated SMCs. For instance, if the sample is, at any stage, annealed under a constant stress, the annealing time and whether or not the strain reaches equilibrium during annealing would not be known.
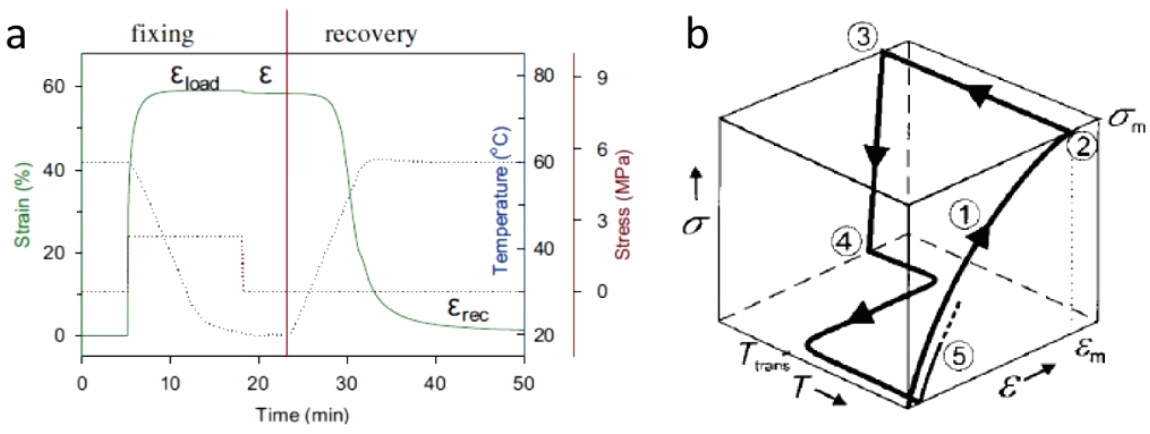

Fig. 4. Quantitative dual-shape memory cycles: (a). 2D diagram [163], Copyright 2013. Reproduced with permission from Nature Publishing Group; and (b). 3D diagram [5], Copyright 2010. Reprinted with permission from John Wiley and Sons. 
The strain curve in the recovery portion of the SMC shown in Fig. 4a can be used to calculate instantaneous strain recovery rate $V_{\mathrm{r}}$, the time derivative of the strain as defined in Eqn. (3). Plotting $V_{\mathrm{r}}$ against temperature as is shown in Figure 5a, one can obtain $T_{\mathrm{sw}}$, defined as the temperature corresponding to the maximum $V_{\mathrm{r}}$.

$$
V_{\mathrm{r}}=\frac{\partial \varepsilon}{\partial \mathrm{t}} \times 100 \%
$$

We note that the SMC in Fig. 4a is obtained under a stress controlled condition in a tensile mode. This is a typical way for constructing an SMC. The type of deformation mode may vary and is not critical in most cases. For instance, a bending mode may also be employed particularly for samples for which $\varepsilon_{\max }$ is relatively small. Under such a circumstance, the bending angles at the corresponding stages are used in place of tensile strains to calculate $R_{\mathrm{f}}$ and $R_{\mathrm{r}}$ in a similar fashion. Alternative to the stress controlled condition, the SMC may also be run under a strain controlled condition. That is, during the programming stage, the tester imposes a certain strain onto the sample while the stress changes accordingly. Another variation lies in the recovery step. Whereas the recovery in Fig. 4a is run under zero-stress for strain recovery (i.e., stress-free strain recovery), it may also be operated under an iso-strain condition. Under such a condition, the tester holds the strain constant, but the tendency of the sample to recover generates a stress called a recovery stress $\sigma$. This is called an iso-strain stress recovery, run to obtain the $\sigma_{\max }$ value. The temperature corresponding to $\sigma_{\max }$ is called $T_{\sigma_{\max }}$, in a way similar to $T_{\mathrm{sw}}$.
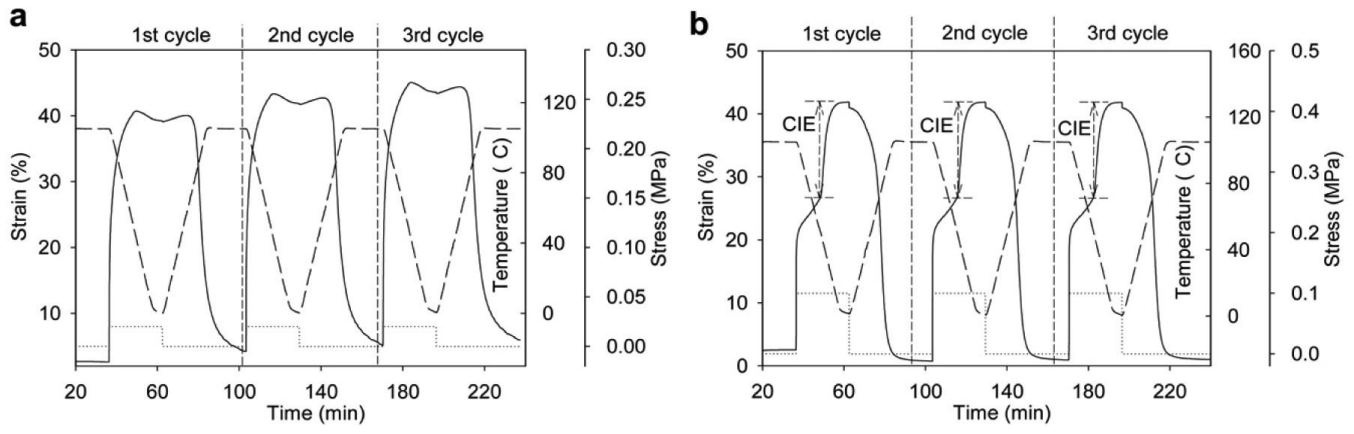

Fig. 5. Consecutive 1W-SMCs of crosslinked EVA with: (a). A low gel fraction; and (b). A high gel fraction. Solid line: strain, dash line: temperature, dot line: stress [51], Copyright 2011. Reproduced with permission from Elsevier Ltd.

Besides a single cycle evaluation, often time it is also necessary to run a cycling experiment to probe the robustness of the shape memory performance over multiple consecutive cycles. This type of cycling experiment is particularly relevant for SMPs intended for device applications. The essence and necessity of the cycling experiment are illustrated in Fig. 5. Figures 5a and 5b correspond, respectively, to two crosslinked EVA (ethylene-vinyl acetate copolymer) samples [51]. The only difference between the two samples lies in the degree of crosslinking, thus the gel fraction. Despite the low gel fraction (48\%) for sample $\mathbf{A}$ in Fig. 5a, its $R_{\mathrm{f}}$ and $R_{\mathrm{r}}$ are about $95 \%$ as determined from the first SMC. Although sample $\mathbf{B}$ in Fig. $5 \mathrm{~b}$ (gel fraction of $76 \%$ ) shows higher $R_{\mathrm{f}}$ and $R_{\mathrm{r}}$ values ( $98 \%$ for both), such a difference 
may appear trivial. However, cycle to cycle comparisons for both samples reveals important difference, that is, there is a notable cycle to cycle strain shift for sample $\mathbf{A}$, whereas such a shift is notably absent for sample B. Clearly, this difference in cycling performance is critical for device applications involving multiple cycles.

Other performance parameters or variations to the SMC conditions are possible, particularly for shape memory experiments that are more sophisticated than the simple dual-SMC in Fig. 4a. While it is impossible to discuss all the possibilities, one only needs to keep in mind that the principles provided in the preceding remain the same, or at least similar. Here, proper selection of SMC conditions requires good understanding of polymer shape memory behavior and what performance parameters a researcher intends to evaluate. In the following section, we shift our focus to discuss the switching and triggering mechanisms that enable polymer shape memory behavior.

\section{Shape memory switches and triggers}

\subsection{Phase versus molecular switches and thermal versus non-thermal triggers}

Early discussion on the molecular origin of polymer shape memory effect revealed that a conventional SMP relies on a reversible thermal phase transition to switch off/on the molecular mobility so as to freeze/release the temporary shape. Naturally, the associated SME is typically thermally activated via direct or indirect heating. One notable exception is the water triggered SMP in which water molecules plastize the amorphous phase, thus reducing the $T_{\mathrm{g}}$ to a level below the ambient temperature to trigger the recovery [52]. The typical need for temperature changes to realize an SME can be fulfilled in many SMP applications, but this also represents a hurdle to realize its full practical potential for instance in situations for which heating is not feasible. On this point, new switching mechanisms that do not rely on thermal phase transitions may offer attractive solutions. Thanks to the development of reversible covalent and non-covalent chemistry, opportunities for designing switch segments at the molecular level (i.e., molecular switches) have emerged. While phase transitions are by and large related to a thermal effect, molecular switches bring more options in terms of triggering mechanisms (e.g., thermal, light, redox, and $\mathrm{pH}$ ).

A prime example of of a non-thermal trigger was reported in 2005 by Lendlein et al. [53]. Photo-reversible [2+2] cycloaddition of cinnamate (or coumarin) groups were introduced into a polymer as the switch mechanism. A deformed sample was exposed to UV light $(\lambda>260 \mathrm{~nm})$ to promote cycloaddition of the cinnamate groups to form crosslinks to lock in the temporary shape. Subsequently, shape recovery could be triggered upon irradiation of UV with $\lambda<260$ $\mathrm{nm}$, as the result of the cleavage of the crosslinks. The non-thermal triggering method has attracted attention, but some practical issues have kept it from becoming popular. The cinnamate-based SMP showed limited $R_{\mathrm{f}}(30 \sim 50 \%)$ and slow respond speed $(\sim 1 \mathrm{~h})$. Subsequent efforts by other groups have improved the shape memory performance to a certain extent [54-56].

By contrast, Lee and coworkers [57-59] reported a photo-responsive SMP system with an entirely different operating mechanism. Instead of relying on photo-reversible covalent chemistry, isomerization of azobenzene was explored as the switching mechanism. This 
particular SMP is a crosslinked glassy liquid crystalline polymer containing azobenzene moieties. The sample could be deformed and fixed when exposed to visible light with wavelength of $442 \mathrm{~nm}$. The authors observed that the fixed shape was stable for more than a year. Particularly intriguing was the fact that light at $442 \mathrm{~nm}$ induced repeated trans-cis and cis-trans photoisomerization, unlike typically used $365 \mathrm{~nm}$ light that would only cause the trans- to cis- isomerization. As the authors speculated, this repeated isomerization induced adjustments to the polymer chains in the glassy matrix, analogous to the typical thermal fixation. When the fixed sample was exposed to circularly polarized light of the same wavelength, photoreconfigured polymer network was randomized, leading to recovery.
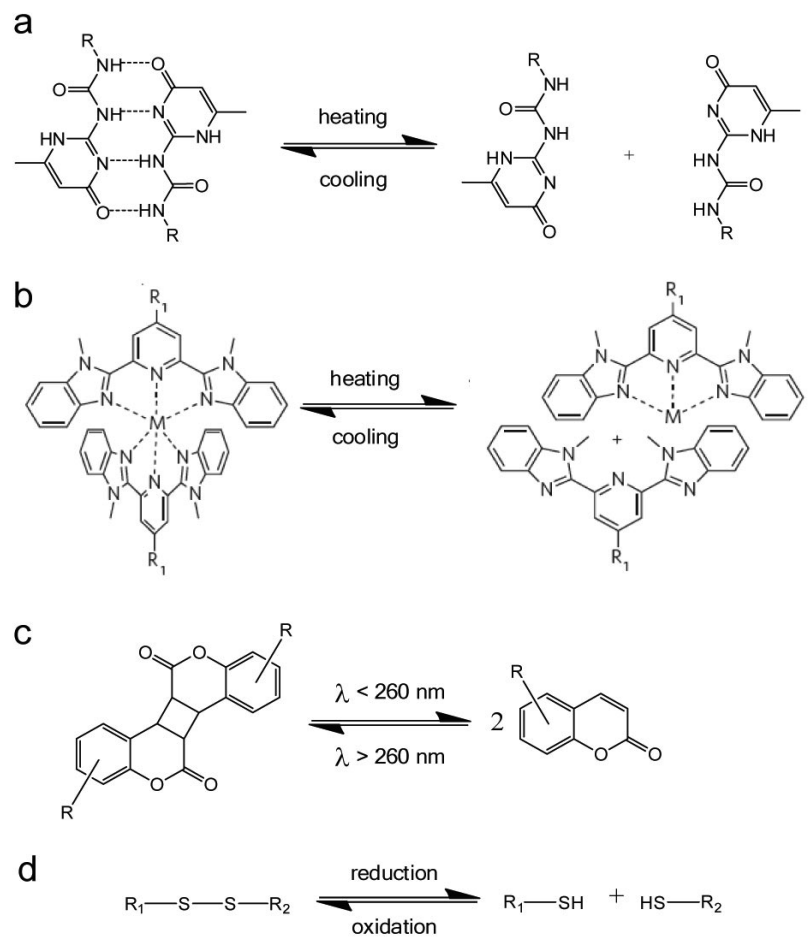

Fig. 6. Selective examples of molecular switches: (a). UPy coupling; (b). Metal-ligand coordination bonds [73], Copyright 2011. Reproduced with permission from Nature Publishing Group; (c). Coumarin [2+2] cycloaddition; (d). Disulfide bonds.

We note that light penetration deep into the SMP bulk is intrinsically difficult, limiting the effectiveness of light triggering to thin films and/or surfaces. On the positive side, many SMP applications that utilize only the surface shape memory effect have emerged recently [60-69]. It is thus foreseeable that the preceding SMP systems may find its niche due to the spatio-selectivity of the light triggering method.

Expanding the concept of molecular switches further, one can in principle design a variety of new SMPs by searching the library of stimuli-reversible bonds, selecting a desired one as the molecular switch and synthesizing a permanent network containing the reversible bonds. Possible molecular switches may include reversible covalent bonds (e.g., disulfide bonds, Diels-Alder adduct, and acylhydrazone bonds) and strong non-covalent physical interactions (e.g., multiple hydrogen bonding, metal-ligand coordination, and supramolecular recognition). Reviews on reversible chemical and physical bonds are particularly helpful [70-74]. It is 
important to note that the target network should possess sufficient chain mobility to facilitate the reversible chemical reactions (or physical interactions). Here, the reversible interactions simply form temporary crosslinking points while a set of permanent crosslinks should be in place to memorize the permanent shape. Selective molecular switches are presented in Fig. 6.

Several examples of SMP following the preceding design principle are presented in the following. A disulfide bond is a well known reversible covalent bond. To utilize such, thiol units were introduced as side groups onto cellulose acetate chains [75]. At the oxidized state, most of the thiol units tuned into disulfide crosslinks, enabling temporary shape fixing. Recovery was triggered by reducing the disulfide bonds into thiol units. Multiple fixing/recovery cycles could be realized. However, the SME decayed progressively as the result of redox side reactions. Moreover, the shape memory cycle time is very long (four days) due to the low reaction rate.

Strong physical interactions can also serve as the enabling molecular switches. For instance, ureidopyrimidinone (UPy) units form self-complementary hydrogen bonding (SCHB) interactions that are much stronger than typical hydrogen bonds [76-79]. The SCHB interactions possess a dynamic equilibrium feature that had been explored as molecular switches. In such an example, UPy units were introduced as the side groups onto a chemically crosslinked butylacrylate based elastomer network [77]. At a low temperature, two UPy units trended to strongly associate, establishing the temporary netpoints necessary to fix a deformed shape. Such a strong association corresponds to a very slow exchange between the associated UPy units. While at elevated temperatures, the equilibrium would shift towards the dissociated states (fast exchange mode), allowing recovery of the fixed shape. Perhaps due to the dynamic nature of the association, the deformation fixed by the SCHB at low temperatures would spontaneously recover by nearly $20 \%$ over one hour. Guan's group also reported a thermoplastic SMP containing cyclic UPy dimmers [80]. While the molecular design is quite different and in fact intriguing (i.e., the lack of permanent crosslinks), the material also shows a similar shape memory function and relaxation behavior. In contrast, the strain fixed by the reversible cinnamate cycloaddition exhibited hardly any relaxation over four hours in Lendlein's study [53]. As such, there are some small yet notable distinctions between these two types of molecular switches. For the dynamic UPy switches, the crosslinks would break and reform at any time (i.e., at equilibrium). An external stimulation only changes the kinetics. In contrast, the cinnamate molecular switches, while reversible, are non-dynamic (at least within the relevant timescale) and can only break or reform under two specific conditions (UV lights of different wavelengths). This comparison implies that, an SMP based on dynamic molecular switches may intrinsically exhibit the undesirable strain relaxation behavior; while such a behavior may not be expected for non-dynamic molecular switches. This particular point is somewhat speculative in nature and further studies are required to go deep into the operating mechanisms of reversible molecular switches.

In another study, Rowan's group synthesized a poly(butadiene) permanent network with metal-ligand coordination bonds as molecular switches [81]. Owing to the multi-responsiveness of the metal-ligand complex, shape recovery can be triggered by various methods including heating, UV irradiation, and specific chemical exposure that led to either 
decomplexation or increase the exchange kinetics between the metal ions and the ligands. Similar to the UPy based SMP, Rowan's SMP also showed 10 15\% strain relaxation one hour after the initial unloading. Again, this behavior is most likely due to the dynamic nature of the metal-chelating ligands.

The concept of molecular switches is not limited to the molecular interactions within the polymer matrix. A recent collaboration between Weder's group and Rowan's group led to a water triggered SMP composite system in which the molecular switching mechanism was the hydrogen bonding interactions between the polymer matrix and the nanocrystalline cellulose fillers $[82,83]$. In this case, water molecules diffused into the polymer-filler interface to dissociate the interfacial hydrogen bonding. At this state, a temporary shape was created and fixed by removal of water (thus reforming the interfacial hydrogen bonding) via drying. When the deformed sample was immersed in water, the interfacial hydrogen bonding was again dissociated, leading to the recovery. Here the water triggered recovery behavior is identical to Huang's system mentioned earlier, but the underlying mechanism is quite different. The former relies on water sensitive molecular switches whereas the latter operates by the water reduced phase transition temperature.

The preceding SMPs are all solids. The concept of molecular switches can be expanded to shape-memory hydrogels. In an aqueous environment, the choice of reversible interactions is even more readily available. However, we leave this discussion to a dedicated section on shape-memory hydrogels (Section 4).

In comparison to the phase switches, $R_{\mathrm{f}}$ of an SMP enabled by molecular switches is typically lower, at least for all the reported examples. From the molecular standpoint, a phase transition can freeze a very large portion (if not all) of the chain segments in a network, whereas molecular switches can only add a quite limited number of temporary netpoints to fix the deformed chains. A perhaps related macroscopic phenomenon refers to the elastic modulus change of an SMP between its activated state and the deactivated state. For a conventional $T_{\mathrm{g}}$ (or $T_{\mathrm{m}}$ )-type SMP, its elastic modulus at temperatures below its $T_{\text {trans }}$ (deactivated state) is usually $3 \sim 4$ orders larger than that at temperatures above its $T_{\text {trans }}$ (activated state). In contrast, typical molecular switches are only able to enlarge the modulus by at most 10 times due to the additionally formed crosslinking points. Despite the relatively low $R_{\mathrm{f}}$, molecular switches have greatly expanded the approaches for achieving alternative triggering mechanisms. This may be particularly helpful in the exploration of applications that require isothermal SMEs. Because of this unique advantage, one can envision that more examples of SMPs with novel and attractive triggering methods will emerge in the near future.

\subsection{Direct/indirect trigger methods}

Despite a few available molecular switches, most SMPs are based on reversible thermal phase transition that is intrinsically triggered by heating. The most common and convenient way to heat a material is directly increasing its surrounding temperature using hot gas or liquid (i.e., exogenous heating). However, such direct heating methods are not applicable in many cases encountered in applications. For example, when SMPs are used as morphing devices in aerospace applications, it is impossible to heat the devices by increasing the outer space 
temperature or blowing hot air onto the device. One solution to this is endogenous heating, namely, generating heat from inside the material. Electrical, magnetic, optical, acoustic and chemical energies can all be converted into thermal energy and cause heating within materials. The endogenous heating normally requires the incorporation of functional fillers into an SMP matrix to achieve the energy transformation. Conductive fillers such as carbon fibers, carbon nanotubes, carbon nanofibers, metal powders, etc. were incorporated into SMPs $[84,85]$. When electric currents pass through, these SMP composites can be triggered by Joules heating. Via induction heating, alternating magnetic fields can also induce internal heating within SMP composites containing magnetic nanoparticles $\left(\mathrm{Fe}_{2} \mathrm{O}_{3}, \mathrm{Fe}_{3} \mathrm{O}_{4}\right.$ or $\mathrm{Ni}$, etc.) [86-89]. The so-called photo-thermal effect has also been found effective by irradiating light of certain wavelength onto SMP composites containing carbon or gold nanofillers [90-94].

High intensity focused ultrasound (HIFU) was applied to remotely and locally heat SMPs [95]. HIFU, that can be concentrated onto a focal spot, was originally developed to treat tumors due to its thermal effect. The acoustic energy can convert into mechanical energy in the form of viscous shearing oscillation, that would subsequently be absorbed by an SMP in the form of temperature increases. Although the HIFU heating usually requires a liquid transfer media to ensure the generation of sufficient thermal energy, the ability to remotely heat a neat SMP is a unique advantage compared to the other indirect heating methods that can only heat SMP composites.

The concept of utilizing indirect heating has significantly expanded the scope of the recovery triggering methods. Unlike the non-thermal triggering methods enabled by the various molecular switches discussed in the last section, indirect heating rely on the same phase switches behind the conventional direct heating triggered SME. However, indirect heating triggers provide unparallel technological advantages due to their highly controllable nature (i.e., remote, local, and momentary). Electromagnetic wave, infrared light, and ultrasound can all penetrate through human skin. When an SMP is embedded into a human body as a biomedical device, the remote heating process can locally increase the temperature of the device. Due to the low heat transfer coefficient of polymers, it is possible not to spread too much of the heat into the surrounding body environment. Moreover, endogenous heating is usually much faster than exogenous heating due to its shorter heat diffusion length, important for polymers for which thermal conductivity is typically low. Of course, the ability to instantaneously turn the heating on/off in an on-demand fashion is also beneficial.

The endogenous heating could be coupled with exogenous heating to affect the apparent switching temperature $T_{\text {sw,app }}$ of an SMP [96],defined as the actual environmental temperature at which the recovery occurs. In this instance, due to the extra heat provided by the endogenous induction heating, the SMP can be triggered at an environment temperature that is lower than that without the endogenous heating. The extent of endogenous heat can be conveniently controlled by adjusting the endogenous heating power. As a result, $T_{\text {sw,app }}$ can be tuned without changing the actual material and its intrinsic $T_{\text {trans }}$.

Instead of heating, reducing $T_{\text {trans }}$ of an SMP to a temperature below the environmental temperature can also induce a recovery process. In 2005, Huang's group discovered that $T_{\mathrm{g}}$ of some commercial polyurethane SMPs could be greatly reduced using water as the plasticizer 
[52]. When the SMP was immersed in water, water molecules penetrated into the SMP bulk, broke the hydrogen bonding within the polyurethane segments, and consequently increased the chain mobility. As a result, $\mathrm{T}_{\mathrm{g}}$ of the SMP could be decreased below the ambient temperature, thus the recovery could be triggered without heating. In addition to polyurethane, SMP composites such as polylactide/cellulose composite [97] and polyvinyl alcohol/graphene oxide composite [98] were also reported to show water triggered SME. In both cases, water molecules destroyed the hydrogen bonding at the polymer-filler interfaces to trigger recovery. The water-induced SME can be also extended to $T_{\mathrm{m}}$-type SMP that contains hydrophilic crystalline poly(ethylene glycol) chains as the switches [99]. The shape recovery occurred when water molecules reduced the $T_{\mathrm{m}}$ of poly(ethylene glycol) to sub-ambient temperature. Although the water-induced SME achieved through reducing $\mathrm{T}_{\text {trans }}$ cannot be universally applied, it is particularly relevant for biomedical applications due to the natural presence of water in a human body and the difficulty to apply direct heating in those instances. An issue to consider here is that water induced recovery may take long times (e.g., days) due to the slow diffusion process. However, a recent effort by Mather's group led to an extremely fast (1.3 seconds) water responsive SMP by greatly reducing the diffusion length using microfibers to construct the SMP [100]. Another issue of the water-induced SME is that the shape recovery process would always be accompanied with a certain volume expansion of the SMPs due to the water absorption. This may or may not be an issue depending on the targeted application, but is a factor to keep in mind nevertheless.

Following the same principle of the water-induced SME, organic molecules such as DMF [101] and ethanol [102] can also be used to induce the SME. Unlike water naturally present in many environments SMP applications encounter, organic solvents do not naturally exist in mostly any cases. In fact, the use of organic solvents is quite undesirable for most applications. Thus, it is not clear what practical benefits such a triggering method may offer.

A particularly intriguing example is to use light to reduce the $T_{\text {trans }}$ of an SMP. In this example, spiropyran was doped into an ethylene-vinyl acetate copolymer (EVA) with a crystalline phase as the netpoints and a glass transition as the switching phase [103]. Upon UV irradiation at $365 \mathrm{~nm}$, spiropyran molecules isomerize to change from a closed spiro form to an open zwitterionic merocyanine form. The latter plasticized the amorphous phase to trigger the recovery. Thus, a UV-induced SME was realized. Such a mechanism is rather conducive to develop new SMEs.

\section{Variants in shape memory forms}

In the early days, SMPs were known to exist mostly in the form of dense bulk polymers. Increasingly, SMP in other forms were developed in efforts to meet various requirements in diverse potential applications. On this front, shape-memory surfaces and shape-memory microparticles that exhibit SMEs at the microscopic scales have attracted increasing attention. In addition, porous SMPs and shape-memory hydrogels have been developed to realize additional functions not offered by solid dense SMPs. The underlying molecular principle of the various forms of SMPs is identical to that of dense bulk SMPs. However, the diverse forms have greatly improved properties and extended the application potential for SMPs. For 
examples, a porous SMP allows extremely large volume changes relative to SMPs in its solid dense form. Since porous SMPs have been extensively reviewed in the literature, we hereafter concentrate on shape-memory surfaces, shape-memory hydrogels, and shape-memory microparticles.

\subsection{Shape memory surfaces}

Surface microstructures on polymers govern a variety of material properties such as adhesion, wetting, and friction, to name just a few. Consequently, fabricating and controlling microstructures on polymers are critical for numerous technologies. Extending the SME effect on the macroscopic bulk scale to microstructured surfaces thus offers great potential in terms of technological innovations. We note here that the molecular principle of shape memory surfaces is not different from bulk SMP. The mere difference lies in the form of deformation (surface versus bulk). However, as shall be illustrated below, this difference is non-trivial as it brings significantly more opportunities in terms of technological innovations. Overall, there are three scenario in which a surface SME is particularly relevant: 1) Creating erasable temporary surface microstructures; 2) Utilizing SMEs as a way for microfabrication of permanent surface microstructures; 3) Reversible tuning of permanent surface microstructures fabricated using conventional microfabrication methods.

Erasable temporary microstructures can be introduced on a featureless surface by micro-deformation. Nelson et al. [104] demonstrated an SME at a nanoscopic scale. In their work, nano-indents with depth less than $30 \mathrm{~nm}$ were created on a flat surface by pressing an AFM tip onto a thermoset SMP surface. The imprinted feature can fully recover upon heating. More important than the experimental demonstration itself is that, despite the microscopic heterogeneity inherent to a thermoset network, the SME appeared to remain valid at the nanoscopic scale. Whereas Nelson's work demonstrated the material capability, Altebaeumer et al. [105] extended this concept one step further. Relying on a massively parallel operation of scanning probes, thousands of nanoimprints were created onto a shape memory surface, this was viewed as a data writing process. These data (nanoimprints) can be erased and new data can be rewritten onto the surface by virtue of the SME. Intriguingly, the surface could sustain thousands of writing/erasing cycles without noticeable fatigue, implying the possibility of rewritable data storage. The surface SME is not just limited to the conventional dual-SMPs. Utilizing the multi-SME, discussed in more detail later in this review, three sets of micro-patterns could be imprinted onto a multi-SMP at three different temperatures [106]. Interestingly, the individual sets of surface patterns could be independently recovered at three recovery temperatures. This demonstration has the potential to greatly expand the scope of the surface SME.

SMPs have also found unique uses as a nonconventional microfabrication tool. An example of this is the use of an SMP to create surface nano-wrinkles. Surface wrinkles are often created using a rigid thin film (e.g., metal) supported on a soft thick substrate. Due to the modulus mismatch between the two layers, deformation to the substrate may lead to lateral compression to the rigid thin film. This would lead to mechanical instability and the formation of nanoscopic wrinkles. Typically a soft rubber is used as the wrinkle substrate. Because the 
deformation of rubber can only be conveniently created on a global level, it is difficult to realize spatio-control of wrinkle formation. In contrast, the surface deformation of an SMP can be easily controlled at any localized fashion via indentation. Following this principle, we demonstrated a new way to create spatially localized wrinkle (i.e., surface nano-structures) utilizing an SMP as the wrinkle substrate [60,61]. This principle can be potentially expanded to create other types of micro-structures and the methodology may open up new opportunities for microfabrication.

Surface microstructures fabricated on an SMP may be reversibly tuned after they are formed. Permanent microstructures can be directly fabricated onto an SMP surface using traditional microfabrication methods. Any deformation occurs at the macroscopic level, easy to introduce, translates directly into changes to the microstructure. An excellent example along this line is the use of an SMP to enable so-called programmable soft lithography [107]. In soft lithography, micro-molding is often time used. Typically, each elastomeric master mold can only be used to create one set of microstructures, the corresponding negative structures. However, when an SMP is used in place of the elastomer mold, multiple master molds may be created via macroscopic deformation of the original mold as schematically shown in Fig. 7a. This technique introduces flexibility for nanoimprint lithography or other micro-molding methods. Similarly, surface micro-features created by other methods such as a Breath Figure method can also be altered via the use of an SMP supporting substrate [108].

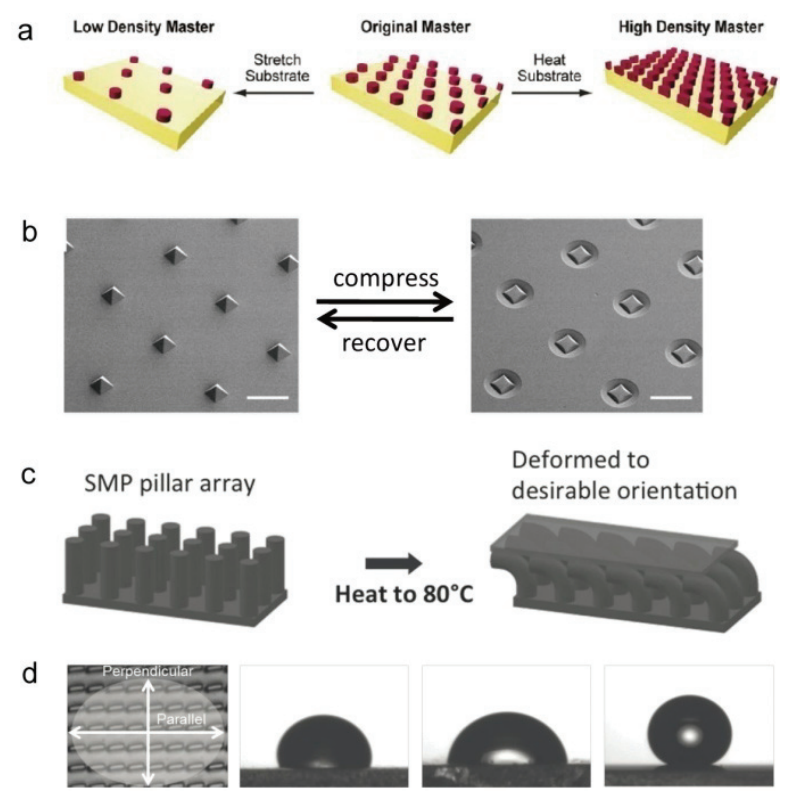

Fig. 7. Examples of shape memory surfaces: (a). Schematics of the programmable soft lithography [107], Copyright 2011. Reproduced with permission from the American Chemical Society; (b). SEM photos of the reversible dry adhesive (scale bar $=50 \mathrm{~mm}$ ) [68], Copyright 2013. Reproduced with permission from the American Chemical Society; (c). Schematics of the fabrication and deformation process of the shape memory pillar array; and (d). Anisotropic wetting on the deformed sample (c), from left to right: top-view of the deformed pillar array; water droplet viewed perpendicularly to the deformed pillars; droplet viewed in parallel to the deformed pillars; the droplet on the original surface [115], Copyright 2013. Reproduced with permission from John Wiley and Sons. 
Tuning surface microstructure via an SMP can also be utilized to fabricate multifunctional material systems (or devices). One strategy has been to couple surface dry adhesion property with an SMP to make reversible dry adhesives [62-68], e.g., molding micro-tips onto an SMP [68]. Despite the adhesion properties of the particular SMP used, the sharp micro-tips on its surface prohibit physical contact with potential bonding substrates. As a result, the micro-tipped SMP is in a non-adhesive state (Fig 7b, left). However, the surface micro-tips can be deformed via the SME, thus achieving conformal contact and bonding with the substrate (Fig 7b, right). Upon heating, the micro-tips returned to their protruded state, causing debonding. Such an SMP reversible adhesive possesses great potential for use in transfer printing, a key step in the manufacture of flexible electronics that is gaining increasing attention [109].

Pretsch et al. [110] demonstrated a concept using an SMP as a switchable information carrier. The surface of an SMP was first colored with a dye. Subsequently, the colored surface was patterned with machine-readable quick response $(\mathrm{QR})$ codes via laser ablation. Through shape programming, the tag prototype was deformed into temporary shapes with non-decodable QR code information. Upon heating, the original surface features were recovered and the $\mathrm{QR}$ code became readable again. The authors anticipated that tagging products based on this demonstration could be useful for one-time secure identification.

Cell growth is known to be sensitive to the topographic features of the substrate. Typically, only static surface topographic conditions can be realized. Recent work by several groups has demonstrated that, when a suitable SMP is applied as the cell culture substrate, the substrate can be programmed to change its topography under cell culture conditions. As such, different cell behavior can be observed in response to the surface micro-structure changing [111-114]. This ability to control the cell growth behavior via switchable surface topography adds a new "weapon to the arsenal".

Switchable surface topography enabled by an SMP can also be used to control surface wetting. Via replica molding, Yang's group fabricated high aspect ratio pillar arrays on an SMP surface (Fig. 7c) [115]. When the pillars were deformed into a bended state, water droplet on the surface exhibited a Wenzel state wetting with an anisotropic droplet shape, depending on the spacing between the pillars (Fig. 7d). Upon recovery of the pillars to the original straight state, the droplet displayed a Cassie-Baxter state wetting with a large contact angle and a small sliding angle. Such reconfigurable wettability may be potentially useful for water collection.

Micro-optics, that relies on surface microstructures to control the optical properties, plays an important role in modern technologies. Conventional micro-optics is fabricated on non-deformable materials such as glass. Once fabricated, the surface structure, thus the corresponding optical property, cannot be altered. In a recent collaboration the concept of shape memory surfaces was extended to a new generation of deformable and programmable micro-optics [69]. Via replica molding, the optically relevant surface microstructures were introduced onto an SMP. Macroscopic deformation via stretching or compression led to changes in the surface microstructure, thus the related optical properties (e.g., light 
transmission) (Fig. 8). This concept is not limited to any particular micro-optics, but can be extended to a variety of optical devices including retro-reflective surfaces, holograms, grating surfaces, and micro-lenses. In particular, the demonstration on holograms showed that the surface SME remains valid at a scale below $10 \mathrm{~nm}$.
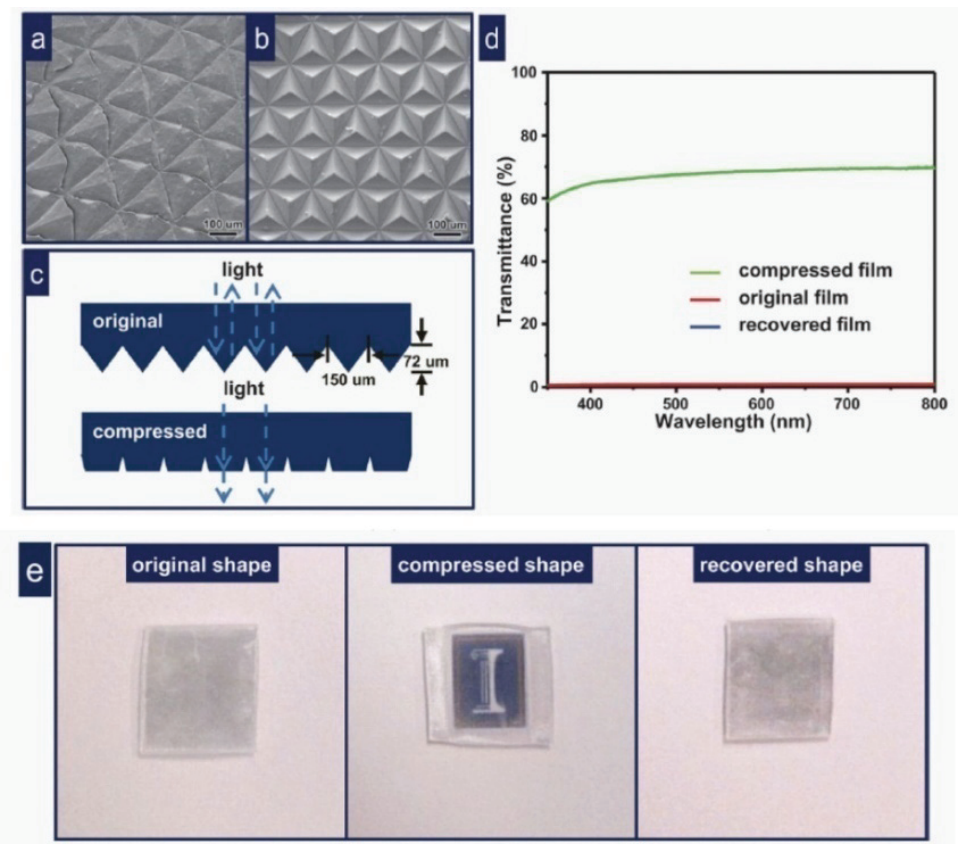

Fig. 8. Schematics of the programmable retro-reflective surface: SEM images of (a) Compressed (50\% strain) and (b) Recovered microprism array; (c). Schematic illustration of the total reflection effect of the microprism array; (d). Transmittance spectra of the shape memory film with microprism arrays under a shape memory cycle; (e). Optical photographs showing the optical response of (c) [69], Copyright 2013. Reproduced with permission from John Wiley and Sons.

Most of the concepts involving shape memory surfaces employ direct heating methods. In principle, all other forms of external stimulation methods can be readily applied [114,116]. Of particular interests are those that offer spatio-selectivity. They would permit valuable localized property manipulation, as can be imagined for the examples shown in the preceding. Whereas light stimulation is an obvious choice, an electronically programmed recovery is also an interesting alternative. This new form of stimulation relies on a micro-heater array installed on the backside of a shape memory surface [69]. The resistive heating through the micro-heater arrays can be controlled locally by electronic programs. Notably, the sequence of the spatio-recovery of the surface microstructures (and the associated functions) can be pre-defined by computer programs, a capability not offered by any other means of stimulation.

\subsection{Shape memory hydrogels}

Stimuli-sensitive hydrogels can change their shapes in response to external stimuli such as temperature, $\mathrm{pH}$, light, electric signal, or chemicals [2]. A typical stimuli-sensitive hydrogel is a crosslinked water-swollen network with environment sensitive groups. Usually, these groups can change their affinity with water rather than form additional crosslinking points upon 
stimulation. Thus, most stimuli-sensitive hydrogels show only non-programmable shape transformation between swollen states and shrunken states. Nevertheless, stimuli-sensitive hydrogels have been extensively studied for drug delivery, cell culture, fluid control, separation, and sensing applications not only because of their stimuli sensitivity, but also their unique softness, high hydrophilicity, biocompatibility, and ability for rapid diffusion of molecules $[117,118]$.

The programmable nature of shape memory hydrogels set them apart from typical stimuli-sensitive hydrogels. The SME of hydrogels was firstly demonstrated by Osada and coworkers in the mid-1990s $[119,120]$. A thermally triggered shape memory hydrogel was formed by water swelling of a polymer network obtained by copolymerizing of acrylic acid, stearyl acrylate, and methylenebisacrylamide (the crosslinker). Crystalline/melting transition of the stearyl side chains $\left(T_{\mathrm{m}}=50^{\circ} \mathrm{C}\right)$ serves as the switching segments and the crosslinker sets the permanent shape. As such, a temporary shape can be fixed upon deformation via cooling from above its melting temperature to room temperature. This, and other early examples of shape memory hydrogels rely on thermal reversible phase changes as the phase switches and their recovery is typically induced thermally [121-124]. As has been laid out in an earlier section, the concept of molecular switches has unparallel advantages in designing solid SMPs. The presence of water in hydrogels, on the other hand, creates even more opportunities for designing shape memory hydrogels using molecular switches. Theoretically, the same principle can be applied to organo-gels although not much has been reported in the literature. Examples of designing shape memory hydrogels using molecular switches are presented below.

Hydrophobic interactions, unique in an aqueous environment, have been demonstrated as a switch mechanism for shape memory. Inomata et al. [125] synthesized polypeptide hydrogel networks with hydrophobic alkyl side chains. At low molar fractions, the side chains form temperature dependent molecular segregations. A temporary shape was introduced at a high temperature associated with weakened segregation and fixed upon cooling due to the formation of stronger segregation. This temporary shape recovered gradually upon heating, due to the re-weakening of the segregation. In contrast, Huang and coworkers [126] developed an intriguing shape memory hybrid hydrogel that showed cooling-induced SME. Aqueous solution of poly(ethylene oxide)-poly(propylene oxide)-poly(ethylene oxide) [PEO-PPO-PEO] tri-block copolymer was incorporated into an elastic sponge to form the hybrid. Upon heating from room temperature to $35{ }^{\circ} \mathrm{C}$, the PEO-PPO-PEO solution resulted in a hydrogel due to hydrophobic interaction of the PPO block. In other words, heating led to additional temporary crosslinks that could fix the hybrid into a temporary shape. Shape recovery occurred when the PEO-PPO-PEO hydrogel melted upon cooling.

In addition to thermal stimulation, molecular switches have been increasingly applied to construct shape memory hydrogels that can be triggered by various other stimuli. Molecular switches made of metal ions-organics complexation can enable SMEs by controlling the oxidative state of the metal ions $[127,128]$. It was found that the complex formed between ferric ions $\left(\mathrm{Fe}^{3+}\right)$ and phosphate groups was effective in fixing a temporary shape (Fig. 9a) [127]. Upon reduction of $\mathrm{Fe}^{3+}$ to ferrous ions $\left(\mathrm{Fe}^{2+}\right)$, the original shape would be recovered due 
to the disassociation of the ion-phosphate physical crosslinking points. Similarly, the complex between copper ions and pyridine ligands was also applied as molecular switches to enable a redox-induced SME since temporary crosslinking complexes could be reversibly formed through controlling oxidative states of copper ions $\left(\mathrm{Cu}^{+}\right.$versus $\left.\mathrm{Cu}^{2+}\right)$ (Fig. 9b) [128]. Also of relevance is that the oxidative states of ions can be tuned both chemically and electrochemically.

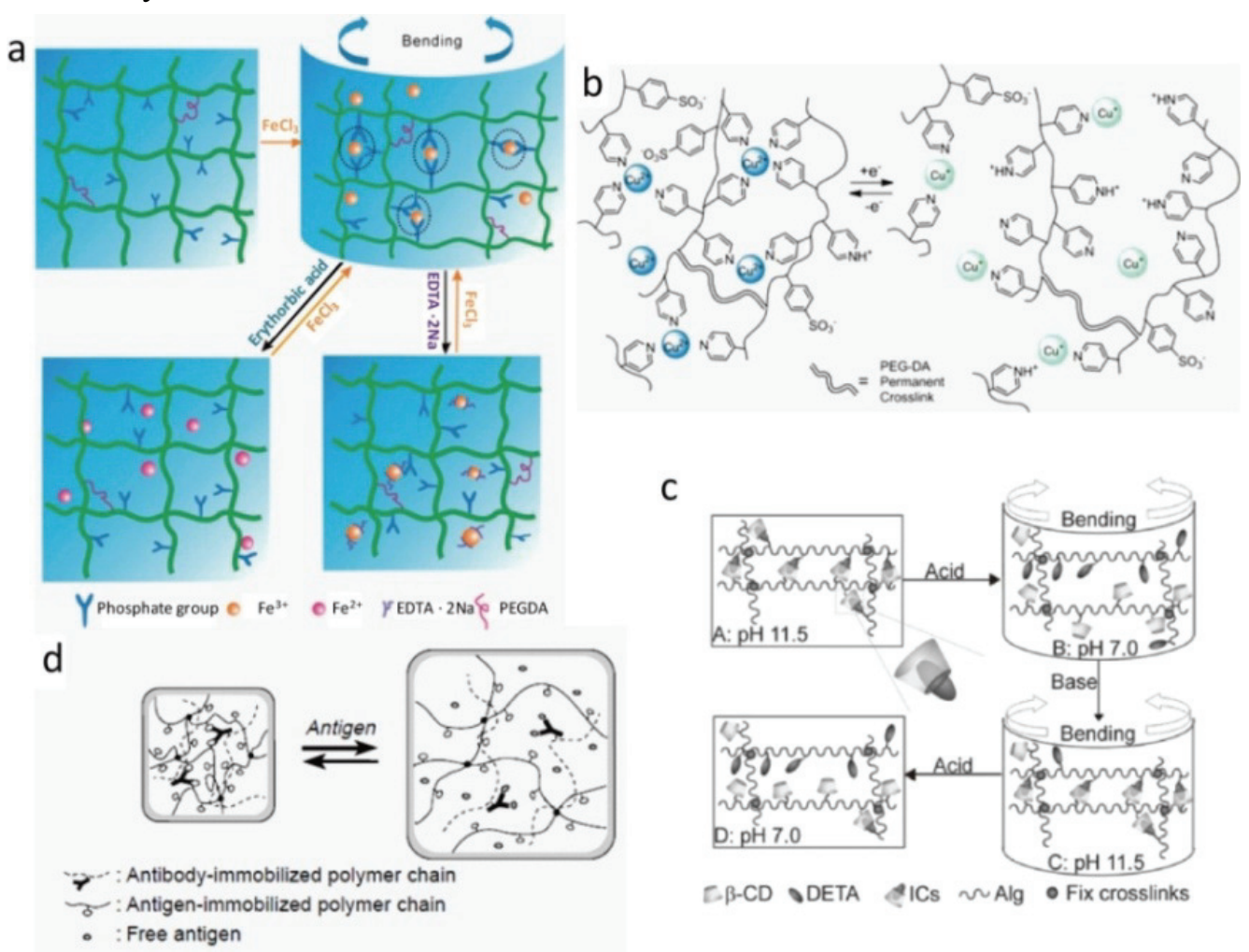

Fig. 9. Molecular mechanism of some shape memory hydrogels: (a). Iron ion-induced SME [127], Copyright 2014. Reproduced with permission from Royal Society of Chemistry; (b). Copper ion-induced SME [128], Copyright 2013. Reproduced with permission from the American Chemical Society; (c). pH-Induced SME [131],Copyright 2012. Reproduced with permission from John Wiley and Sons; and (d).Antigen-induced SME [135], Copyright 1999. Reproduced with permission from Nature Publishing Group.

Supramolecular physical linkage based on a host-guest complex is another catalog of molecular switches that has been applied to enable an SME in water swollen polymer systems. Cyclodextrins (CDs) are well known host compounds in supramolecular chemistry $[129,130]$. The molecular structure of CDs consists of a hydrophilic rim and a hydrophobic cavity. The cavity can capture a hydrophobic guest molecule with a proper size that is a little smaller than the cavity, forming a host-guest complex. When the hydrophobicity or size of the guest molecule is changed upon exposure to an external stimulus such as light, redox and $\mathrm{pH}$, the complexes would show a reversible assembly-disassembly behavior. An example using the host-guest complex as the molecular switches was recently reported by Zhang and coworkers [131]. They synthesized crosslinked alginate networks containing both $\beta$-CD and diethylenetriamine (DETA) as the side groups (Fig. 9c). In a basic medium ( $\mathrm{pH}=11.5)$, the DETA molecules showed a hydrophobic nature and the $\beta$-CD-DETA complexes were formed, 
that enabled the fixation of a temporary shape. While in a neutral medium, the DETA molecules became hydrophilic due to the partial ionization of the amine groups. In this case, the $\beta$-CD-DETA complexes disassociated, resulting in the macroscopic shape recovery. In a later study, the same group demonstrated that the redox-sensitive $\beta$-CD-ferrocene complexes could also be applied to enable an oxidation-induced SME due to the hydrophobic-hydrophilic transition occurred to the ferrocene groups at different oxidative states [132].

More intriguingly, the SME can be induced by glucose molecules if glucose oxidase was immobilized within the polymer network. In the presence of glucose, hydrogen peroxide was produced, that oxidized ferrocene. As a result, a glucose-induced SME was realized. It should be noted here that, the equilibrium water contents for the preceding systems were around $50 \%$, much lower than common hydrogels. Its versatility is quite notable nevertheless. Another report by Ritter and coworker [133] concentrated on a permanently crosslinked $\operatorname{poly}(N$-isopropylacrylamide) hydrogel with $\beta$-CD-adamantane temporary linkages. Adding a sufficient amount of free $\beta$-CD molecules to the system would disassociate the supramolecular temporary netpoints. Other permanently crosslinked hydrogels containing reversible supramolecular crosslinks (hydrogen bonds [134] or antibody-antigen interactions [135]) have been already developed. These hydrogels are intrinsically $\mathrm{pH}$ or antigen-induced (Fig. 9d) shape memory hydrogels. However, only non-programmable shape changing behavior (not the SME) had been investigated in these studies.

\subsection{Shape memory microparticles}

Multifunctional microparticles have been the subject of numerous studies due to their technological relevance in the biomedical field [136]. Despite their technological potential, shape changing microparicles had been limited to those exhibiting only non-programmable shape changing behavior $[137,138]$.

In 2014, Lendlein's group reported the first shape memory microparticle system $[139,140]$. In their work, microparticles with diameters around $\sim 30 \mu \mathrm{m}$ were prepared using an oil-in-water $(\mathrm{o} / \mathrm{w})$ emulsification method. The polymer used was a multi-block copolymer of poly( $\varepsilon$-caprolactone) [PCL] and poly $(\omega$-pentadecalactone) [PPDL]. Crystalline domains of PPDL segments $\left(T_{\mathrm{m}}=83{ }^{\circ} \mathrm{C}\right)$ and PCL segments $\left(T_{\mathrm{m}}=49{ }^{\circ} \mathrm{C}\right)$ served as the "permanent" netpoints and the switching segments, respectively. For programming, the microparticles in a permanent spherical shape were first embedded in a polyvinyl alcohol film $\left(T_{\mathrm{g}}=66^{\circ} \mathrm{C}, T_{\mathrm{m}}=\right.$ $163{ }^{\circ} \mathrm{C}$ ), followed by stretching the polyvinyl alcohol film at $70{ }^{\circ} \mathrm{C}$ and subsequent cooling. After dissolution of PVA in water, microparticles with the temporary ellipsoidal shapes were obtained. Shape recovery of the microparticles could be triggered upon heating. In the same work, the application of sonication enabled the preparation of SMP nanoparticles with much sizes around $250 \mathrm{~nm}$. Although crystallinity of the nanoparticles $(\approx 31 \%)$ was smaller than that of the microparticles $(\approx 39 \%)$, the SME was not noticeably affected since the minimum average crystallite size is approximately $16 \mathrm{~nm}$. Because the specific surface area of an ellipsoid was much larger than that of a sphere with the same volume, the authors envisioned that such SMP microparticles may provide switchable diffusion interfaces that could be 
potentially beneficial for controlled drug release.

\section{Multi-SMPs}

A dual-SMP can only switch between one temporary shape and its permanent shape in each shape memory cycle. In contrast, a multi-SMP can be broadly defined as a polymer that can be programmed to exhibit more than one distinctive shape change in the recovery event. This definition notably does not place any emphasis on fundamental enabling mechanisms. Instead, it focuses solely on the phenomelogical material behavior that is relevant for technological innovations.

A triple-SMP system (with two temporary shapes) was first reported by Lendlein's group in 2006 [141]. In the following 2-3 years, it was the only group working on this subject. For this reason, when they wrote a progress report on the subject in 2010, the focus was mostly on their own systems [142]. In 2011, at the time we published a review on the updated status of the general SMP field, the examples of multi-SMPs have bloomed to a point that we dedicated two sessions on the subject [4]. Few years have passed since then, most groups active in SMPs have now published their own multi-SMP systems. It is fair to say that the speed at which multi-SMPs have gained traction is not short of phenomenal [143]. We hereafter introduce the development in this very active research area.

As will be revealed in this section, the diverse strategies to design the broadly defined multi-SMPs are either strongly tied to the chemistry and physics intrinsic to the polymer matrix or extrinsic factors that are more independent of the polymer matrix (e.g., innovative actuation methods). To emphasize this mechanistic difference, we classify multi-SMPs into two categories: intrinsic multi-SMPs and extrinsic multi-SMPs.

\subsection{Intrinsic multi-SMPs}

\subsubsection{General aspects}

A multi-SMP is not fundamentally different from a dual-SMP. In fact, the molecular requirements for intrinsic multi-SMPs are quite similar to that of dual-SMPs. The network structure that is responsible for memorizing the permanent shape for a dual-SMP should also be present in a multi-SMP. The difference is in the switches, that should be more versatile for a multi-SMP. A typical triple-SMP should possess two thermal transitions that can be utilized to fix two independent temporary shapes. In addition, the realization of the triple-SME typically requires a two-step programming instead of one-step for the dual-SME. This two-step programming was called a triple-shape-memory creation process by Lendlein's group. Without the two-step programming, an SMP system with two $T_{\text {trans }}$ s would not exhibit the triple-SME [144]. It should also be noted that the multiple temporary shape recoveries differ from intermediate shapes occurring along a single recovery pathway for a dual-SMP. Although intermediate shapes can be obtained via on and off application of the stimulus, they are not independent to each other. Figure 10 illustrates the recovery of a triple-SMP [145]. By comparing the three shapes in Fig. 10, it is quite obvious that the temporary shape B definitely altered the recovery pathway from the temporary shape $A$ to the permanent shape $C$, in a discontinuous fashion. Intermediate shapes, by comparison, can only be found along the same 
recovery pathway. Thus, triple-SMPs provide much more controllable and complex shape morphing ability.
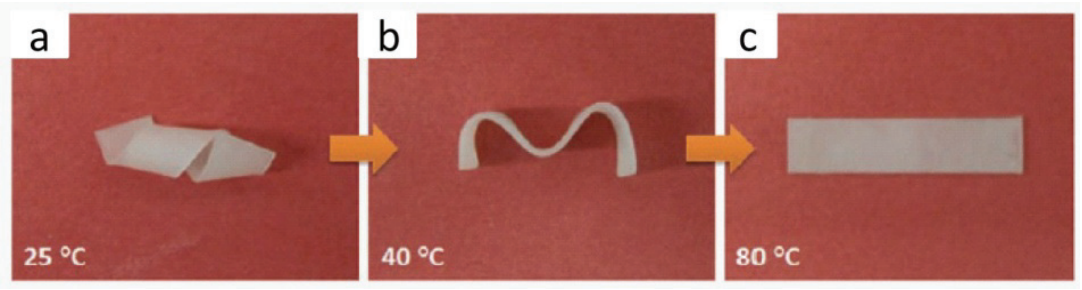

Fig. 10. Photographs showing the sequential recovery of a triple-SMP from temporary shape C (a), to temporary shape B (b), and to permanent shape A (c) [145], Copyright 2010. Reproduced with permission from John Wiley and Sons.

A typical quantitative triple-shape cycle is presented in Fig. 11b (146). In comparison to a dual-shape cycle with a single fixing and a single recovery steps (Fig. 4a), the triple-shape cycle contains two programming (shape fixing) steps and two recovery steps. The polymer has two thermal transition temperatures, $T_{\text {trans,B }}$ and $T_{\text {trans,A. }}$. At the programming stage, the permanent shape $\mathbf{A}\left(\varepsilon_{\mathrm{A}}\right)$ is first deformed to $\varepsilon_{\text {Bload }}$ at $T_{\text {high }}$ (above both $\mathrm{T}_{\text {trans }} \mathrm{s}$ ) and then cooled to $T_{\text {mid }}\left(T_{\text {trans, } \mathrm{B}}<T_{\text {mid }}<T_{\text {trans, } \mathrm{A}}\right)$, resulting in the temporary shape $\mathbf{B}\left(\varepsilon_{\mathrm{B}}\right)$ after releasing the stress. In the second programming step, the shape $\mathrm{B}$ is further deformed to $\varepsilon_{\text {Cload. }}$. A second temporary shape $\mathbf{C}\left(\varepsilon_{\mathrm{C}}\right)$ can be fixed upon cooling to $T_{\text {low }}<T_{\text {trans, }}$ and subsequent removal of the second stress. The shape recovery is typically conducted under a stress free condition. Heating shape $\mathbf{C}$ from $T_{\text {low }}$ to $T_{\text {mid }}$ leads to the recovery of the shape $\mathbf{B}\left(\varepsilon_{\text {Brec }}\right)$ while further heating to $T_{\text {high }}$ results in the recovered shape $\mathbf{A}\left(\varepsilon_{\mathrm{Arec}}\right)$. The respective shape stability $\left(R_{\mathrm{f}}\right)$ and shape recovery $\left(R_{\mathrm{r}}\right)$ can be calculated using the following equations and strain data obtained from the triple-shape cycle:

$$
\begin{aligned}
& R_{\mathrm{f}}(\mathrm{X} \rightarrow \mathrm{Y})=\left(\varepsilon_{\mathrm{Y}}-\varepsilon_{\mathrm{X}}\right) /\left(\varepsilon_{\mathrm{Yload}}-\varepsilon_{\mathrm{X}}\right) \\
& R_{\mathrm{r}}(\mathrm{Y} \rightarrow \mathrm{X})=\left(\varepsilon_{\mathrm{Y}}-\varepsilon_{\mathrm{Xrec}}\right) /\left(\varepsilon_{\mathrm{Y}}-\varepsilon_{\mathrm{X}}\right)
\end{aligned}
$$

where $\mathrm{X}$ and $\mathrm{Y}$ denote two different shapes, respectively.

a

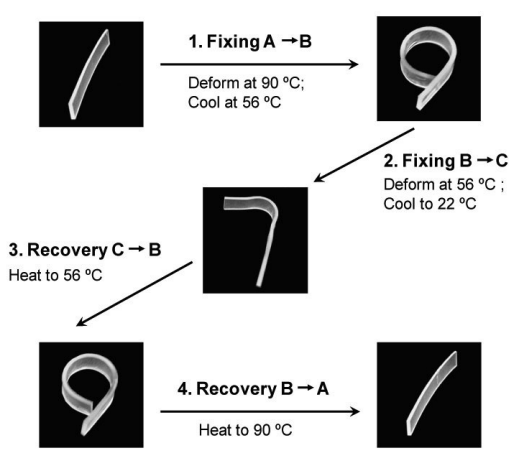

$\mathrm{b}$

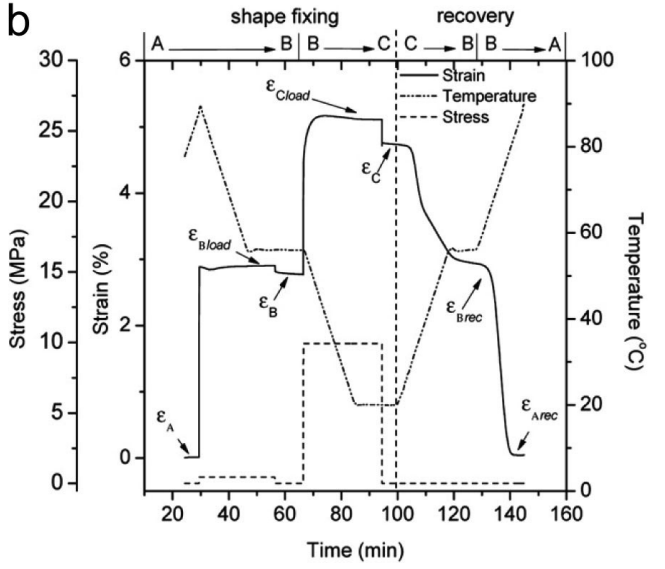

Fig. 11. Triple-shape memory cycles: (a). Visual demonstration; and (b). Quantitative themomechanical cycle [146], Copyright 2009. Reproduced with permission from John Wiley and Sons. 
The preceding triple-shape cycle is obtained under a stress controlled programming condition and a stress free recovery condition. Alternatively, the programming can also be conducted under a strain-controlled mode, while the recovery can be performed under iso-strain condition. This situation is quite similar to a dual-shape cycle introduced in section 2. In addition to the commonly used two-step programming procedure (2SPP), a one-step programming procedure (1SPP) may also be employed. The 1SPP can be conducted at either $T_{\text {high }}$ or $T_{\text {low }}$ (i.e., cold drawing). A two-step recovery can still be observed during reheating despite the one-step fixation. The 1SPP technique represents a simpler way to for achieve a triple-SME. However, the technique cannot program two independent strains into the SMP. Therefore, the 2SPP is still the preferred method for creating complex shape transformations.

From the preceding description, it can be seen that the triple-SME is a natural extension of the dual-SME. Likewise, triple-SME can be further expanded to quadruple-SME (and beyond) following the same principle. From a practical sense, multi-SMPs should provide properties and functions that can be tuned to meet the requirements for potential applications, similar to dual-SMPs discussed in Section 2.4. To this end, various strategies to design multi-SMPs have been reported in the literature, as discussed in the following.

\subsubsection{Multi-SMPs via distinct thermal phase switches}

In its simplest form, a triple-SMP should have two distinct thermal transitions and one set of netpoints. Copolymerization is the most natural choice for introducing multiple thermal transitions. The first two triple-SMP systems were both covalently crosslinked networks each of which has two switch phases [141]. The first example was obtained by UV curing of cyclohexyl methacrylate (CHMA) monomer and poly(caprolactone)dimethacrylate [PCLdimethacrylate] crosslinker. This yielded a permanent network with two thermal transitions from the crystalline PCL with $T_{\mathrm{m}}=50^{\circ} \mathrm{C}$ and glassy poly(cyclohexyl methacrylate) [PCHMA] with $T_{\mathrm{g}}=140{ }^{\circ} \mathrm{C}$ (Fig. 12a). The second triple-SMP network was synthesized similarly except that the CHMA monomer was replaced by a polyethylene glycol (PEG) monoacrylate, this yielded a crosslinked PCL $\left(T_{\mathrm{m}}=50{ }^{\circ} \mathrm{C}\right)$ network with PEG $\left(T_{\mathrm{m}}=34{ }^{\circ} \mathrm{C}\right)$ grafted chains (Fig. 12b). Despite the difference in the network architecture and the nature of thermal switches (one amorphous plus one crystalline versus two crystalline phases), both networks possessed two distinct thermal transitions and showed a triple-SME. In fact, ample examples showed below suggest that combination of any two thermal reversible phase transitions (glass transition, melting transition, or liquid crystalline transition) would enable a triple-SMP.

In 2009, Mather's group reported a liquid crystalline homopolymer (a polyester) with a glass transition $\left(80^{\circ} \mathrm{C}\right)$ and isotropic-nematic transition $\left(150^{\circ} \mathrm{C}\right)[147]$. The polymer exhibited two recovery events that are characteristic of a triple-SMP, although the authors did not call this a triple-SME. Because both the transitions are linked to the same monomer, this homopolymerization approach does not allow easy independent tuning of two transition phases. Ahn and coworkers $[148,149]$, on the other hand, synthesized a crosslinked glassy network 
containing liquid crystalline side chains via ring-opening methathesis polymerization (ROMP) followed by radical initiated thermal crosslinking (Fig. 12c). The polymer also exhibited triple-shape functions. Unlike the preceding case, however, the $T_{\mathrm{g}}$ and the $T_{\mathrm{cl}}$ of this system correspond to the main network and the side chains, respectively. This should allow independent tuning of the two responsible thermal phase switches, similar to what was possible with Lendlein's copolymerization approach.

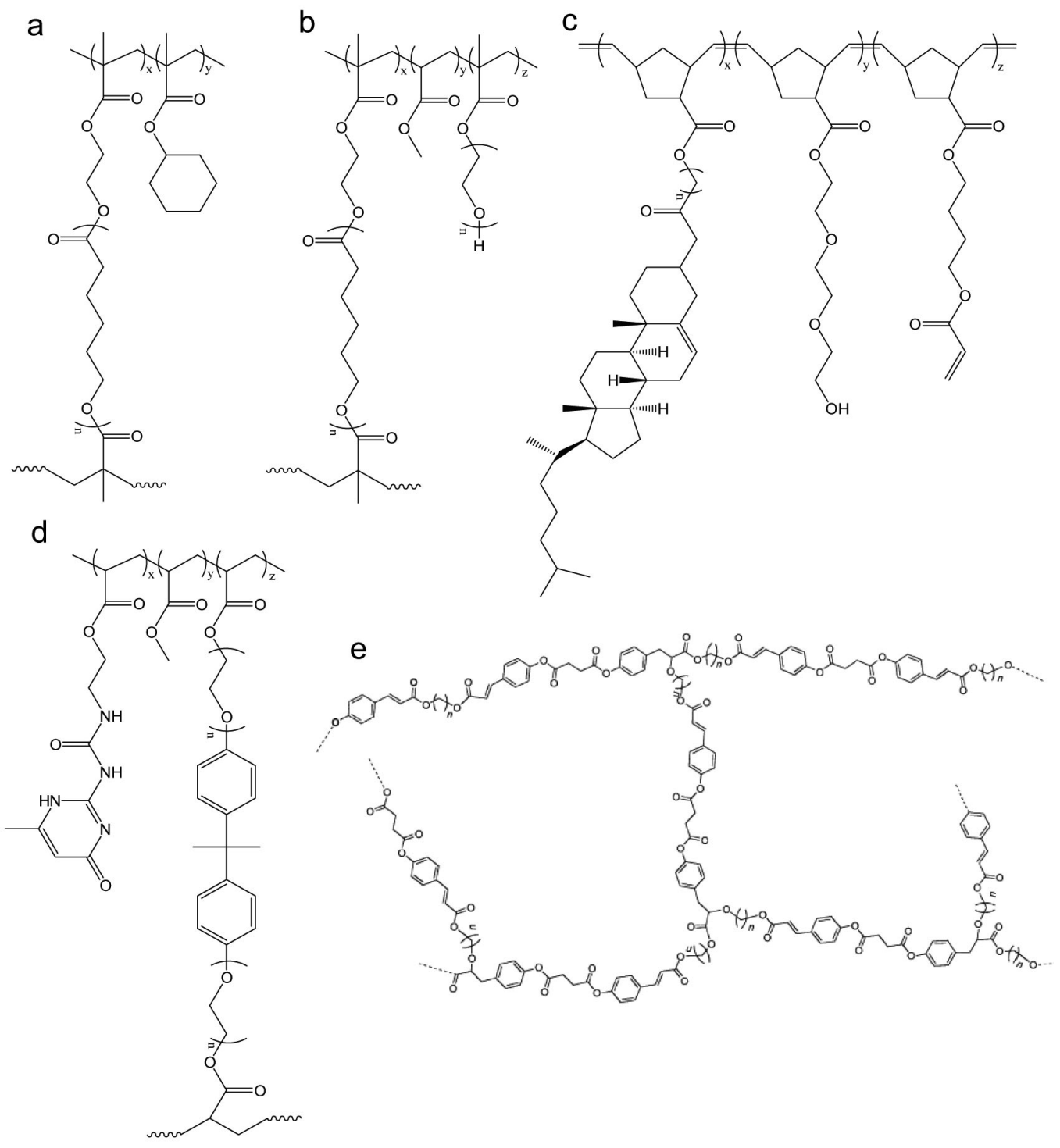

Fig. 12. Molecular structures of selective triple-SMPs [158], Copyright 2013. Reproduced with permission from John Wiley and Sons.

A "grafting to" technique is another way to design a triple-SMP. Polybutylene succinate (PBS) was grafted onto maleated-polystyrene-b-poly(ethylene-co-butylene)-b-polystyrene (m-SEBS) [150]. The poly(ethylene-co-butylene) segments within the m-SEBS backbone and the grafted PBS segments provided two $T_{\mathrm{m}} \mathrm{s}\left(60^{\circ} \mathrm{C}\right.$ and $110^{\circ} \mathrm{C}$, respectively), whereas the high glass transition of the amorphous polystyrene phase provided the physical netpoints. Notably, since there was no permanent crosslinking, the triple-SMP system could be reprocessed.

A triple-SMP can also be designed via an interpenetrating polymer network (IPN) 
approach [151]. UPy end-capped star-shaped PCL and hydroxyl groups terminated poly( $p$-dioxanone) [PPDO] were used. Crosslinking PDDO via its reaction with a diisocyanate led to a PPDO network while the PCL naturally formed a supramolecular network via the self-complementary hydrogen bonding between UPy units. The two crystalline phases $\left(T_{\mathrm{m}} \mathrm{s}\right.$ of $40{ }^{\circ} \mathrm{C}$ and $95^{\circ} \mathrm{C}$, respectively) provided by PCL network and PPDO network formed the basis for the triple-SME. They further demonstrated that the ratio between the PCL and PPDO within the IPN could be easily tuned without affecting much the two transition temperatures. One interesting benefit of the IPN design was that the crystallizability of both the PCL and PPDO segments was much better than that in a co-network.

Blending two (or more) polymers with different transition temperatures followed by crosslinking is a versatile approach to prepare a multi-SMP [152,153]. For instance, a linear high density polyethylene $\left(T_{\mathrm{m}}=132{ }^{\circ} \mathrm{C}\right)$ and two ethylene-1-octene copolymers with $T_{\mathrm{m}} \mathrm{s}$ of $59{ }^{\circ} \mathrm{C}$ and $96^{\circ} \mathrm{C}$ were blended and then thermally crosslinked with a peroxide. This yielded a crosslinked network with three distinct melting events, resulting in a quadruple-SMP. Choosing various other polyolefins with different $T_{\mathrm{m}} \mathrm{s}$ would allow tuning the multi-SMP.

Doping small molecules into a dual-SMP network represents another interesting approach to introduce an additional transition phase [154,155]. In one example, a thermoplastic polyurethane was synthesized by polymerization of PCL-diol, 4,4'-methylenediphenyl diisocyanate (MDI), pentaerythritol, and 2,2-bis(hydroxymethyl) propionic acid [154]. The PCL and the glassy hard domains provided a $T_{\mathrm{m}}$ around $50{ }^{\circ} \mathrm{C}$ and a $T_{\mathrm{g}}$ around $70{ }^{\circ} \mathrm{C}$. More importantly, the presence of carboxylic acid groups provided a mechanism for further doping. Thus, liquid crystalline small molecules, cholesteryl isonicotinate (INCh), were doped into the polyurethane via the ionic hydrogen bonding between pyridine on INCh and carboxylic acid groups. With a high transition temperature (above $110^{\circ} \mathrm{C}$ ), INCh formed the basis for physical netpoints. Thus, a thermoplastic triple-SMP was obtained. Similarly, Chen et al. [155] doped 4-hexadecyloxybenzoic acid (HOBA) into a thermoplastic dual-shape memory polyurethane (SMPU) with a $T_{\mathrm{g}}$ of $90^{\circ} \mathrm{C}$. HOBA is a liquid crystal mesogen with a transition temperature of $100{ }^{\circ} \mathrm{C}$. Within the network, it also acted as a plasticizer, lowing the $T_{\mathrm{g}}$ of the SMPU to around $50{ }^{\circ} \mathrm{C}$, while not destroying the H-bonding crosslinks inherent to the SMPU network. The liquid crystal doped SMPU showed not only a triple-shape effect due to the two thermal transitions, but also a nematic phase upon heated to a temperature between $100{ }^{\circ} \mathrm{C}$ and $130{ }^{\circ} \mathrm{C}$.

The intrinsic multi-material nature of composites naturally points to a highly attractive and versatile option to create multi-shape functions. In fact, one of the first few examples of a triple-SMP was a composite laminate consisting of two layers of thermoset epoxy polymers with well-separated $T_{\mathrm{g}} \mathrm{s}$ [146]. Although the composite laminate was macroscopically heterogeneous, it met the basic structural requirement for a triple-SMP. When the interface between the layers was sufficiently strong, the composite readily exhibited the triple-SME. Similarly, a triple-SMP composite was prepared by incorporating two shape-memory polyurethane layers that were permanently crosslinked by silica nanoparticles [156]. Mather's group pursued a different approach to create a triple-SMP composite [145]. They fabricated non-woven thermoplastic PCL nanofibers $\left(T_{\mathrm{m}}\right.$ around $\left.55^{\circ} \mathrm{C}\right)$ via electrospinning. The fiber 
mats were then embedded into an epoxy dual-SMP matrix with $T_{\mathrm{g}}$ around $35{ }^{\circ} \mathrm{C}$. The composite also displayed good triple-SME. One common characteristic for the three composite examples shown here was that the individual contributing components could be independently adjusted both in terms of transition temperatures and their fractions. This is quite advantageous in tuning triple-shape functions.

\subsubsection{Multi-SMPs via molecular switches}

Section 3.1 described molecular switches for dual-SMPs. We show in this section that various molecular switching mechanisms can also be utilized to enable triple-SMPs, either by themselves or in combination with a thermal phase switch.

We described earlier in this review that supramolecular self-complimentary hydrogen bonding by UPy is an effective molecular switch for elastomeric dual-SMPs. Voit's group synthesized a UPy containing chemically crosslinked (meth)acrylate network with a $T_{\mathrm{g}}$ tunable between 0 and $60{ }^{\circ} \mathrm{C}$ (Fig. 12d) [157]. When the $T_{\mathrm{g}}$ does not overlap with the UPy thermal activation temperature, they found that both the amorphous phase and the supramolecular molecular switch could be used to record two independent shapes, thus achieving a triple-SME.

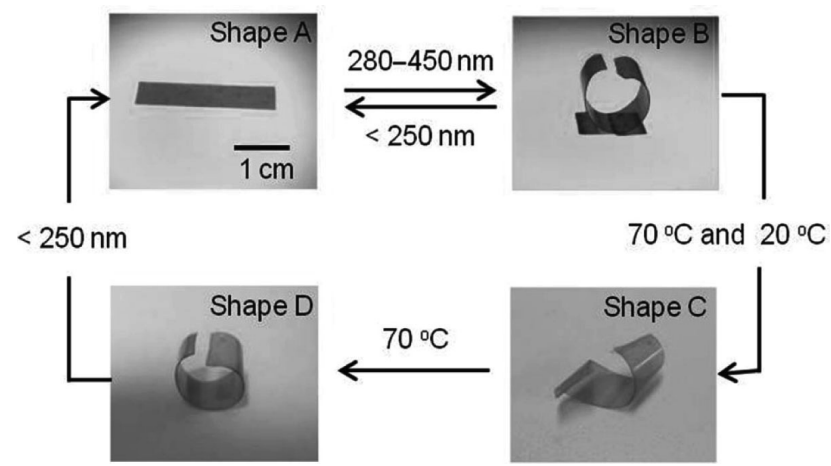

Fig. 13. Series of photographs of the hyperbranched coumarate polyester sample showing a photo-induced mutlti-SME. The permanent shape A was irradiated by UV light at $\lambda=280-450 \mathrm{~nm}$ for $20 \mathrm{~s}$ to form shape $\mathbf{B}$. Upon being deformed by an external load to the screw shape at $70{ }^{\circ} \mathrm{C}$ and cooled/fixed at room temperature, shape $\mathbf{C}$ was fixed. Shape $\mathbf{D}$, the same as shape $\mathbf{B}$, was recovered by heating again to $70^{\circ} \mathbf{C}$. Shape $\mathbf{A}$ was recovered by irradiation at $\lambda=250 \mathrm{~nm}$ for 180 minutes. The size of shape $\mathbf{A}$ is 25 $\mathrm{mm} \times 10 \mathrm{~mm} \times 0.2 \mathrm{~mm}$ [158], Copyright 2013. Reproduced with permission from John Wiley and Sons.

A photo-responsive molecular switch can also be incorporated into a polymer with a thermal phase switch to enable a multi-SME [158]. The polymer showing this feature is a hyperbranched coumarate polyester, synthesized by the polycondensation between a glycol and a diester monomer containing cinnamic double bonds (Fig. 12e). With its Tg between 36 and $64{ }^{\circ} \mathrm{C}$, the polymer showed a thermally induced dual-SME. In addition, reversible photo-deformation capability was also observed. Upon UV $(\lambda=280 \sim 450 \mathrm{~nm})$ light exposure to the polymer film from the upper side, it transformed to a concave shape (Fig. 13) due to the uneven formation of cyclobutane crosslinks through the film thickness. The cyclobutane 
structure was stable after the UV light was turned off, so that the concave shape could act as the permanent shape for a thermally induced SME (Fig. 13). When needed, the concave shape could be erased upon UV exposure of $\lambda=250 \mathrm{~nm}$. In short, the hyperbranched polyester provided photo and thermal switching mechanisms that contribute to its higher versatile multi-SME. Notably, the photo-induced deformation could be sorted as a special SME in which the corresponding programming was achieved by the internal stress generated via the uneven light exposure, in a hands free fashion.

A water sensitive molecular switch was also used in combination with a thermal phase switch to design a triple-SMP. To this end, Hu's group fabricated a triple-SMP nanocomposite using cellulose-whiskers as reinforcement and a segmented polyurethane SMP as the matrix [159]. In addition to the thermal phase switch inherent in the polymer matrix, the hydrogen bonding within the percolation network of cellulose-whiskers formed an additional set of water sensitive switch. The composite deformed in warm water can be fixed via cooling and drying to yield a temporary shape $\mathbf{A}$. Via heating under a dry condition, shape $\mathbf{A}$ recovered to a shape B. By immersing in warm water, the shape $\mathbf{B}$ recovered further to the original shape $\mathbf{C}$.

A single set of molecular switches exhibiting two different molecular states represents a highly intriguing mechanism to achieve a non-thermal triple-SME. Liu's group synthesized crosslinked hydrogels by copolymerization of acrylonitrile and 2-methacryloyloxyethyl phosphorylcholine [160]. In pure water and a low concentration of $\mathrm{ZnCl}_{2}$ solution, the cyano groups formed strong $\mathrm{CN}-\mathrm{CN}$ dipole-dipole interactions and $\mathrm{Zn}(\mathrm{CN})_{2}$ complex, respectively. When the $\mathrm{ZnCl}_{2}$ concentration was increased to a high level (> 38\%), each individual cyano group would associate with one zinc ion, thus destroying both interactions. At this latter state, the hydrogel possessed the permanent shape. Upon subsequent immersion in pure water, a deformed temporary shape could be fixed due to the formation of strong dipole-dipole interactions that acted as the temporary crosslinks. A second type of additional crosslinks formed when the hydrogel was soaked in a relatively low concentration $(<30 \%) \mathrm{ZnCl}_{2}$ solution, due to the formation of $\mathrm{Zn}(\mathrm{CN})_{2}$ complexes. These two types of molecular switches were utilized to independently fix two temporary shapes and subsequently recover when the solution conditions were reversed. That is, the hydrogel displayed a triple-SME (Fig. 14a). In addition to the triple-SME, the dipole-dipole interactions and the $\mathrm{Zn}(\mathrm{CN})_{2}$ complex are quite beneficial for the mechanical properties of the hydrogel. At an equilibrium water contents higher than $75 \%$, the hydrogel possessed tensile strength ranging from $1.5 \mathrm{MPa}$ to $4.3 \mathrm{MPa}$ and high Young's modulus up to $39 \mathrm{MPa}$. An additional feature is that increasing/decreasing additional crosslinks also impacted the volume of the hydrogel due to the change in the equilibrium water content. That is, the hydrogel displayed both the SME and a non-programmable shape change at the same time. In this case, it is more appropriate to evaluate its shape memory properties such as $R_{\mathrm{f}}$ and $R_{\mathrm{r}}$ via bending tests instead of tensile tests, since the bending angles are less impacted by the swelling/shrinking of the material. The combination of non-programmable shape changing behavior and the SME could be used to achieve very sophisticated shape-morphing behavior. Indeed, when the hydrogel was patterned with stripes on one side of the surface, by controlling the directions of the stripes, various curling shapes were realized upon exposure to zinc ions [161]. This is shown in Fig. 14b. The 
curling was attributed to heterogeneous volume change within the hydrogel. Due to its larger surface area, the volume change on the surface-patterned side was faster than the non-patterned side. In addition to its intriguing shape memory behavior, the hydrogel possessed low cytotoxicity and might be potentially useful as a tissue engineering scaffold [162].

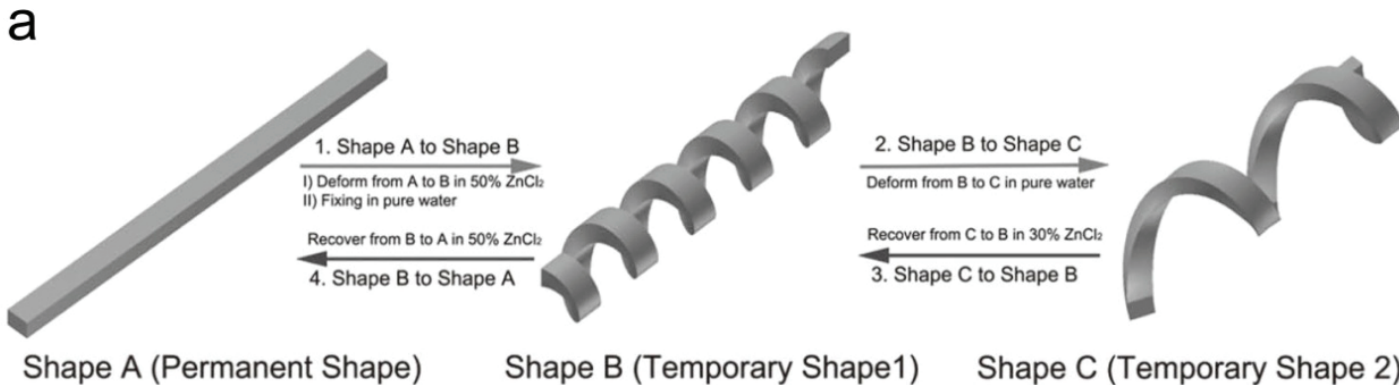

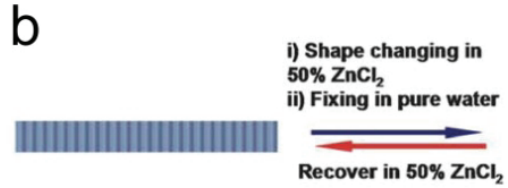

PAS-15-5-0

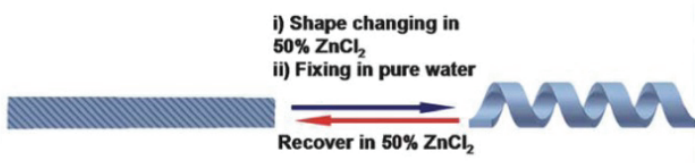

PAS-15-5-45

Fig. 14. Zn ion induced shape memory effect in hydrogels. (a) Schematic illustration of the triple-SME [160], Copyright 2012. Reproduced with permission from John Wiley and Sons. (b) A schematic diagram illustrating automatic shape changing of the hydrogel with surface patterned stripes of different angles [161], Copyright 2012. Reproduced with permission from Royal Society of Chemistry.

\subsubsection{Tunable multi-SMPs via a single broad thermal phase switch}

The extension from a dual-SME to a triple-SME seemed to imply that the number of temporary shapes that a polymer can fix is determined by the number of distinct phase transitions. Thus, any additional distinct phase transitions would theoretically bring in additional temporary shapes. However, synthesizing a polymer network with multiple $(>2)$ discrete thermal transitions, while possible, becomes increasingly more challenging as the number of transitions goes up. In 2010, we discovered a rather different approach to achieve a multi-SME [163].

Nafion, a thermoplastic perfluorosulfonic acid ionomer (Fig. 15a), has a single broad thermal phase transition ranging from $55^{\circ} \mathrm{C}$ to $130{ }^{\circ} \mathrm{C}$ (Fig. 15b). We discovered that, within this single phase transition, one could program two temporary shapes into the material at two deformation temperatures $\left(T_{\mathrm{d}} \mathrm{s}\right)$. Subsequent heating (stress free) to the corresponding recovery temperatures (with $T_{\mathrm{r}}=T_{\mathrm{d}}$ ) led to two distinct recovery events. In essence, a triple-SME was realized without the need for two discrete transitions. In addition, a quadruple-SME (possibly beyond) could be realized with this single transition (Fig. 15c). This type of multi-SME is different from a triple-SMP with two distinct transitions. For the latter, the two recovery events always occur at two temperatures in between and above both transition temperatures, respectively. Thus, if one wishes to move the recovery temperatures, as may be demanded for different applications, the transition temperatures will have to be altered. This would require 
synthesis of a new polymer. For Nafion, however, the multiple temporary shapes would recover fully at the recovery temperatures corresponding to its deformation temperatures $\left(T_{\mathrm{r}}=\right.$ $T_{\mathrm{d}}$ ). Therefore, the recovery temperatures for the temporary shapes could be altered via the choice of $T_{d}$, instead of having to synthesizing new polymers. We call this behavior the tunable multi-SME, emphasizing that the recovery temperature can be physically tuned (or manipulated) within one polymer.

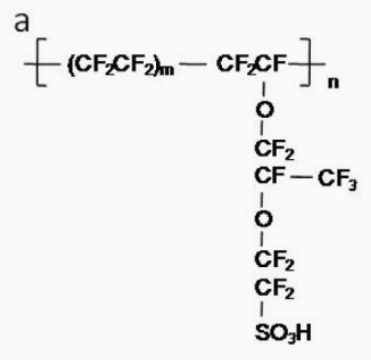

C

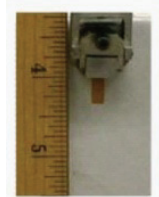

so

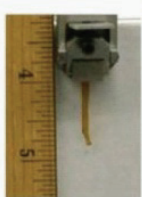

s1

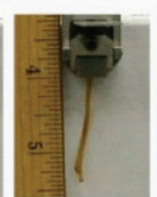

S2

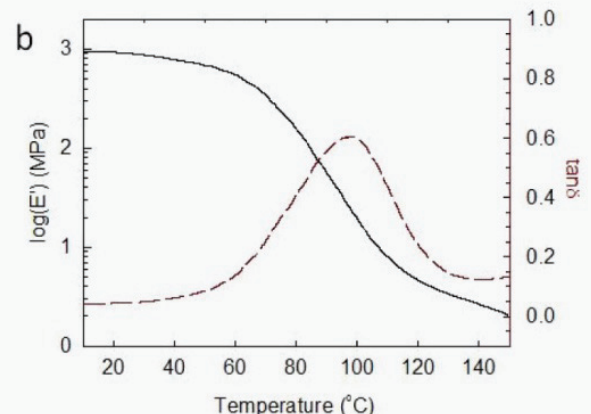

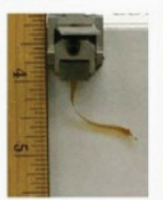

53

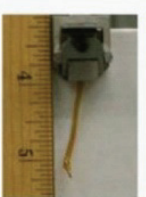

$\mathbf{5 2}$

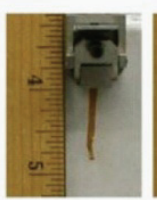

s1

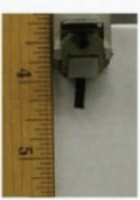

sorec

Fig. 15. The molecular structure, dynamic mechanical curve, and multi-SME of Nafion. (a). Molecular structure; (b). Dynamic mechanical analysis curve; and (c). Visual demonstration of a quadruple-SME [163], Copyright 2010. Reproduced with permission from Nature Publishing Group.

Mechanistically, we proposed a theory to explain this behavior. The critical point for this theory is that a single broad thermal transition can be regarded as the collective contribution of numerous infinitely sharp transitions continuously distributed in the broad temperature range. Each of these sharp transitions can be viewed as an elemental memory unit (EMU) corresponding to an infinitely sharp transition temperature. Based on this theory, at any $T_{\mathrm{d}}$, only the EMUs with their $T_{\text {trans }} \mathrm{s}$ below this particular $T_{\mathrm{d}}$ are activated for the shape memory function. For a single broad transition, the distribution of the EMUs is continuous. Thus, any $T_{\mathrm{d}}$ within this broad transition would correspond to a particular group of activated EMUs. Performing a triple-shape programming at any two deformation temperatures would always activate two different groups of EMUs for fixing. More importantly, these two EMU groups can be distinctively activated during reheating for two recovery events. Overall, this led to a tunable triple-SME. The same principle could be extended to a tunable multi-SME beyond triple.

Closely related to the tunable multi-SME is the so-called temperature memory effect (TME). The TME was first discovered in 2007 for a polyvinyl alcohol (PVA)-carbon fiber composite exhibiting a broad glass transition [164]. The composite was first deformed at a $T_{\mathrm{d}}$ within the glass transition. After cooling to fix the strain, $T_{\mathrm{d}}$ could be traced back as it was equivalent to $T_{\sigma_{\max }}$ observed in the corresponding recovery event run under an iso-strain 
condition. In essence, the material memorized $T_{\mathrm{d}}$. Thus, $T_{{ } \max } \approx T_{\mathrm{d}}$ establishes the basis for the TME. More precisely, this can be called stress based TME since the TME was reflected in the stress recovery event. The same effect was later confirmed by us and Lendlein's group for different polymers also with a broad thermal transition, confirming its generality $[165,166]$. While intriguing, the stress based TME may not be particularly relevant since most SMP applications relying on strain recovery instead of stress recovery. With this in mind, we designed a series of experiments in an attempt to establish a strain based TME for Nafion. The result of our investigation showed that, when the programming was conducted in a strain controlled condition, $T_{\mathrm{sw}}$ obtained from the corresponding strain recovery curve can indeed be quantitatively related to $T_{\mathrm{d}}$. Accordingly, the strain based TME was defined as $T_{\mathrm{sw}} \approx T_{\mathrm{d}}$. While the strain based TME was established for Nafion in our case, Lendlein's group observed the same behavior for a semi-crystalline network with a broad melting temperature $[167,168]$. Very recently, Zhou's group extended the TME to a $T_{\mathrm{m}}$-based biocompatible and biodegradable polymer [169]. In their work, four-arm PCL and linear PEG terminated with cinnamon groups were photo-crosslinked to yield a PCL-PEG network. By adjusting the ratio of PCL/PEG and the photo-irradiation time, a broad melting transition from 20 to $55^{\circ} \mathrm{C}$ could be obtained. Although such a transition range was not particularly broad, the authors were able to use small distinction of deformation/transition temperature (e.g., 37 and $45^{\circ} \mathrm{C}$ ) to achieve a TME.

While different from the tunable multi-SME, the TME can be readily explained by the same EMU theory. That is, a $T_{\mathrm{d}}$ corresponds to a particular group of activated EMUs for strain fixing. At the strain recovery event, this group of EMUs were all re-activated at the corresponding $T_{\mathrm{d}}$, leading to a maximum instantaneous strain recovery rate at this particular temperature $T_{\mathrm{sw}}$. Such a mechanistic discussion suggests that, although the tunable multi-SME and TME are not identical phenomenologically, they share the same molecular principle. This was supported by two independent modeling efforts by Huang's group and Qi's group $[170,171]$. For this reason, we chose to discuss them jointly in this section.

Because of Nafion's sophisticated ionomeric nature, it was not readily clear in the beginning if the mechanism proposed for the tunable multi-SME could be generalized for more common polymers. Collaborating with Luo's group, we synthesized a symmetric gradient copolymer of styrene (St) and methyl acrylate (MA) using a reversible addition-fragmentation chain transfer polymerization technique [172]. The gradient nature led to a broad glass transition between $20^{\circ} \mathrm{C}$ ( $T_{\mathrm{g}}$ of PMA homopolymer) and $100{ }^{\circ} \mathrm{C}$ ( $T_{\mathrm{g}}$ of PSt homopolymer). With the high $T_{\mathrm{g}}$ St segments at both ends of a chain and the low $T_{\mathrm{g}} \mathrm{MA}$ segments at the chain center, nano-domains with the highest $T_{\mathrm{g}}$ were formed. These domains acted as the physical netpoints. With this polymer, tunable multi-SMEs including quadruple- and quintuple-SMEs were observed. The work thus verified the generality of the tunable multi-SME and the underlying mechanism. Besides a single broad transition, the controlled polymerization technique could be used to precisely manipulate the glass transition(s) in an arbitrary fashion through controlling comonomer feedings. Thus, the methodology represents a highly attractive platform for synthesizing multi-SMPs.

Generality of the enabling mechanism allowed the tunable multi-SME to be realized in 
various other ways for a variety of polymers. In one instance, hydrophobic polyurethane (PU) chains were grafted onto hydrophilic PVA [173]. The hydrophobic PU domains acted as effective physical crosslinking points while the PVA-graft-PU retained the broad $\mathrm{T}_{\mathrm{g}}$ of PVA from $25{ }^{\circ} \mathrm{C}$ to $75{ }^{\circ} \mathrm{C}$, enabling a triple-SME with stability and recovery ratios all higher than $89 \%$. Moreover, the SME of the material could be triggered by water, since the $T_{\mathrm{g}}$ could be decreased when water molecules diffused into the polymer matrix. Other $T_{\mathrm{g}}$-type tunable multi-SMPs have also been developed. Linear PEG was immobilized in a crosslinked poly (methyl methacrylate) [PMMA] network, forming a PMMA/PEG semi-IPN [174]. The incorporation of PEG in the network not only introduced an additional $T_{\text {trans }}\left(T_{\mathrm{m}}\right.$ of PEG) but also broadened the $T_{\mathrm{g}}$ of PMMA, resulting in a quintuple-SME. In another example, norbornene cholic acid (NCA) and norbornene triethylene glycol monomethyl ether (NTEG) were copolymerized through a ring-opening metathesis polymerization [175]. Variation of the composition of NCA and NTEG within the copolymer allowed facile tune of material properties including the breadth of the $T_{\mathrm{g}}$ transition, hydrophilicity, solubility, and mechanical properties. The resulted norbornene-based copolymer displayed a broad $\mathrm{T}_{\mathrm{g}}$, enabling a quadruple-SME.

The preceding examples of tunable multi-SMPs rely on broad amorphous transitions. A broad melting transition was also found equally effective. An ethylene-vinyl acetate copolymer (EVA) typically possesses a relatively broad melting transition, with the $T_{\mathrm{m}}$ value determined by the comonomer ratio [165]. By blending two EVAs of different comonomer ratios, the breadth of the $T_{m}$ transition could be extended to $100{ }^{\circ} \mathrm{C}$. This was found sufficient to realize the tunable multi-SME. Weiss's group attempted to use the broad melting transition $\left(60 \sim 100{ }^{\circ} \mathrm{C}\right)$ of a commercial poly(ethylene-co-methacrylic acid) [PEMA] ionomer to achieve the multi-SME [40]. The PEMA were partially zinc-neutralized, forming ionic nanodomains as the physical crosslinks. Multiple temporary shapes could be well fixed with high $R_{\mathrm{f}} \mathrm{s}$, however, the $R_{\mathrm{r}}$ values were not particularly high probably due to the inefficiency of the ionic crosslinking points. When the ionomer was further crosslinked with electron beam, $R_{\mathrm{r}}$ was successfully improved to values approaching 100\%. In another example, ethyl cellulose was grafted with two PCLs of different molecular weights (7600 and 1400) [176]. The graft copolymers were mixed and subsequently crosslinked by MDI to form a permanent network. Instead of showing two separated $T_{\mathrm{m}}$, the material exhibited a broad $T_{\mathrm{m}}$ (from $34.4{ }^{\circ} \mathrm{C}$ to $92.7^{\circ} \mathrm{C}$ ), enabling a quadruple-SME. Intriguingly, a broad melting transition could also be achieved by doping with small molecules. Two paraffins with different $T_{\mathrm{m}} \mathrm{s}\left(32{ }^{\circ} \mathrm{C}\right.$ and $60{ }^{\circ} \mathrm{C}$, respectively) were incorporated into a physically crosslinked styrene-b-(ethylene-co-butylene)-b-styrene network. Within this network, the two paraffins exhibited a co-crystallization effect, yielding a broad $T_{\mathrm{m}}$ and a tunable multi-SME [177].

\subsection{Extrinsic multi-SMPs}

Practical constraints for many device applications limit access to direct heating. As a result, non-direct heating based triggering methods have attracted ever increasing attention in the general area of SMPs. As an added bonus, spatio-selective actuation is feasible for some non-direct heating based actuations (e.g., light). This enables temporal control of the recovery of a dual-SMP in multiple steps, resulting in an overall multi-shape behavior. Such behavior 
would fit into the broad definition of multi-SMPs, yet the molecular principle remains the same as dual-SMPs. We hereby suggest calling this type of systems extrinsic multi-SMPs, in distinction to the intrinsic multi-SMP for which the multi-shape behavior finds its roots in their more versatile molecular switch mechanism. Strictly speaking, it can be argued that these types of systems are not multi-SMPs. We will however illustrate below that extrinsic multi-SMPs are of equal (perhaps more) importance than intrinsic multi-SMPs, at least from the standpoint of potential device applications.

a

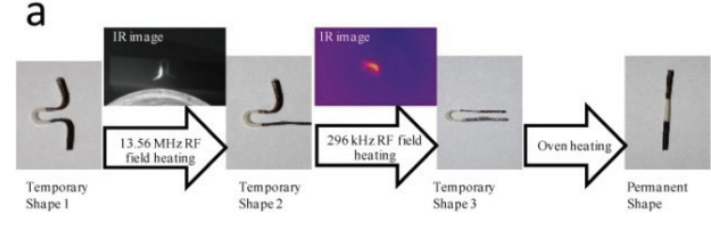

b

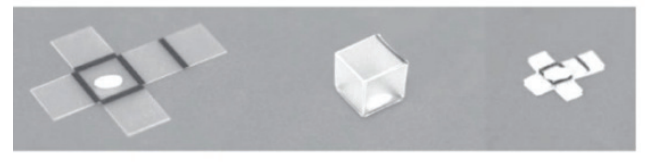

C

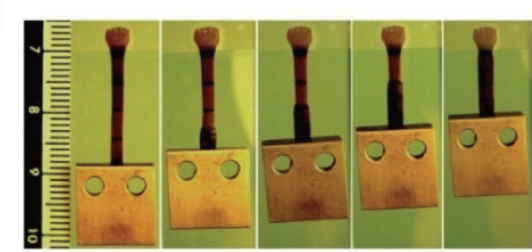

d

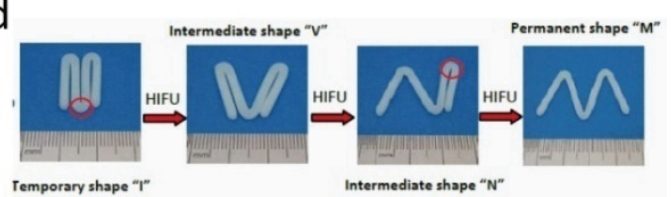

Fig. 16 Extrinsic multi-SMP systems. (a). RF selective actuation of a multi-composite [178], Copyright 2011. Reprinted with permission from John Wiley and Sons; (b). Local actuation by light followed by uniform heating of an SMP printed with black inks [179], Copyright 2012. Reprinted with permission from Royal Society of Chemistry; (c). Selective actuation by near IR of a gold SMP nanocomposite [90], Copyright 2012. Reprinted with permission from Royal Society of Chemistry; (d). Local actuation by focused high intensity ultrasound [95], Copyright 2012. Reprinted with permission from Royal Society of Chemistry.

Iron oxide particles are known to induce heating when exposed to an $\mathrm{AC}$ magnetic field. Incorporating them into an SMP is a known practice to fabricate a SMP composite for which the shape recovery can be actuated remotely via AC magnetic field. In a recent collaboration with Hilt's group, we discovered that carbon nanotubes can also induce heating, importantly at a much higher radiofrequency than iron oxide [178]. The principle that different nanofillers respond to an $\mathrm{AC}$ magnetic field (or radiofrequency (RF) wave) of different frequencies can be utilized to achieve multi-shape behavior. The proof of concept is illustrated in Fig. 16a with a multi-composite containing three segments of an iron oxide SMP composite, a carbon nanotube SMP composite, and a neat SMP. Here, the SMP matrix in the three segments does not need to be different. The multi-composite sample in a straight strip form (permanent shape) was deformed into a “ $\Omega$ ” shape in which all three segments were deformed. When the sample was exposed to a high RF, the carbon nanotube composite segment was recovered. At a low frequency, iron oxide composite segment was recovered. The neat polymer segment was recovered by direct heating. Following this particular actuation sequence, a total of four shapes were observed in a single shape memory cycle. Conceptually, one can alter the sequence of the actuations. As a result, the recovery of the temporary " $\Omega$ " shape to the permanent shape can follow five different routes involving a total of five shapes. Overall, the behavior of this extrinsic multi-SMP is drastically more versatile since the recovery of an intrinsic multi-SMP 
always follows a particular order predetermined in the programming step (i.e., from the low temperature shape to the high temperature shape, and to the permanent shape).

Shrinky-Dinks film is a well known toy that shrinks upon microwave heating. This behavior is a typical dual-SME and the Shrinky-Dinks film is essentially is a dual-SMP film in its temporary (biaxially stretched) state. Utilizing the Shrinky-Dinks film, Liu et al. [179] achieved the multi-shape capability by combining selective light triggering and non-selective direct triggering. Their simple yet elegant approach started by printing black ink on selective areas of the flat Shrinky-Dinks film cut into a particular shape (Fig. 16b). Upon exposure to infrared light, localized absorption of the light triggered heating and thus shrinking only on the printed areas, leading to the self-folding of the flat sheet into a three dimensional box. When the 3D box was further exposed to uniform direct heating, it unfolded back into a flat sheet that is proportionally smaller than the starting Shrinky-Dinks film.

The photothermal effect of nanoparticles has also been explored as the basis for light triggered shape recovery. Within the current context, Zhao and coworkers [90] fabricated an SMP nanocomposite by incorporating $\mathrm{Au}$ nanoparticles into a chemically crosslinked poly(caprolactone) network. Through the use of masks, laser light (wavelength: $532 \mathrm{~nm}$ ) was shined onto designated areas of the sample, triggering corresponding recovery. The spatio-selective nature of the light triggering allowed multi-stage recovery in a highly controllable manner (Fig. 16c). Along a similar line, they [95] demonstrated that high intensity focused ultrasound (HIFU) can also be utilized to induce heating in focused areas. As such, the recovery of the first temporary shape to the permanent shape can be manipulated step-wise to achieve multi-shape capability (Fig. 16d). As an added benefit, when drug molecules are incorporated in this SMP system, the multi-shape changes can be synchronized with controlled release profile of the drug.

Another unique extrinsic multi-SMP is the so-called functionally graded SMP, referring to a material that possesses a range of transition temperatures that are spatially distributed in a gradient fashion [180]. This material system was synthesized by a UV pre-curing at room temperature, followed by postcuring on a temperature gradient hot plate under the same UV source. Owing to the diffusion controlled reaction kinetics typical for a UV curing system, the $T_{\mathrm{g}}$ of the material is strongly tied to the curing temperature. As such, the temperature gradient on the hot plate resulted in the functionally graded SMP. When a deformed sample of the functionally graded SMP was exposed to continuous linear heating, spatially sequential recoveries were observed as the environmental temperature progressively exceeded the $T_{\mathrm{g}}$ 's. In a similar example, local $T_{\mathrm{g}} \mathrm{s}$ of a loosely crosslinked polymer film were spatially changed by immersing its different parts in a crosslinker solution with various concentrations [181]. The higher concentration of the crosslinker was applied, the higher local $T_{\mathrm{g}}$ could be obtained.

Instead of spatially gradient chemical structure, spatially distributed apparent $T_{\text {trans }}$ can be realized within a magnetic nanoparticles/SMP composite. As revealed in Sention 3.2, endogenous heating allows adjustment of $T_{\text {sw,app }}$ [96]. For samples with an identical chemical composition, smaller samples with higher specific surface areas provide higher $T_{\mathrm{sw} \text {,apps }}$ and vice versa. In this case, a sample with a heterogeneous geometry provided locally distinct $T_{\text {sw,apps, }}$, enabling the extrinsic multi-SME [182]. 
As stated in the preceding, the multi-shape behavior of an intrinsic multi-SMP can only be achieved via controllable heating methods (either staged heating or slow linear heating). As such, the fact that most of the extrinsic multi-SMP systems were realized by easily controllable remote triggering methods is advantageous. Despite the versatility of the various extrinsic multi-SMP concepts, however, one has to be mindful of their limitations. For instance, a common limitation of an extrinsic multi-SMP lies in its spatio-selective nature. That is, they are not suitable for devices that require multi-recovery on the identical spots in the sample. In addition, each extrinsic multi-SMP concept has its own practical limitation(s). Potential devices based on the selective radiofrequency actuation may require access to bulky induction heating equipment. Light triggering, on the other hand, is not particularly suitable for three dimensional bulk devices due to the requirement for direct exposure. For HIFU triggering, it may not have sufficient spatial resolution needed for miniature devices.

As is always true for the SMP field, there is not a single system suitable for all applications as each system has its prons and cons. The diversity of the various extrinsic and intrinsic multi-SMP concepts, on the other hand, supplies different options that can be picked upon for a particular application. To this end, one particularly interesting area of future development may lie in new systems that combine the intrinsic and extrinsic multi-SMP concept. This may overcome some of the limitations while providing enormous opportunities for designing easily controlled highly versatile SMPs.

\subsection{Reprocessible multi-SMPs}

Whereas thermoplastic multi-SMP naturally allow reprocessing to create new permanent shapes $[163,172]$, a number of other approaches based on reversible covalent and non-covalent bonds have also been explored to achieve the same goal.

A recent collaboration between $\mathrm{Ji}$ and Terentjev explored a highly intriguing idea of resetting the permanent shape of a triple-SMP via dynamically exchangeable covalent bonds [183]. In their work, a liquid crystal elastomer (LCE) with ample ester linkages in the network was synthesized. The LCE possessed a liquid-crystal isotropic transition temperature $\left(T_{\mathrm{i}} \approx\right.$ $\left.100{ }^{\circ} \mathrm{C}\right)$ and a glass transition $\left(T_{\mathrm{g}} \approx 55^{\circ} \mathrm{C}\right)$. With the addition of triazobicyclodecene (a transesterification catalyst) in the LCE, a transesterification reaction could be activated at a vitrification temperature $\left(T_{\mathrm{v}}\right.$, typically higher than $\left.160{ }^{\circ} \mathrm{C}\right)$. Below the $T_{\mathrm{v}}$, the LCEs were able to exhibit a triple-SME utilizing the $T_{\mathrm{g}}$ and $T_{\mathrm{i}}$ phases. Above $T_{\mathrm{v}}$, the ester linkages would break and reform in a dynamic fashion. Under this condition, when an external force was applied, the network topology was rearranged to release the stress. In essence, such a stress free deformed shape would represent a new permanent shape.

In a more conventional way, thermal reversible covalent bonds such as the Diels-Alder reaction were also applied to enable reprocessability for a triple-SMP. This was demonstrated by Yang and coworkers [184]. Crystalline poly(tetramethylene oxide) (PTMEG, $T_{\mathrm{m}}=25{ }^{\circ} \mathrm{C}$ ) and poly(p-dioxanone) (PPDO, $T_{\mathrm{m}}=105{ }^{\circ} \mathrm{C}$ ) with furan groups on both ends were first synthesized. The two polymers were subsequently crosslinked to form a single network via the Diels-Alder reaction between the furan groups and the maleimide groups from a tri-functional crosslinker [tris(2-maleimide ethyl)amine]. Whereas the two $T_{\mathrm{m}} \mathrm{s}$ from PTMEG and PPDO 
provided the basis for a triple-SME, heating to a higher temperature allowed decrosslinking for reprocessing via the reverse Diels-Alder reaction.

Photo-reversible covalent bonds in principle should also allow reprocessing of a triple-SMP. In an effort by Zhou and coworkers [169], PCL-diol and N,N-bis (2-hydroxyethyl) cinnamamide was copolymerized with MDI to yield a linear polyurethane with a $T_{m}$ around $40{ }^{\circ} \mathrm{C}$ and a $\mathrm{T}_{\mathrm{g}}$ around $70{ }^{\circ} \mathrm{C}$. The presence of the cinnamon groups allowed $\mathrm{UV}$ crosslinking $(\lambda=356 \mathrm{~nm})$ to introduce the netpoints, resulting in a triple-SMP. In theory, the photo-reversibility of the netpoints should permit resetting the permanent shape, although the author did not report such an attempt.

\section{Two-way SMPs}

The various SMEs described so far in this review belong to the one-way SME (1W-SME). Within a particular one-way shape-memory cycle, the shape recovery step can only go from the temporary shape(s) to the permanent shape. That is, unless the SMP is mechanically deformed again via a re-programming process, the recovered permanent shape cannot revert back to the temporary shape by altering the external stimulus only. Thus, 1W-SME is often time also referred to as an irreversible SME. Within the broad examples of SCPs, many polymers exhibit reversible shape changing behavior. Typically however, once the geometry is fabricated, an SCP can only switch between two fixed equilibrium shapes. We call this type of polymers as non-programmable reversible SCPs, in contrast to the 1W-SMP for which the temporary shape can be arbitrarily defined via programming. While most SMEs are one-way, it is of no surprise that scientists working in this field have been trying to develop polymers that can exhibit two-way SME (2W-SME) or reversible SME. In contrast to the non-programmable reversible SCPs and the programmable but irreversible $1 \mathrm{~W}-\mathrm{SMPs}$, a 2W-SMP takes best attributes of both and is defined as a polymer that can reversibly switch between two shapes that can be thermomechanically programmed and reprogrammed. Thus, a $2 \mathrm{~W}$-SMP may also be referred to as a programmable reversible SCP. The history and the latest development on 2W-SMPs are reviewed in this section.

\subsection{Quasi 2W-SME for liquid crystalline elastomers}

As early as 1975, de Gennes [185] predicted liquid crystalline elastomers (LCEs), that were not synthesized until 1981 by Finkelmann's group [186]. LCEs are elastic polymer networks containing main-chain or side-chain liquid crystal units that are capable of undergoing reversible mesomorphic-to-isotropic phase transitions. The liquid crystalline domains are typically randomly oriented with respect to each other, they are thus called liquid crystalline polydomains. The liquid crystalline domains can be aligned in a certain direction when an external field (e.g., magnetic field, stretching force) is applied during the formation of a LCE network [187-190]. This results in the so-called liquid crystalline monodomains in which the polymer chains are aligned and exhibit an extended chain conformation. Macroscopically, it corresponds to an elongated shape. When heated to a temperature above the liquid crystal clearing temperature $\left(T_{\mathrm{cl}}\right)$, the polymer chains would reduce its anisotropy, leading to macroscopic contraction in the sample length. Upon cooling below $T_{\mathrm{cl}}$, the sample would 
revert to the original anisotropic state and elongates. Between the elongated state and the contracted state, a large strain change of c.a. $90 \%$ can be observed [191]. Very importantly, this cooling induced elongation and heating induced contraction is fully reversible. This is an early example in which the reversible shape change originated from a thermal phase transition. However, there was no demonstration of manipulating the shape changing event via a programming step. Despite its non-programmable nature, this formed the basis for the subsequent development on 2W-SMPs.

Mondomains can be formed either chemically or physically. During the synthesis of a LCE, a two-step crosslinking method could be employed [187-191]. In the first step, an isotropic network is established via partial crosslinking. The network is then deformed to induce anisotropy, subsequently fixed by further crosslinking reactions in the second step. Alternatively, a one-step crosslinking method can also be used. Small molecular or polymeric liquid crystal precursors are macroscopically oriented by applying an external field (e.g., mechanical, magnetic, or electric field) [187-189]. Afterwards, the aligned precursors are polymerized/crosslinked to form a macroscopically anisotropic LCE. Notably, physical methods can also be employed to induce monodomain formation after the LCE polydomains are synthesized. Applying an external stress via hanging a weight is the easiest and most commonly used method to physically induce the monodomain formation [192,193]. A critical difference between the chemically induced and the physically induced anisotropy is that while the former cannot be altered after the sample fabrication, the latter can be tuned by changing the external stress (e.g., weight). Thus, the degree of anisotropy and the corresponding shape changing induced by the physical method can be tuned. In other words, the shape changing becomes programmable. We note here that, due to the need of an external force, the loose definition of "programmable" is used as will be clarified latter in this section. Nevertheless, the associated behavior can be called quasi 2W-SME. Not surprisingly, the combination of chemical and physical domain orientation methods (i.e., applying an external stress onto a chemically oriented LCE) led to high anisotropy and a reversible strain change as high as $400 \%$ $[194,195]$.

\subsection{Quasi 2W-SME for semi-crystalline networks}

Despite the intriguing shape changing behavior observed for LCEs, they are difficult to synthesize and the transition temperatures are not easy to tune. In 2008, Mather's group [196] reported an important discovery, demonstrating that a semi-crystalline polymer network exhibited a similar behavior. In the presence of a constant tensile load, a crosslinked poly(cyclooctene) $[\mathrm{PCO}]$ semi-crystalline network underwent elongation when they were cooled across the $T_{\mathrm{m}}$ (i.e., crystallization induced elongation or CIE) as schematically shown in Fig. 17a. When heated above the $T_{\mathrm{m}}$ under the same load, the elongation was reversed (i.e., melting induced contraction or MIC). Similar to LCEs, the extent of reversible strain change was also tunable via the adjustment of the constant external stress. Subsequent investigations by other groups suggested that this behavior is generic for semi-crystalline networks [197-202]. In particular, Lendlein's group in 2010 reported a two-way reversible triple-shape system in which a semi-crystalline network with two distinct melting transitions could reversibly switch 
a

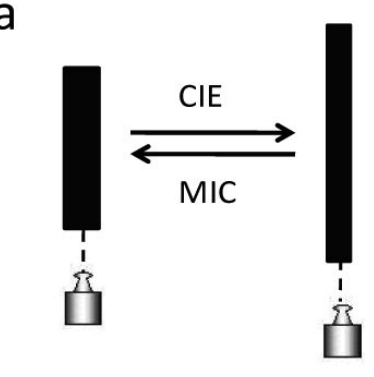

b

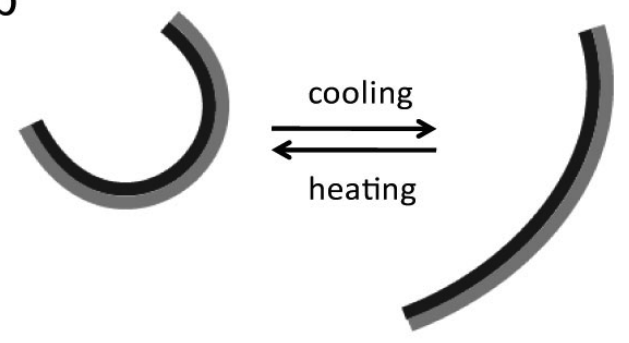

C
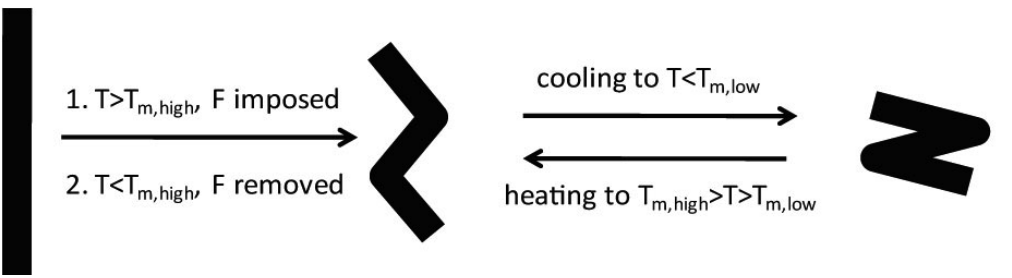

Fig. 17. Schematic illustration of various 2W-SMEs: (a). crystallization induced elongation (CIE) and melting induced contraction (MIC) under a constant stress; (b). Reversible bending action of a bilayer composite under zero external stress: the black layer represents a pre-stretched SMP and the gray layer represents an elastomer; (c). Programmable 2W-SME.

The achievement of the CIE-MIC transformation for the preceding semi-crystalline networks all required the presence of an external force, albeit constant. Looking back to the mechanisms behind LCEs, however, perhaps the anisotropic alignment of the polymer chains was the true underlying mechanism for semi-crystalline polymer networks. Nevertheless, it was the external stress that allowed tuning the anisotropy and the strain change. However, the requirement for an external stress is a serious limitation for the potential device applications based on this type of behavior. The search for alternative mechanisms and materials to enable a $2 \mathrm{~W}-\mathrm{SME}$ without the need for an external stress (i.e., true $2 \mathrm{~W}-\mathrm{SME}$ ) has been a constant pursuit in the SMP community. Along the way, several contributions are particularly noteworthy.

Hu's group fabricated an SMP bilayer by bonding a pre-stretched $T_{\mathrm{m}}$-type shape memory polyurethane with a non-stretched polyurethane elastomer [204,205]. Upon heating above the $T_{\mathrm{m}}$ of the SMP layer, the bilayer bended towards the direction of the SMP. The bending angle was significantly reduced when the sample was cooled below the $T_{\mathrm{m}}$. The procedure is schematically shown in Fig. 17b. Importantly, the change in the bending angle was fully reversible. Notable differences from the examples discussed in the last paragraph are: the lack of an external stress; reversible bending versus reversible elongation/contraction. Analyzing the details a bit deeper, one might realized that the shape mismatch between the elastomer layer and the SMP might have served the role of the external stress. Specifically, the SMP tended to shrink upon heating while the elastomer provided an internal force to resist its shrinking. In essence, an internal tensile stress was generated on the SMP. Because of the macroscopic bilayer construction, the direction of this internal stress was such that it caused bending instead of elongation/contraction. Here, it was this internal stress that induced the 
network anisotropy for the semi-crystalline SMP, thus enabling a CIE effect. Due to the elongation of the SMP layer upon cooling, the bilayer reduced its bending angle. More recently, Qi and Mather fabricated their own bilayer system with crosslinked semi-crystalline poly(cyclooctene) as the active SMP layer and silicon rubber as the inactive elastomer layer [206,207]. Despite the difference in material constituents, the bilayer showed a similar reversible bending behavior. For both bilayer systems, the shape changing was reversible, but the shapes involved cannot be programmed since the internal stress was determined in the fabrication process and cannot be altered afterwards. The big questions were: can a mechanism be perceived to enable the alternation of the internal stress after the fabrication; if so, will it lead to a programmable reversible shape changing behavior without the need for an external stress, that can be defined as a $2 \mathrm{~W}-\mathrm{SME}$ in a strict sense?

\subsection{W-SME for semi-crystalline networks}

In 2013, Lendlein's group devised an elegant approach that answered both questions posed in the preceding section in a positive way. In their work [33], a polyester urethane (PEU) network with poly( $\omega$-pentadecalactone) [PPDL] and PCL segments was synthesized. The two polyesters provided a high $T_{\mathrm{m}}\left(T_{\mathrm{m}, \text { high }}\right)$ around $64{ }^{\circ} \mathrm{C}$ and a low $T_{\mathrm{m}}\left(T_{\mathrm{m}, \text { low }}\right)$ around $34{ }^{\circ} \mathrm{C}$, respectively. Whereas the PEU network is an intrinsic triple-SMP due to the two $T_{\mathrm{m}} \mathrm{s}$, this time they illustrated a $2 \mathrm{~W}$-SME following the procedure outlined in Fig. $2 \mathrm{~b}$. The original PEU sample (permanent shape $\mathbf{C}$ ) was first deformed at a $T_{\text {reset }}>T_{\mathrm{m} \text {,high }}$ by the application of an external force. The deformation was then fixed at a $T_{\text {low }}<T_{\mathrm{m} \text {,low }}$. Upon stress removal, a shape B was obtained. This completed the programming step, that was actually identical to the programming step encountered in a typical one way shape memory cycle. However, it was at this stage that the rest of shape memory cycle became different. The PEU sample was reheated to a $\mathrm{T}_{\text {high }}\left(T_{\mathrm{m} \text {,low }}<T_{\text {high }}<T_{\mathrm{m} \text {,high }}\right)$, leading to another shape $\mathbf{A}$. What was truly intriguing was the discovery that the sample exhibited a reversible shape transformation between shape $\mathbf{A}$ and shape $\mathbf{B}$ when the temperature was cycled between $T_{\text {high }}$ and $T_{\text {low }}$. This established the reversible shape changing behavior without any external stress. What was equally important was the programmable aspect of the observed behavior. Upon heating to $T_{\text {reset, }}$, the sample would recover to the original shape $\mathbf{C}$. At this stage, a new programming step could start, through which the reversible switch between two new shapes $\mathbf{B}^{\prime}$ and $\mathbf{A}^{\prime}$ could be realized. As shown in Fig. 2b, a sample can be programmed to undergo multiple reversible shape switching between two shapes. It could be reprogrammed to reversibly switch between two completely different shapes, without any external stress present during the reversible shape switching. This was the first realization of a $2 \mathrm{~W}-\mathrm{SME}$ in its strict definition.

Mechanistically, the authors suggested that the PPDL domains associated with the $T_{\mathrm{m} \text {,high }}$ were used to determine the shape shifting geometry while the low temperature melting PCL domains were responsible for the reversible shape shifting. The deformed PPDL domains built a skeleton of crystallized geometry to orient the PCL domains. Conformational orientation of the PCL segments resulted in reversible geometric change of the PCL domains during crystallization and melting. Consistent with the preceding description, we hereby proposed a further mechanistic picture with the concept of network anisotropy discussed in this section. 

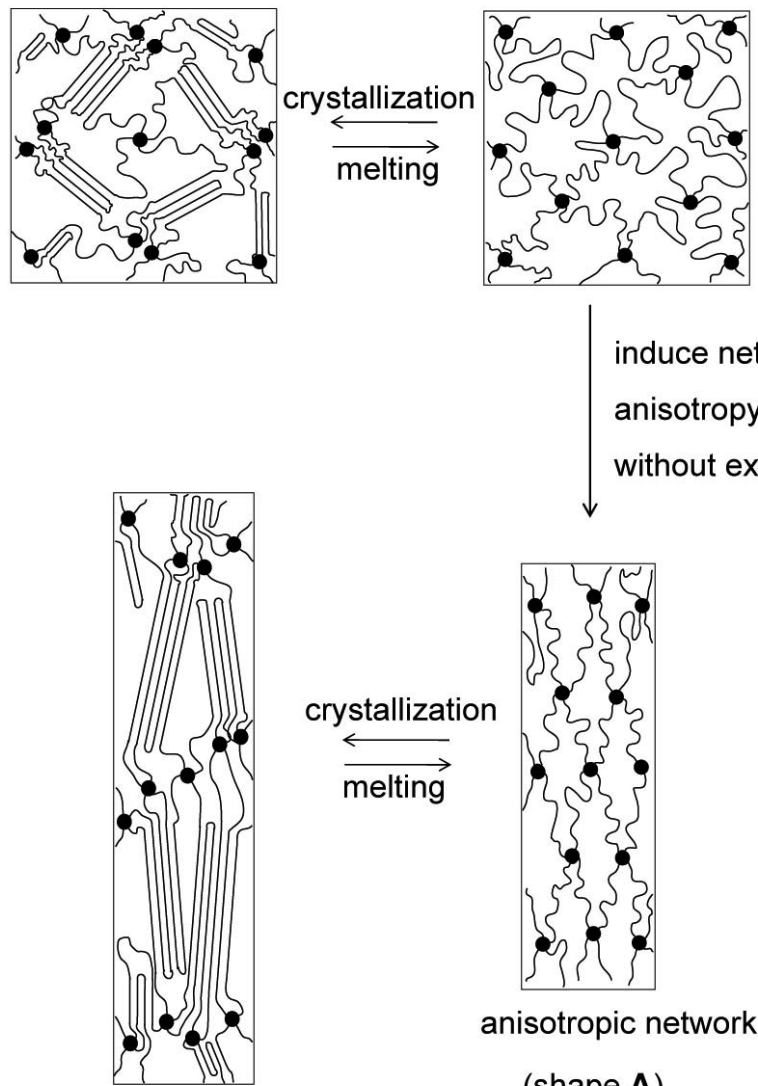

induce network anisotropy with or without external force

aligned crystals

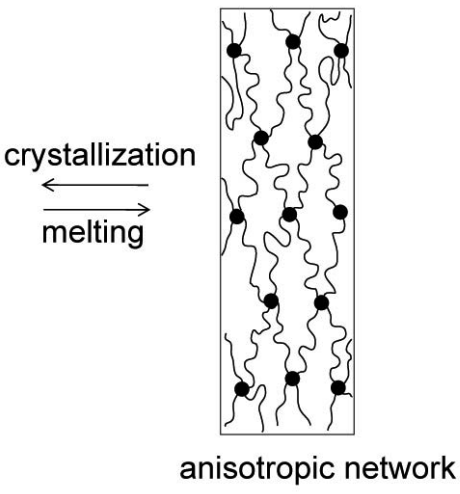

(shape A)

(shape B)

Fig. 18. The molecular mechanism for $2 \mathrm{~W}-\mathrm{SME}$ of a crosslinked crystalline polymer with an isotropic or anisotropic network structure.

This is outlined in Fig. 18. Beginning from the original shape $\mathbf{C}$ at $T_{\text {reset, }}$ the network chains adopt random chain conformation. Upon deformation during the programming step, the chain conformations associated with both crystalline phases were changed in shape $\mathbf{A}$. Reheating to $T_{\text {high }}$ removed only partially the orientation in the PCL chains. The deformation fixed by the PPDL domains was untouched, setting the network anisotropy for the PCL domains without external force, i.e. the PCL chains are in oriented conformation due to the constraint by the deformed PPDL domains. In this case, macroscopic CIE-to-MIC transformation of the PCL domains could be induced without external force. Therefore, the PEU network exhibits the $2 \mathrm{~W}$-SME. Overall, it is this internally created network anisotropy that was responsible for the reversible shape shifting. Since this network anisotropy was determined by the deformation during the programming step, the reversible shape switching can be reprogrammed. In a slightly different way, one may also view the high temperature PPDL phase as the phase that determines the internal stress that can be tuned via programming. Thus, the mechanism around network anisotropy or an internal stress unifies the different types of reversible shape switching behavior for the various materials discussed throughout this section.

In a different design, Lendlein's group extended the preceding material concept to a network exhibiting a broad $T_{\mathrm{m}}$ transition, instead of two distinct $T_{\mathrm{m}}$ transitions [208]. A network was established by blending EVA copolymers with different $T_{\mathrm{m}}$ followed by thermal 
crosslinking. This led to a single broad melting transition from $30{ }^{\circ} \mathrm{C}$ to $90{ }^{\circ} \mathrm{C}$. Whereas such a network was showed to exhibit a TME as mentioned before, it was found that the principle of the 2W-SME could also be applied here. According to the EMU theory in Section 6.1.3, a broad $T_{\mathrm{m}}$ could be regarded as the continuous distribution of a succession of countless and infinitely sharp $T_{\mathrm{m}} \mathrm{s}$. For the PEU network, $T_{\text {high }}$ should be between $T_{\mathrm{m} \text {,high }}$ and $T_{\mathrm{m} \text {,low }} T_{\text {high }}$ for this EVA system, however, could be randomly chosen within its broad melting transition. Once the $T_{\text {high }}$ is chosen, the group of EMUs with melting temperatures below this temperature would play a role similar to the low melting temperature phase in the PEU network. Similarly, the rest of the EMUs with melting temperatures above $T_{\text {high }}$ served the role of the high melting temperature phase. Thus, the preceding principle for the $2 \mathrm{~W}-\mathrm{SME}$ observed for the PEU network could be readily applied here for EVA network, except that $T_{\text {high }}$ could be any temperature within the broad melting transition. Experimental results confirmed that the 2W-SME could indeed be realized in this fashion. Depending on the chosen $T_{\text {high }}$, however, the fractions of the low melting temperature EMUs versus high melting temperature EMUs would change accordingly. This would quantitatively impact the 2W-SME. Along the same line, Sheiko's and coworkers [209] synthesized a poly(octylene adipate) network that also exhibited a broad melting transition. Via an identical mechanism but a different deformation mode, a surface 2W-SME was successfully demonstrated [210].

Pretsch and coworker [211], on the other hand, demonstrated a $2 \mathrm{~W}-\mathrm{SME}$ for a polyurethane elastomer. Upon deformation to a large strain, strain-induced crystallization occurred. Upon unloading, the authors discovered that the sample could undergo thermo-reversible elongation and contraction. Progressive growth of oriented crystallites during cooling was believed to be the mechanism behind such a behavior, as verified an in situ wide-angle X-ray scattering study. Besides linear elongation and contraction, geometrically more complex reversible shape switching (e.g., twisting) could also be realized by manipulation of the crystalline orders in the first deformation step. This initial deformation step was thus called a training process, the term commonly used for shape memory alloys. Being a different term, "training" was essentially "programming" used for $2 \mathrm{~W}-\mathrm{SMPs}$ in this section.

Despite differences in programming and the associated materials for the various $2 \mathrm{~W}-\mathrm{SMPs}$, the fundamental underlying mechanism is the same. The $2 \mathrm{~W}$-SME requires an internal way to create network anisotropy or an internal stress. This network anisotropy (or internal stress) should be erasable to allow reprogramming.

\section{Novel SMP fabrication methods}

Processing polymers into certain shapes is a necessary step towards their eventual uses. Fabrication of SMP devices in the relevant and potentially complex shapes is particularly important given the fact that their functions are much more tightly and uniquely bound to the shapes. In many device applications of SMPs, the involved materials cannot provide the intended functions without been processed into proper macroscopic three dimensional (3D) shapes or microstructured surfaces. In addition to traditional polymer processing methods (e.g., injection molding and liquid molding) that can be used to produce relatively simple shapes, 
several methods that lead to new SMP design, capability, and highly complex shapes have emerged. These methods are introduced in this section.

\subsection{Electrospinning}

Electrospinning is a versatile method for preparing complex two dimensional (2D) or 3D construction of fibrous assemblies [212]. Thermoplastic SMP can be processed via electrospinning and the method offers two benefits: fast recovery kinetics due to the porous structures that facilitate diffusion; the porous structures can be tuned via SME $[41,213]$. Mather's group extended the method to make a composite with shape memory functions by combining two non-SMP components [214]. A nonwoven fiber mat was formed by electrospinning of a thermoplastic crystalline polymer. The mat was then encapsulated with silicone rubber. The resulting composite exhibited shape memory functions with the polymer fibers as the switching phase, while the encapsulating rubber phase ensured the permanent shape. Such an approach is highly versatile in that it allows individual tuning of the properties of the two components, thus the overall shape memory behavior. For instance, when the fibers were aligned during the electrospinning process, the composite exhibited strong anisotropy in Young's modulus, strain-to-failure, yield stress, and shape fixing ratio $\left(R_{\mathrm{f}}\right)$ [215]. In addition to the predefined fiber alignment, isotropic SMP fibers can be temporarily oriented through shape memory programming. This enabled an isotropic/anisotropic transition that may be useful as dynamic tissue engineering scaffolds. The versatility of the method is also reflected in the making a triple-SMP composite by replacing the rubber encapsulating phase with a dual-SMP. This has been described in details in Section 5 .

\subsection{Four dimension printing}

3D printing has attracted significant interest lately due to its unlimited flexibility in terms of the geometric complexity of the fabricated parts [216,217]. This unique capability is quite complementary to shape manipulation via the shape memory programming. Thus, combining shape memory properties with $3 \mathrm{D}$ printing offers great potential in two aspects: producing SMP devices with practically relevant complex geometries that cannot be easily made by traditional processing methods; more shape variants can be realized for a 3D printed SMP part via shape memory programming. The time dependent SME offers an additional dimension (i.e., time), leading to the so-called four dimensional 4D printing. In principle, 4D printing can be realized in two ways: a thermoplastic SMP can be 3D printed directly using fusion deposition modeling; UV radiation curing of liquid SMP precursors. Qi, Dunn, and coworkers, however, reported an entirely different method [218, 219]. Using a 3D multimaterial polymer printer, an active composite was constructed with SMP fibers embedded in an elastomeric matrix. The orientation of the fibers was spatially controlled. Since the composite was printed in $2 \mathrm{D}$, its fabrication was fast relative to direct printing of multiple layers in 3D. Simple programming can lead to complex 3D configurations with spatially nonuniform curvature due to the controllable internal stress (Fig. 19). The curvature can be manipulated by adjustment of the stretching strain. Upon heating, the 2D sheet can be recovered. Overall, the most intriguing aspect of this technique is that it reduces significantly the fabrication time by printing in $2 \mathrm{D}$, 


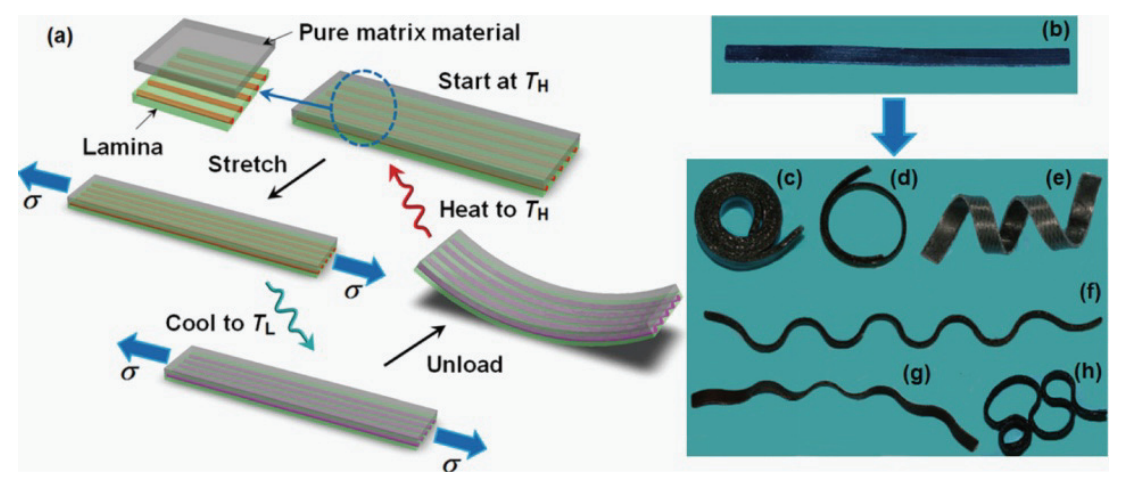

Fig. 19. Complex low-temperature shapes of 4D-printed laminates. (a) A two-layer laminate with one layer consisting of oriented SMP fibers embedded in an elastomer and the other layer being a pure elastomer matrix. The sample was heated, stretched, cooled, and released. Upon release of the stress it assumes a complex shape, depending on the laminate architecture. When reheating it assumes its original shape, a flat rectangular strip. (b) Shows an actual strip in its original shape and (c)-(h) show results of this process with differing fiber architectures [218], Copyright 2013. Reprinted with permission from AIP Publishing LLC.

\subsection{Photo-induced shape transformation}

Permanent shapes of chemically crosslinked polymers are defined by the topology of molecular network structure. By manipulating network topology, bulk shapes or surface morphologies of the polymers can be accordingly controlled. Bowman's group developed two methods that can locally vary network topology induced by photo irradiation, including photo-induced plasticity and two-stage curing. These two methods have been used to create complex shapes utilizing light induced and spatially defined internal stress.

Covalent networks have been synthesized incorporating allyl sulfide groups, providing the addition-fragmentation chain transfer capability (Fig. 20a) [220,221]. Due to the presence of a photo-initiator, light irradiation created radicals, that induced the chain transfer within the network. This introduced a dynamic character, potentially allowing alteration of the topology of the networks without changing the network composition and connectivity. When a sample was strained under a certain force and subsequently irradiated with spatially defined light through a photomask, the exposed regions underwent network rearrangement, that led to localized stress relief (or photo-induced plasticity) (Fig. 20b). As a result, the polymer chains in the exposed regions were in an unstrained state while those in the unexposed regions were still strained. After cessation of the external force, buckling features emerged due to the recovery in the unexposed regions (Fig. 20c). These features were determined by the mask design. In a similar demonstration, photo-reversible disulfide bonds were incorporated in a hydrogel network, resulting in a similar photo-induced rearrangement of network topology [222]. We note that the preceding two examples did not involve SMP as the subject of study. However, the same principle can be readily applied to SMPs. Indeed, Rowan group synthesized a polydisulfide network that exhibited both thermally-induced SME and 
photo-induced plasticity [223], the latter allowing manipulation of the permanent shapes. In their work, bisthiol oligomer synthesized via thiol-ene chemistry between 1,6-hexanedithiol and 1,5-hexadiene was crosslinked by tetrathiol in the form of disulfide bonds (Fig. 20d). The network exhibited a $T_{\mathrm{m}}$ around $61^{\circ} \mathrm{C}$ that enabled a thermally-induced SME. More importantly, the permanent shape could be repeatedly changed under UV light exposure. This allowed complex permanent shapes to be obtained via deformation under photo-irradiation, instead of traditional molding or remolding (Fig. 20e).

a
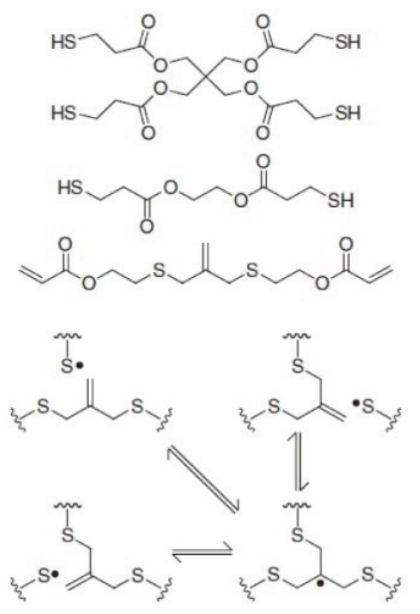

d

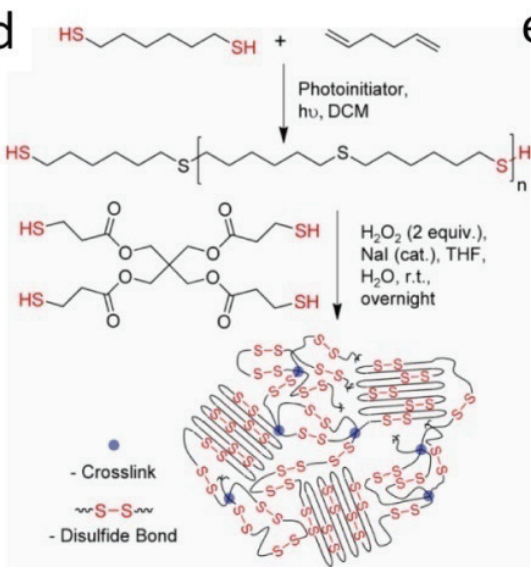

b

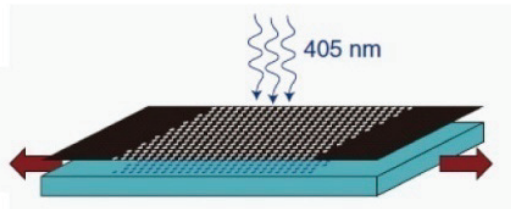

C

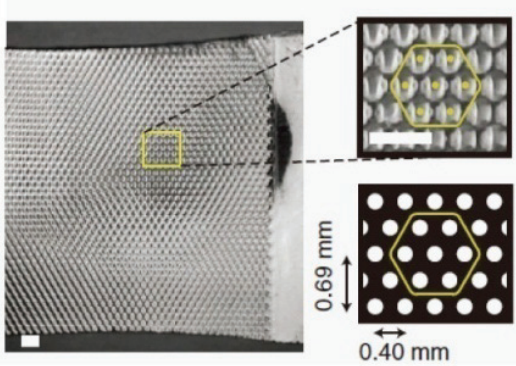

e

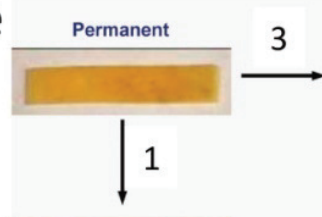

Reprogrammed
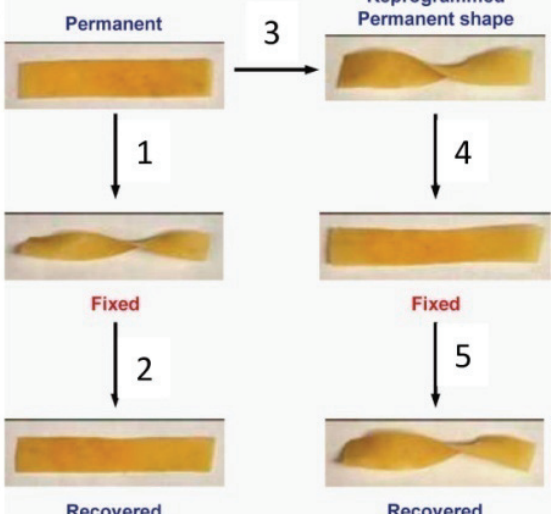

Recovered

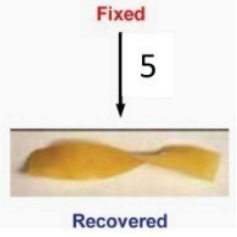

Fig. 20. Photo-induced complex shape transformations. (a). Monomers and reaction mechanism for the addition-fragmentation chain transfer within the polymer backbone; (b). Mechanism of the mechanophotopatterning: the material is uniaxially stretched and UV irradiated though a mask; (c). The hexagonal pattern obtained after process (b) (scale bar $=1 \mathrm{~mm}$ ) [221], Copyright 2011. Reprinted with permission from John Wiley and Sons; (e). Synthesis of the semicrystalline polydisulfide network; (f) Visual demonstrations showing the reprogrammable shape memory properties of the polydisulfide network: ( 1 and 2$)$. A flat specimen is fixed into a helical shape and subsequently recovered to the permanent flat shape; (3). The permanent shape of the film can be photo-reprogrammed by holding it in the helical shape while exposing it to UV light; (4 and 5). Programming and recovery of the new permanent shape [223], Copyright 2013. Reprinted with permission from the American Chemical Society. 
Besides photo-induced plasticity, a two-stage-curing technique can also be applied to manipulate SMP permanent shapes [224]. It refers to dual curing reactions that can selectively occur to form two types of netpoints within a polymer network. From a non-stoichiometric thiol-acrylate system possessing excess acrylate groups, a first polymer network can be formed via a base catalyzed stoichiometric thiol-acrylate Michael addition (stage I). The excess acrylate groups can be further polymerized upon UV irradiation (stage II). In stage II, if the UV irradiation was locally applied on a strained first network, the strained network topology in the exposed regions would be permanently fixed by the newly formed netpoints. In contrast, the unexposed regions would recover to its initial state upon relief of the external force. As a result, light-defined buckling 3D features could be formed. In another work [225], a stoichiometric thiol-isocyanate system with excess reactive acrylate groups was used to construct a first network at stage I. By controlling the light irradiation time and intensity at stage II, intricate property gradients can be incorporated into the material. Due to the light attenuation along the penetration depth, the crosslinking density at stage II was significantly different between the surface and the interior of the material. Therefore, gradient modulus and $T_{\mathrm{g}}$ along the irradiation direction were endowed within the network. Due to the internal stress mismatch between the exposed and unexposed regions upon straining and subsequent heating induced stress relief of the bulk material, surface buckling or bulk geometric transformations could be achieved. Similarly, electron beam irradiation was also applied to manipulate the crosslinking density in order to finely tune material properties such as rubbery modulus, strain capacity, ultimate tensile strength, recovery stress, and glass transition temperature.

\subsection{Origami-inspired manufacturing}

Complex 3D structures may also be created via folding a 2D sheet, the essence of the origami art. Locally-induced shape recovery of a SMP sheet can lead to a self-folding effect. This technique, so-called origami-inspired manufacturing, is attractive for a number of applications such as packaging and mechanical actuation.

Dickey's group demonstrated the self-folding of an SMP sheet triggered by infrared heating [179]. The biaxially stretched sheet in its neat SMP form was optically transparent and displayed in-plane shrinkage upon uniform heating. When black ink was locally patterned on one side of the sheet, the patterned regions could absorb optical energy to heat the underlying SMP. The light induced heating is mostly limited to the patterned regions on one side of the sheet. As a result, the patterned side shrunk while the opposite side did not, leading to an overall self-folding effect. This intriguing approach is simple and efficient, however, the folding in different regions can only be simultaneously triggered. In contrast, Felton et al. [226] developed a method that could achieve sequential self-folding. Composite sheets were constructed by laminating three layers: a pre-stretched SMP, a piece of scored paper, and resistive copper traces (as shown in Fig. 21). Heating induced shrinkage of the SMP layer caused the laminate to bend. The bending behavior would only occur at the scored location via joule heating of the copper traces, leading to localized folding. Moreover, the fold could be triggered simultaneously or sequentially upon appropriate on-off control of the copper traces. Through the origami-inspired approach, 3D complex structures can be created by an efficient 
2D printing technique. Parameters within the 2D fabrication can be modeled and controlled, because the folding behavior (the maximum fold angle) is strongly affected by the composite thickness, the score depth, and the trace width.
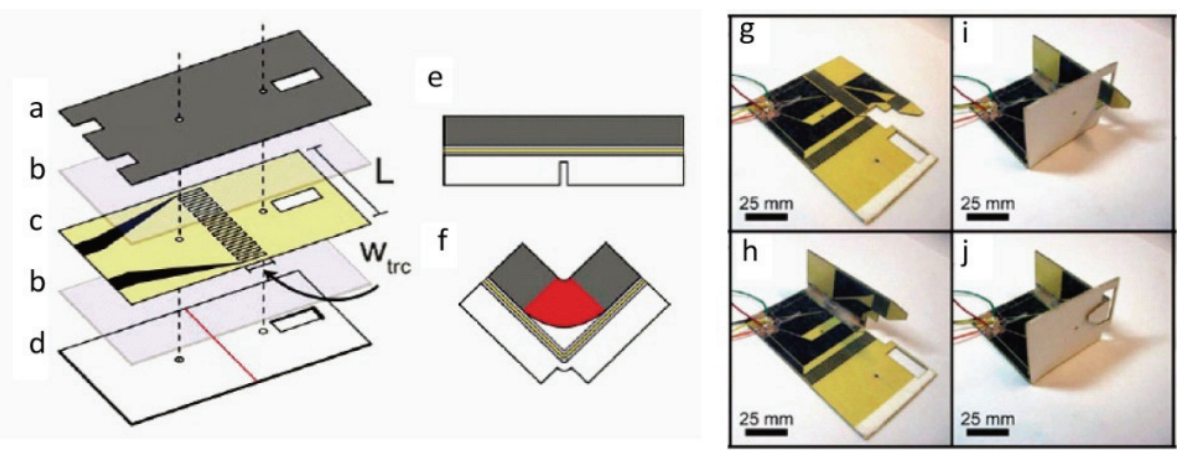

Fig. 21. The construction and operating mechanism of a self-folding composite. (a) an SMP layer, (b) silicone tapes, (c) a polyimide sheet with copper trace, and (d) a paper substrate scored along the hinge in the middle; (e and f) The composite bends at the hinge due to the contraction of the SMP layer upon activation; $(g-j)$ A locking slot and tab assembly. This fold is only possible with sequential folding [226], Copyright 2013. Reprinted with permission from Royal Society of Chemistry.

\section{Modeling of thermomechanical behavior of SMPs}

As being realized by many researchers, the SME is a response of materials under a special set of thermomechanical loading conditions, rather than a material property. Therefore, SME is determined not only by material properties, but also by complicated thermomechanical loading conditions. In many cases, the latter complicates the evaluation of shape memory performance as well as material design. For example, material properties, such as glass transition temperature and the width of glass transition, affect the shape stability and shape recovery; in addition, these features may also be affected by the temperatures at which the material is deformed and the shape is fixed [227-232]. Therefore, it is well recognized that SME modeling should play an important role in guiding the design of SMP applications as well as the design of materials for specific applications.

In SME modeling, one approach is to develop constitutive models (or thermomechanical constitutive models) for the polymer. The expectation is that one can then use such models, together with simulation tools, such as finite element methods, to simulate the shape memory behavior. Another approach in SME modeling is to directly predict shape memory performance. Here, although the model development is based on certain assumption of constitutive models for polymers, the end result is an analytical solution that can direct predict the shape memory performance, such as shape stability or shape recovery.To date, the majority of modeling efforts have been on the development of constitutive models for SMPs; very few models have beem developed in the second group.

In continuum mechanics, constitutive models are defined as equations that link state functions, such as internal energy, entropy, heat flux, and Cauchy stress, to state variables, such as deformation gradient, temperature, etc., sometimes with the help of internal variables. 
From such a definition, it is clear shape memory effects are an exhibition of constitutive behavior under complicated thermomechancial conditions. Therefore, constitutive models of shape memory polymers can sometimes also be found in those for polymers going through thermomechanical transitions. For example, in a recent work by Srivastava et al. [233], shape memory behavior are naturally captured under the framework of a constitutive model of polymers for finite deformation behavior spanning across glass transition temperature [234].

A large number of constitutive models have been developed for SMPs and several excellent reviews are available for these models [9, 235-237]. It is therefore not necessary to repeat the review of these models again. Below, we will focus on describing the prevailing concepts in constitutive modeling as well as some efforts in using simple models to estimate shape memory performance.

\subsection{Thermoviscoelastic models}

Amorphous polymer based SMPs consist of a large portion of SMPs currently studied, and are therefore heavily investigated. The molecular mechanism of this group of SMPs is the large chain change in chain mobility during the glass transition [5]. From polymer physics or mechanics point of view, this chain mobility change is reflected in the change of relaxation time or viscosity [238-241], encompassed in viscoelastic behavior. A good discussion on how shape memory effect is an outcome of temperature-dependent viscosity can be found in a few recent work [171,236,237]. It is therefore natural to use viscoelastic models where the viscosity is a function of temperature, or the so-called thermoviscoelasticity, to capture the shape memory effect. In the early models developed by Tobushi and co-workers [242-244], viscosity and relaxation time were not explicitly written as functions of temperature. Later, the concept of thermoviscoelasticity was used by Nguyen et al. [245] and Castro et al. [227] where the relaxation time was considered to follow the principle of time-temperature superposition $[239,246]$ and the temporary shape is a direct result of frozen viscous strains in nonequilibrium elements. It should be noted that the phenomenological model frame developed by Liu and coworkers $[247,248]$ where the glass transition is considered as a phase transition served as the basisfor many modeling works in recent years [249-256]. In many of these models, a storage strain (initially used by Liu et al. [247]) was also used as a mechanism to capture the shape memory effect. In addition, the slip element for internal friction for shape memory effect by Tobushi and co-workers [242-244] was recently used for 3D models [257-264].

One advantage of thermoviscoelastic models is that it closely follows the underlying polymer physics for shape memory effects in amorphous SMPs and thus can be easily used to consider other effects, such as aging and solution driven SME, into constitutive model. For example, when both time-temperature dependent structural relaxation (or physical aging) and stress relaxation are were considered, Choi et al. [265] showed that the initial shape recover speed can be improved. Li and co-workers [266] also used the thermoviscoealstic model to investigate a SMP based syntactic form. It is known that water (or solution) can be used as a means for shape memory effect $[52,267]$, because small molecules, such as water, can serve as plasticizers to decrease the glass transition temperature, or effectively increase the relaxation time, effects included in models by Xiao and Nguyen [268] and Lu and coworkers [269, 270]. 
For example, in Xiao and Nguyen, the structural relaxation time can be written as

$$
\tau_{R_{k}}\left(T, T_{f}, \theta\right)=a\left(T, T_{f}, \theta\right) \tau_{R_{k}}\left(T, T_{f}, 0\right),
$$

where $\theta$ is the solution concentration, $T$ is the temperature, $T_{f}$ is the fictive temperature of the nonequilibrium structure, $a\left(T, T_{f}, \theta\right)$ is a temperature-dependent solution-shifting factor, $\tau_{R_{k}}\left(T, T_{f}, 0\right)$ is the dry-state relaxation time,

$$
\begin{gathered}
a\left(T, T_{f}, \theta\right)=a^{-1}\left(T, T_{f}, 0\right) \exp \left\{\frac{B \zeta^{-1}}{T\left(C \zeta^{-1}\left[T_{2}^{-1}-T_{f}^{-1}\right]\right)-[\theta \ln (\theta)+(1-\theta) \ln (1-\theta)]}\right\}, \\
a\left(T, T_{f}, 0\right)=\exp \left\{\frac{B}{T\left(C\left[T_{2}^{-1}-T_{f}^{-1}\right]\right)}\right\}, \\
\tau_{R_{k}}\left(T, T_{f}, 0\right)=\tau_{R_{k}}^{g} \exp \left[-\frac{C_{1}^{g}}{\log (e)}\left(\frac{c_{2}^{g}\left(T-T_{f}\right)+\left(T_{r}-T_{g}^{r e f}\right)}{T\left(C_{2}^{g}+T_{r}-T_{g}^{r e f}\right)}\right)\right],
\end{gathered}
$$

where $\tau_{R_{k}}^{g}$ is the relaxation time at an equilibrium reference temperature at dry state, $T_{2}$ is the Kauzmann temperature for an ideal glass. Note the exponential term in Eq. $5 \mathrm{c}$ is the shifting factor for time-temperature superposition, where $C_{1}^{g}$ and $C_{2}^{g}$ are two parameters in the WLF equation [246]. More recently, a similar approach was used by Lu and coworkers $[269,270]$ to consider effects of moisture on the shape memory effect.

Early thermoviscoelastic models [242-245] for SMP assumed is a relatively simple process that can be described by one relaxation process (or one Maxwell or Kelvin-Viogt element in one dimensional rheological representation). However, real polymers have multiple relaxation modes, due to the generally complicated macromolecular structures, therefore requiring more branches in the constitutive model. For example, to model polymer stress-strain behavior from low to high strain rates, Mulliken and Boyce [271] used two Maxwell branches to capture the $\alpha$ - and $\beta$ - transitions, respectively, that can be excited at different strain rates. In the modeling of finite deformation behavior of amorphous polymers in and above the glass transition, Dupaix and Boyce [272] used two branches to represent intermolecular resistance and molecular network resistance. Srivastava et al. [234] used their thermo-mechanically coupled finite deformation constitutive model for polymers to study the shape memory effects [233]. There, they argued that there should M-micromechanisms that contribute to the relaxation processes in the polymer; each micromechanism can be modeled by a nonlinear Maxwell element, for which flow behavior depends on the specific micromechanism and may require a large number of material parameters. In order to reduce the number of material parameters, they chose to use only three micromechanisms, one for intermolecular interaction mechanism, and two for molecular network resistance. In the model by Srivastava and coworkers [232,233], although each micromechanism can find its own physical explanation, identifying these micromechanisms for polymers can be a challenge.

In a simplified viscoelastic representation, stress relaxation is represented by the function $\exp (-t / \tau)$, where $t$ is time and $\tau$ is the relaxation time. It easy to see that this factor starts at 1 when $t=0$, decays to 0.9 when $t=0.1 \tau$, and to $4.5 \times 10^{-5}$ when $t=10 \tau$. Therefore, $\exp (-t / \tau)$ decays from 0.9 (a noticeable change) to almost zero within two decades, too fast to be realistic for most engineering polymers, as it usually take five or more decades for a stress to be relaxed in a typical polymer. For a simple mechanical loading, this is sometimes approximately accommodated by using the so-called KWW (Kohlrausch-Williams-Watts) 
stretched exponential, i.e.,

$$
\exp \left[-\left(\frac{t}{\tau}\right)^{\beta}\right], 0<\beta \leq 1
$$

The simple exponential form is recoverd for $\beta=1$, with a smaller value of $\beta$ requirde to approximate the slower relaxation process characteristic of polymers over the time scale of interest, e.g., values for $\beta$ are often around 0.5 [273]. One disadvantage of Eq. 6 is that it is difficult to be convert into a differential form, preferred for developing constitutive models for finite element based simulations. It is known that the relaxation behavior described by Eq. 3 can also be captured by a multi-branch model (or Prony series, or generalized standard linear solid model) where multiple Maxwell elements are arranged in parallel. Therefore, it becomes a natural choice to use multi-branch models for thermoviscoelasticity. In fact, as demonstrated by Diani et al. [274] and Yu et al. [171], the multi-branch model, when being used together with the time-temperature superposition, can adequately capture the shape memory behavior. In both works, since the focuses were the shape memory behavior (instead of stress-strain behavior at high and low temperatures), linear Maxwell elements were used in individual nonequilibrium branches. For the comprehensive constitutive models for finite deformation thermomechanical behavior, Westbrook et al. [275] used one nonlinear Maxwell branch to capture the yielding behavior at temperature below glass transition temperature and multiple linear Maxwell branches for time-dependent stress-strain behavior at temperatures above glass transition; Xiao et al. [276] used multiple nonlinear nonequilibrium Maxwell elements. As shown in these models, when multiple nonlinear branches are used, the models show improved capability of capturing stress-strain behavior at different temperatures as well as shape recovery behavior in a shape memory cycle. However, the trade-off is more material parameters involved, especially when nonlinear Maxwell elements are used. In additional, the multibranch approach was shown to be effective in capture the multi-shape memory effects due to the broad temperature range of thermomechanical transition [171].

\subsection{Phase evolution models}

In semi-crystalline polymers, polymer crystallization can be used as an effective mechanism for shape memory effects. This is because the newly crystallized portion of the material (or phase) is stress-free and thus takes the current configuration as its undeformed configuration. The stress-free state of newly formed crystal phase was referred as a natural configuration by Rajagapol [277,278]. Such as concept was widely used by many researchers to study rubbers with thermally induced scissoring and reformation [279-282], and crystallizing polymers [283-286]. Since polymer crystallization is a time consuming process, the crystallization process may be coupled with mechanical deformation, such as in the recent study of two-way shape memory effect in a semi-crystalline polymer where a constant force was applied during crystallization process [196]. In this case, small polymer crystals formed a different moment have different natural (or reference) configures. Therefore, the evolution of stress-free state is also called multi-natural configuration approach [9], or phase evolution approach [287]. Such a concept was also the foundation for some of the recent constitutive models for shape memory polymers [288-291] and two-way shape memory effects [206,292]. In addition, if one takes 
"phase" as a more general representation of a small collection of material fraction that has distinct deformation history, one would find that many active materials can achieve shape memory effects or its active motion through the phase evolution mechanism, such as light activated polymers developed by Lendlein et al. [53] and Scott and coworkers [220,293], covalent adaptable network polymers with bond exchange reaction [294,295], Diels-Alder network [296,297], ionic gels [298]. Several constitutive models were developed in the recent years to model these material systems, such as light activate polymers [299-301], shape memory elastomeric composites [302] and its triple shape memory behavior [303, 304], polymers with temperature-dependent bond exchange reactions [305], and Diels-Alder networks [306]. The concept of employing phase formation to achieve shape memory effect can be easily illustrated by Fig. 22. A material in its homogeneous phase state (Fig. 22a) is stretched (Fig. 22b). While the load is held, due to environmental change or external stimulus, new phases (such as crystals) are formed (Fig. 22c). Note that these new phases are in a stress-free state. Upon unloading, however, the material cannot return to its original shape due to the requirement of force balance. Instead, it takes a new temporary shape (Fig. 22d). Upon another environmental change or external stimulus, the material recovers its original shape. Here it should be noted that the temporary shape depends the volume fraction and distribution of new phases as well as the external load applied. In addition, as it can seen from Fig. 22d, even though the sample has no external load after unloading, the material is not stress-free.

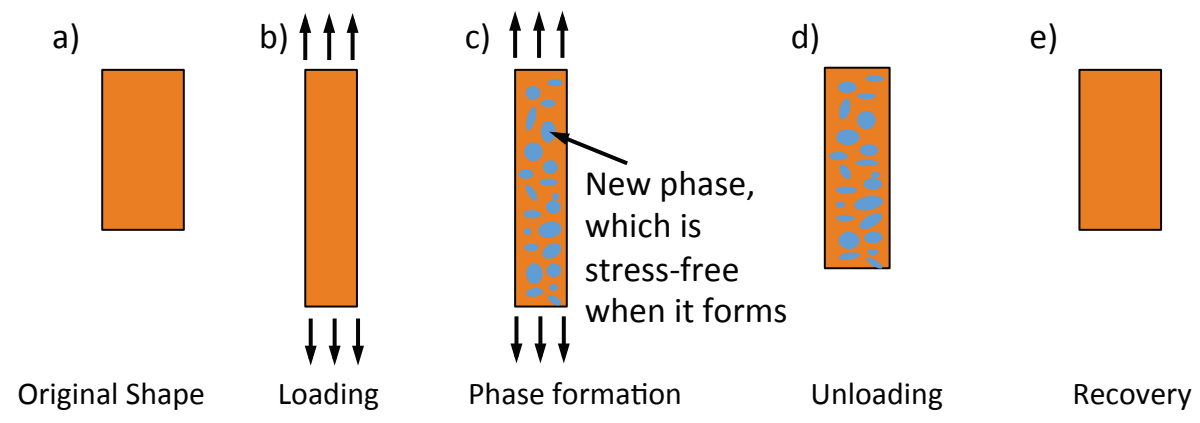

Fig. 22. Phase formation and shape memory effect.

If the phase formation and mechanical deformation occur simultaneously, the stress evolution becomes rather complicated. For example, Long et al. [305] derived the Cauchy stress tensor for an incompressible elastomer that undergoes thermally induced bond exchange reaction,

$$
\begin{gathered}
\boldsymbol{\sigma}=-\frac{\rho}{J_{t}} \mathbf{1}+\frac{C_{O R}}{J_{t} C_{0}} \Omega[I(t)] \boldsymbol{B}_{t}^{e}+\int_{0}^{t}\left[\frac{\Delta(\tau, t)}{J_{t} C_{0}} \Omega[H(\tau, t)] \boldsymbol{B}_{\tau \rightarrow t}^{e}\right] d \tau \\
\boldsymbol{B}_{\tau \rightarrow t}^{e}=\boldsymbol{F}_{\tau \rightarrow t}^{e}\left(\boldsymbol{F}_{\tau \rightarrow t}^{e}\right)^{T}, \boldsymbol{F}_{\tau \rightarrow t}^{e}=\boldsymbol{F}_{t}^{e}\left(\boldsymbol{F}_{\tau}^{e}\right)^{-1}, \\
H(\tau, t)=\operatorname{tr}\left(\boldsymbol{B}_{\tau \rightarrow t}^{e}\right), I(t)=\operatorname{tr}\left[\boldsymbol{F}_{t}^{e}\left(\boldsymbol{F}_{t}^{e}\right)^{T}\right],
\end{gathered}
$$

where $C_{O R}$ and $\Delta(\tau, t)$ are chain densities of original chains and reformed chains, $\boldsymbol{F}_{\tau \rightarrow t}^{e}$ is the mechanical deformation gradient measured from time $\tau$ to time $t, \boldsymbol{F}_{t}^{e}$ is the mechanical deformation gradient measured at time $t, \Omega(y)$ is the inverse Langevin function scaled by material parameters, $p$ is the Langrange multiplier to enforce isothermal incompressibility, $J_{t}$ 
is the total volume change. It should be noted that the integral term in Eq. 7 has the nature of convolutional integrals and therefore is computationally very expensive. A potential solution is to use a so-called effective phase to homogenize different evolving phases [286]; its 3D form, however, still needs to be developed and verified.

\subsection{Models for predicting shape memory performance}

While most of the constitutive models developed so far target capturing the comprehensive thermomechanical behavior, they are also very complicated and not so easy to be used. In recognition of this drawback, there are some modeling efforts that targets providing simple methods to predict shape memory behavior of a polymer. Diani et al. [274] used Prony series to predict the shape memory behavior and achieved excellent agreement. To predict the shape recovery time, Bonner et al. [307] proposed to use the recovery half life (similar to relaxation time). Since each recovery half life time recovers $50 \%$ of the remaining deformation, seven times of recovery half life time give $99.2 \%$ of shape recovery. For SMPs deformed and programmed at different temperatures, they found that the recovery half time can be calculated as the ratio between the viscosity measured at the recovery temperature and the initial modulus at the deformation temperature. Under the testing conditions in their paper, good agreement was achieved. Since it is well known that shape recovery of a SMP depends strongly on the recovery temperature, Ge et al. [308] developed an analytical approach to predict shape recovery. By using the KWW stretch exponential to describe the viscoelastic behavior of the SMP, for a temperature above $T_{r}$, they found that the recovery ratio $\zeta$ as a function of time can be given by

$$
\ln \zeta=-\frac{\beta}{\tau_{0}^{\beta}\left(1+\frac{E_{1}}{E_{0}}\right)}\left\{\int_{0}^{\frac{T_{r}-T_{L}}{Q}} \exp \left[\frac{A F_{c}}{k_{b}}\left(\frac{1}{T_{L}+Q t}-\frac{1}{T_{r}}\right)\right]+\int_{\frac{T_{r}-T_{L}}{Q}}^{t} 10^{\frac{C_{1}\left(T_{L}+Q t-T_{r}\right)}{C_{2}+\left(T_{L}+Q t-T_{r}\right)}}\right\} t^{\beta-1} d t,
$$

where $E_{0}$ and $E_{1}$ are the equilibrium and nonequilibrium moduli, respectively, $\beta$ is the parameter in KWW exponential, $Q$ is the heating rate, $\tau_{0}$ is the relaxation time measured at a reference temperature $T_{r}, k_{b}$ is Boltzmann's constant, $A F_{c}, C_{1}$, and $C_{2}$ are the parameters in time-temperature superposition principle. The advantage of this solution is that all the parameters in the model can be measured by a few simple rheological experiments, such as DMA tests and stress relaxation tests. However, this model cannot consider the effects of programming temperature on the recovery, that can significantly affect the recovery behavior [229-231,309,310].More recently, Yu et al. [311] systematically studied the factors affecting shape stability and shape recovery rate. They found that shape stability is uniquely determined by the relaxation modulus before unloading. According to this observation, a longer holding time after loading can give higher shape stability, but loading at a lower temperature will give lower shape stability. In addition, the recovery is determined by the shape stability and lower shape stability normally gives a fast recovery. Finally, the shape memory behavior under different themomechanical loading conditions can be unified by using the reduced time. For example, by using reduced, a master recovery curve can be constructed by using the similar concept of time-temperature superposition. 


\section{Summary and outlook}

In this review, we presented notable recent progress in the field of SMPs. Although conventional SMEs were briefly introduced, the main emphasis was placed on new enabling mechanisms such as molecular switches, new shape memory behavior (multi-SME and 2W-SME), and unconventional SMP fabrication methods. Molecular enabling mechanisms were presented from the standpoint of underlying polymer physics. Various modeling methods, providing further understanding from a different angle (i.e., solid mechanics), were also reviewed. On closing, we hereby go back to a set of questions, challenges, and opportunities that we laid out in a review [4] published in 2011:

1. "2W-SME. Can the phenomenon be extended beyond crosslinked semi-crystalline polymers? Besides reversible bending, can a $2 \mathrm{~W}-\mathrm{SME}$ be realized that allows reversible shape switching in a non-linear fashion under zero external stress? Shape memory alloys can be trained to exhibit two-way reversible surface topographic changes. Can this be realized with polymers?"

2. Multi-SME. Can we as a research community come up with the new and exciting application concepts that best utilize the versatile multi-SME?

3. "Recovery stress. Can an SMP be developed that exhibits recovery stress comparable to shape memory alloys, without compromising shape fixing and recovery behavior?"

Looking back at these questions, clearly the biggest advance and exciting surprise within a short three years has been the discovery of the true $2 \mathrm{~W}$-SME for semi-crystalline polymers. Intriguingly, recent study has shown that polymer crystals formed via strain-induced crystallization can be manipulated to precisely tune the melting temperature [312-314]. This may open up new ways to induce $2 \mathrm{~W}$-SME. Although the $2 \mathrm{~W}-\mathrm{SME}$ has only been demonstrated for semi-crystalline polymers, indications that this can be extended to other types of polymers were also reported such as the quasi $2 \mathrm{~W}-\mathrm{SME}$ for the ionomeric Nafion [315]. As for the multi-SME, materials exhibiting such a behavior have expanded significantly and molecular (instead of phase) multi-switching mechanisms have also been demonstrated, yet concepts on new applications have not advanced much. Not such exciting development has been reported in terms of the recovery stress. This is perhaps due to the lack of sufficient interest. The SMP community has increasingly realized that SMPs as soft materials would be best used in ways that are different from shape memory alloys. Thus, an ultra-high recovery stress may not be urgently needed.

Other notable advances include the enrichment of molecular switching mechanisms, that have naturally led to novel non-thermal based triggering methods that may be critical for some end applications. The various application concepts based on the surface SME are particularly noteworthy. Some of these concepts have the potential to become commercialized in the near term. Also on SMP applications, a notable development at the bulk device level is the use of SMPs as substrates for electronic components, leading to electronics with complex three dimensional shapes that are otherwise difficult to fabricate without using the shape memory properties [316-319]. Mechanical design is very important to achieve versatile shape morphing. On this front, the unconventional SMP fabrication methods summarized in this review have 
been valuable additions. With all the exciting progress within such a short time, we anticipate the SMP research to continue on its explosive pace in the near future.

\section{Acknowledgment}

TX thanks the financial support from the following programs: the National Key Basic Research Program of China (Grant No. 2015CB351903), National Natural Science Foundation of China (Grant No. 21474084); the Chinese central government's Recruitment Program of Global Experts; and the 985 program for the startup funding. QZ acknowledges the support from the State Key Laboratory of Chemical Engineering (No. SKL-ChE-14D04) and Fundamental Research Funding of Zhejiang University (No. 2014QNA4021). HJQ acknowledges support from US the Air Force Office of Scientific Research (FA9550-13-1-088, Dr. B.-L. "Les" Lee, Program Manager), US National Science Foundation (CMMI-1404621, CMMI-1404627, EFRI-1435452, EFRI-1038305). 


\section{References}

[1] Liu F, Urban MW. Recent advances and challenges in designing stimuli-responsive polymers. Prog Polym Sci 2010;35:3-23.

[2] Qiu Y, Park K. Environment-sensitive hydrogels for drug delivery. Adv Drug Deliv Rev 2001;53:321-39.

[3] Yang Z, Herd GA, Clarke SM, Tajbakhsh AR, Terentjev EM, Huck WTS. Thermal and UV shape shifting of surface topography. J Am Chem Soc 2006;128:1074-5.

[4] Xie T. Recent advances in polymer shape memory. Polymer 2011;52:4985-5000.

[5] Lendlein A, Kelch S. Shape-memory polymers. Angew Chem Int Ed 2002;41:2034 -57.

[6] Behl M, Zotzmann J, Lendlein A. Shape-memory polymers and shape changing polymers. Adv Polym Sci 2010;226:1-40.

[7] Liu C, Qin H, Mather PT. Review of progress in shape-memory polymers. J Mater Chem 2007;17:1543-58.

[8] Mather PT, Luo XF, Rousseau IA. Shape memory polymer research. Annu Rev Mater Res 2009;39:445-71.

[9] Hu JL, Zhu Y, Huang HH, Lu J. Recent advances in shape-memory polymers: Structure, mechanism, functionality, modeling and applications. Prog Polym Sci 2012;37:1720-63.

[10] Meng H, Mohamadian H, Stubblefield M, Jerro D, Ibekwe S, Pang SS, Li GQ. Various shape memory effects of stimuli-responsive shape memory polymers. Smart Mater Struct 2013;22:093001/1-23

[11] Lendlein A, Behl M, Hiebl B, Wischke C. Shape-memory polymers as a technology platform for biomedical applications. Expert Rev Med Devices 2010;7:357-79.

[12] El Feninat F, Laroche G, Fiset M, Mantovani D. Shape memory materials for biomedical applications. Adv Eng Mater 2002;4:91-104.

[13] Safranski DL, Smith KE, Gall K. Mechanical requirements of shape-memory polymers in biomedical devices. Polym Rev 2013;53:76-91. 
[14] Huang WM, Song CL, Fu YQ, Wang CC, Zhao Y, Purnawali H, Lu HB, Tang C, Ding Z, Zhang JL. Shaping tissue with shape memory materials. Adv Drug Deliv Rev $2013 ; 65: 515-35$.

[15] Wischke C, Lendlein A. Shape-memory polymers as drug carriers-a multifunctional system. Pharm Res 2010;27:527-9.

[16] Kuder IK, Arrieta AF, Raither WE, Ermanni P. Variable stiffness material and structural concepts for morphing applications. Prog Aerosp Sci 2013;63:33-55.

[17] Liu YJ, Du HY, Liu LW, Leng JS. Shape memory polymers and their composites in aerospace applications: a review. Smart Mater Struct 2014;23:023001/1-22.

[18] Zhang L, Du HY, Liu LW, Liu YJ, Leng JS. Analysis and design of smart mandrels using shape memory polymers. Composites Part B 2014;59:230-7.

[19] Weiss RA, Izzo E, Mandelbaum S. New design of shape memory polymers: Mixtures of an elastomeric ionomer and low molar mass fatty acids and their salts. Macromolecules 2008;41:2978-80.

[20] Behl M, Razzaq MY, Lendlein A. Multifunctional shape-memory polymers. Adv Mater 2010;22:3388-410.

[21] Leng JS, Lan X, Liu YJ, Du SY. Shape-memory polymers and their composites: stimulus methods and applications. Prog Mater Sci 2011;56:1077-135.

[22] Hsu L, Weder C, Rowan SJ. Stimuli-responsive, mechanically-adaptive polymer nanocomposites. J Mater Chem 2011;21:2812-22.

[23] Ratna D, Karger-Kocsis J. Recent advances in shape memory polymers and composites: a review. J Mater Sci 2008;43:254-269.

[24] Meng QH, Hu JL. A review of shape memory polymer composites and blends. Composites Part A 2009;40:1661-72.

[25] Liu YJ, Lv HB, Lan X, Leng JS, Du SY. Review of electro-active shape memory polymer composite. Compos Sci Technol 2009;69:2064-8.

[26] Huang WM, Yang B, Zhao Y, Ding Z. Thermo-moisture responsive polyurethane shape-memory polymer and composites: a review. J Mater Chem 2010;20:3367-81. 
[27] Hearon K, Singhal P, Horn J, Small W, Olsovsky C, Maitland KC, Wilson TS, Maitland DJ. Porous Shape-Memory Polymers. Polym Rev 2013;53:41-75.

[28] $\mathrm{Hu}$ JL, Lu J, Zhu Y. New developments in elastic fibers. Polym Rev 2008;48:275-301.

[29] Kotek R. Recent advances in polymer fibers. Polym Rev 2008;48:221-9.

[30] Hu JL, Chen SJ. A review of actively moving polymers in textile applications. J Mate Chem 2010;20:3346-55.

[31] Wagermaier W, Kratz K, Heuchel M, Lendlein A. Characterization methods for shape-memory polymers. Adv Polym Sci 2010;226:97-145.

[32] Xie T, Rousseau IA. Facile tailoring of thermal transition temperatures of epoxy shape memory polymers. Polymer 2009;50:1852-6.

[33] Behl M, Kratz K, Zotamann J, Nöchel U, Lendlein A. Reversible bidirectional shape-memory polymers. Adv Mater 2013;25:4466-9.

[34] Wu JJ, Lin YT, Sun JZ. Anisotropic volume change of poly(N-isopropylacrylamide)-based hydrogels with an aligned dual-network microstructure. J Mater Chem 2012;22:17449-51.

[35] Kim J, Hanna JA, Byun M, Santangelo CD, Hayward RC. Designing responsive buckled surfaces by halftone gel lithography. Science 2012;335:1201-5.

[36] Ma CX, Li TF, Zhao Q, Yang XX, Wu JJ, Luo YW, Xie T. Supramolecular Lego assembly towards three-dimensional multi-responsive hydrogels. Adv Mater 2014;26:5565-9.

[37] Zhou SB, Zheng XT, Yu XJ, Wang JX, Weng J, Li XH, Feng B, Yin M. Hydrogen bonding interaction of poly(D,L-lactide)/hydroxyapatite nanocomposites. Chem Mater 2007;19:247-53.

[38] Dong J, Weiss RA. Shape memory behavior of zinc oleate-filled elastomeric ionomers. Macromolecules 2011;4:8871-9.

[39] Shi Y, Yoonessi M, Weiss RA. High temperature shape memory polymers. Macromolecules 2013;46:4160-7.

[40] Dolog R, Weiss RA. Shape memory behavior of a polyethylene-based carboxylate ionomer. Macromolecules 2013;46:7845-52. 
[41] Gu XZ, Mather PT. Entanglement-based shape memory polyurethanes: synthesis and characterization. Polymer 2012;53:5924-34.

[42] Agarwal P, Chopra M, Archer LA. Nanoparticle netpoints for shape-memory polymers. Angew Chem Int Ed 2011;50:8670-3.

[43] Zhang YM, Wang QH, Wang C, Wang TM. High-strain shape memory polymer networks crosslinked by SiO2. J Mater Chem 2011;21:9073-8.

[44] Du K, Gan ZH. Shape memory behaviour of HA-g-PDLLA nanocomposites prepared via in situ polymerization. J Mater Chem B 2014;2:3340-8.

[45] Ishida K, Yoshie N. Two-way conversion between hard and soft properties of semicrystalline cross-linked polymer. Macromolecules 2008;41:4753-7.

[46] Inoue K, Yamashiro M, Iji M. Recyclable shape-memory polymer: poly(lactic acid) crosslinked by a thermoreversible Diels-Alder reaction. J Appl Polym Sci 2009; 112:876-85.

[47] Defize T, Riva R, Raquez JM, Dubois P, Jerome C, Alexandre M. Thermoreversibly crosslinked poly(e-caprolactone) as recyclable shape-memory polymer network. Macromol Rapid Commun 2011;32:1264-9.

[48] Rousseau IA, Xie T. Shape memory epoxy: composition, structure, properties and shape memory performances. J Mater Chem 2010;20:3431-41.

[49] Yakacki CM, Shandas R, Safranski D, Ortega AM, Sassaman K, Gall K. Strong, Tailored, biocompatible shape-memory polymer networks. Adv Funct Mater 2008; 18:2428-35.

[50] Voit W, Ware T, Dasari RR, Smith P, Danz L, Simon D, Barlow S, Marder SR, Gall K. High-strain shape-memory polymers. Adv Funct Mater 2010;20:167-71.

[51] Li JJ, Rodgers WR, Xie T. Semi-crystalline two-way shape memory elastomer. Polymer 2011;52:5320-5.

[52] Huang WM, Yang B, An L, Li C, Chan YS. Water-driven programmable polyurethane shape memory polymer: demonstration and mechanism. Appl Phys Lett 2005;86:114105/1-3. 
[53] Lendlein A, Jiang HY, Junger O, Langer R. Light-induced shape-memory polymers. Nature 2005;434:879-82.

[54] Wu LB, Jin CL, Sun XY. Synthesis, properties, and light-induced shape memory effect of multiblock polyesterurethanes containing biodegradable segments and pendant cinnamamide groups. Biomacromolecules 2011;12:235-41.

[55] Rochette JM, Ashby VS. Photoresponsive polyesters for tailorable shape memory biomaterials. Macromolecules 2013;46:2134-40.

[56] Zhao XH, Dang Y, Deng JG, Zhang JH. Photoinduced shape fixity and thermal-induced shape recovery properties based on polyvinyl alcohol bearing coumarin. Colloid Polym Sci 2014;292:85-95.

[57] Lee KM, Wang DH, Koerner H, Vaia RA, Tan LS, White TJ. Enhancement of photogenerated mechanical force in azobenzene-functionalized polyimides. Angew Chem Int Ed 2012;51:4117-21.

[58] Lee KM, Tabiryan NV, Bunning TJ, White TJ. Photomechanical mechanism and structure-property considerations in the generation of photomechanical work in glassy, azobenzene liquid crystal polymer networks. J Mater Chem 2012;22:691-8.

[59] Lee KM, Koerner H, Vaia RA, Bunning TJ, White TJ. Light-activated shape memory of glassy, azobenzene liquid crystalline polymer networks. Soft Matter 2011;7:4318-24.

[60] Xie T, Xiao XC, Li JJ, Wang RM. Encoding localized strain history through wrinkle based structural colors. Adv Mater 2010;22:4390-4.

[61] Li JJ, An YH, Huang R, Jiang HQ, Xie T. Unique aspects of a shape memory polymer as the substrate for surface wrinkling. ACS Appl Mater Interfaces 2012;4:598-603.

[62] Reddy S, Arzt E, del Campo A. Bioinspired surfaces with switchable adhesion. Adv Mater 2007;19:3833-7.

[63] Xie T, Xiao XC. Self-peeling reversible dry adhesive system. Chem Mater 2008;20:2866-8.

[64] Kim S, Sitti M, Xie T, Xiao XC. Reversible dry micro-fibrillar adhesives with thermally controllable adhesion. Soft Matter 2009;5:3689-93. 
[65] Wang RM, Xiao XC, Xie T. Viscoelastic behavior and force nature of thermo-reversible dry adhesives. Macromol Rapid Commun 2010;31:295-9.

[66] Wang RM, Xie T. Shape memory- and hydrogen bonding- based strong reversible adhesives. Langmuir 2010;26:2999-3002.

[67] Chen CM, Chiang CL, Lai CL, Xie T, Yang S. Buckling-based strong dry adhesives via interlocking. Adv Funct Mater 2013;23:3813-23.

[68] Eisenhaure J, Xie T, Varghess S, Kim S. Microstructured shape memory polymer surfaces with reversible dry adhesion. ACS Appl Mater Interfaces 2013;5:7714-7.

[69] Xu H, Yu C, Wang S, Malyarchuk V, Xie T, Rogers J. Deformable, programmable, and shape-memorizing micro-Optics. Adv Funct Mater 2013;23:3299-306.

[70] Lehn JM. Dynamers: dynamic molecular and supramolecular polymers. Prog Polym Sci $2005 ; 30: 814-31$.

[71] Maeda T, Otsuka H, Takahara A. Dynamic covalent polymers: reorganizable polymers with dynamic covalent bonds. Prog Polym Sci 2009;34:581-604.

[72] Kloxin CJ, Scott TF, Adzima BJ, Bowman CN. Covalent adaptable networks (CANs): a unique paradigm in cross-linked polymers. Macromolecules 2010;43:2643-53.

[73] Wojtecki RJ, Meador MA, Rowan SJ. Using the dynamic bond to access macroscopically responsive structurally dynamic polymers. Nat Mater 2011;10:14-27.

[74] Yang Y, Urban MW. Self-healing polymeric materials. Chem Soc Rev 2013;42:7446-67.

[75] Aoki D, Teramoto Y, Nishio Y. SH-containing cellulose acetate derivatives: preparation and characterization as a shape memory recovery material. Biomacromolecules 2007;8:3749-57.

[76] Yamauchi K, Lizotte JR, Long TE. Thermoreversible poly(alkyl acrylates) consisting of self-complementary multiple hydrogen bonding. Macromolecules 2003;36:1083-8.

[77] Li JH, Viveros JA, Wrue MH, Anthamatten M. Shape-memory effects in polymer networks containing reversibly associating side-groups. Adv Mater 2007;19:2851-5.

[78] Li JH, Lewis CL, Chen DL, Anthamatten M. Dynamic mechanical behavior of photo-cross-linked shape-memory elastomers. Macromolecules 2011;44:5336-43. 
[79] Zhu Y, Hu JL, Liu YJ. Shape memory effect of thermoplastic segmented polyurethanes with self-complementary quadruple hydrogen bonding in soft segments. Eur Phys J E 2009;28:3-10.

[80] Kushner AM, Vossler JD, Williams GA, Guan ZB. A biomimetic modular polymer with tough and adaptive properties. J Am Chem Soc 2009;131:8766-8.

[81] Kumpfer JR, Rowan SJ. Thermo-, photo-, and chemo-responsive shape-memory properties from photo-cross-linked metallo-supramolecular polymers. J Am Chem Soc 2011;133:12866-74.

[82] Capadona JR, Shanmuganathan K, Tyler DJ, Rowan SJ, Weder C. Stimuli-responsive polymer nanocomposites inspired by thesea cucumber dermis. Science 2008;319:1370-4.

[83] Mendez J, Annamalai PK, Eichhorn SJ, Rusli R, Rowan SJ, Foster EJ, Weder C. Bioinspired mechanically adaptive polymer nanocomposites with water-activated shape-memory effect. Macromolecules 2011;44:6827-6835.

[84] Koerner H, Price G, Pearce NA, Alexander M, Vaia RA. Remotely actuated polymer nanocomposites-stress-recovery of carbon-nanotube-filled thermoplastic elastomers. Nat Mater 2004;3:115-20.

[85] Fei GX, Li G, Wu LS, Xia HS. A spatially and temporally controlled shape memory process for electrically conductive polymer-carbon nanotube composites. Soft Matter 2012;8:5123-6.

[86] Mohr R, Kratz K, Weigel T, Lucka-Gabor M, Moneke M, Lendlein A. Initiation of shape-memory effect by inductive heating of magnetic nanoparticles in thermoplastic polymers. Proc Natl Acad Sci USA 2006;103:3540-5.

[87] Leng JS, Lv HB, Liu YJ, Du SY. Electroactivate shape-memory polymer filled with nanocarbon particles and short carbon fibers. Appl Phys Lett 2007;91:144105/1-3.

[88] Leng JS, Lan X, Liu YJ, Du SY, Huang WM, Liu N, Phee SJ, Yuan Q. Electrical conductivity of thermoresponsive shape-memory polymer with embedded micron sized Ni powder chains. Appl Phys Lett 2007;92:014104/1-3.

[89] Luo X, Mather PT. Conductive shape memory nanocomposites for high speed electrical actuation. Soft Matter 2010;6:2146-9. 
[90] Zhang HJ, Xia HS, Zhao Y. Optically triggered and spatially controllable shape-memory polymer-gold nanoparticle composite materials. J Mater Chem 2012;22:845-9.

[91] Zhang HJ, Zhao Y. Polymers with dual light-triggered functions of shape memory and healing using gold nanoparticles. ACS Appl Mater Interfaces 2013;5:13069-75.

[92] Zhang HJ, Zhang JM, Tong X, Ma DL, Zhao Y. Light polarization-controlled shape-memory polymer/gold nanorod composite. Macromol Rapid Commun $2013 ; 34: 1575-9$.

[93] Kohlmeyer RR, Lor M, Chen J. Remote, local, and chemical programming of healable multishape memory polymer nanocomposites. Nano Lett 2012;12:2757-62.

[94] Kohlmeyer RR, Chen J. Wavelength-selective, IR light-driven hinges based on liquid crystalline elastomer composites. Angew Chem Int Ed 2013;52:9234-7.

[95] Li G, Fei GX, Xia HS, Han JJ, Zhao Y. Spatial and temporal control of shape memory polymers and simultaneous drug release using high intensity focused ultrasound. $\mathrm{J}$ Mater Chem 2012;22:7692-6.

[96] Kumar UN, Kratz K, Heuchel M, Behl M, Lendlein A. Shape-memory nanocomposites with magnetically adjustable apparent switching temperatures. Adv Mater 2011;23:4157-62.

[97] Liu Y, Li Y, Chen HM, Yang G, Zheng XT, Zhou SB. Water-induced shape-memory poly(D,L-lactide)/microcrystalline cellulose composites. Carbohydr Polym 2014;104:101-8.

[98] Qi XD, Yao XL, Deng S, Zhou TN, Fu Q. Water-induced shape memory effect of grapheme oxide reinforced polyvinyl alcohol nanocomposites. J Mater Chem A 2014;2:2240-9.

[99] Jung YC, So HH, Cho JW. Water-responsive shape memory polyurethane block copolymer modified with polyhedral oligomeric silsesquioxane. J Macromol Sci Phys B 2006;45:453-61.

[100] Gu XZ, Mather PT. Water-triggered shape memory of multiblock thermoplastic polyurethanes (TPUs). RSC Adv 2013;3:15783-91. 
[101] Lv HB, Leng JS, Liu YJ, Du SY. Shape-memory polymer in response to solution. Adv Eng Mater 2008;10:592-5.

[102] Zhao Y, Wang CC, Huang WM, Purnawali H. Buckling of poly(methyl methacrylate) in stimulus-responsive shape recovery. Appl Phys Lett 2011;99:131911/1-3.

[103] Zhang XZ, Zhou QQ, Liu HR, Liu HW. UV light induced plasticization and light activated shape memory of spiropyran doped ethylene-vinyl acetate copolymers. Soft Matter 2014;10:3748-54.

[104] Nelson BA, King WP, Gall K. Shape recovery of nanoscale imprints in a thermoset "shape memory" polymer. Appl Phys Lett 2005;86:103108/1-3.

[105] Altebaeumer T, Gotsmann B, Pozidis H, Knoll A, Duerig U. Nanoscale shape-memory function in highly cross-linked polymers. Nano Lett 2008;8:4398-403.

[106] Wang Z, Hansen C, Ge Q, Maruf SH, Ahn DU, Qi HJ, Ding YF. Programmable, pattern-memorizing polymer surface. Adv Mater 2011;23:3669-73.

[107] Lee MH, Huntington MD, Zhou W, Yang JC, Odom TW. Programmable soft lithography: solvent-assisted nanoscale embossing. Nano Lett 2011;11:311-5.

[108] Yabu H, Jia RK, Matsuo Y, Ijiro K, Yamamoto S, Nishino F, Takaki T, Kuwahara M, Shimomura M. Preparation of highly oriented nano-pit arrays by thermal shrinking of honeycomb-patterned polymer films. Adv Mater 2008;20:4200-4.

[109] Carlson A, Bowen AM, Huang YG, Nuzzo RG, Rogers JA. Transfer printing techniques for materials assembly and micro/nanodevice fabrication. Adv Mater 2012;24:5284-318.

[110] Pretsch T, Ecker M, Schildhauer M, Maskos M. Switchable information carriers based on shape memory polymer. J Mater Chem 2012;22:7757-66.

[111] Ebara M, Uto K, Idota N, Hoffman JM, Aoyagi T. Shape-memory surface with dynamically tunable nanogeometry activated by body heat. Adv Mater 2012;24:273-8.

[112] Davis KA, Burke KA, Mather PT, Henderson JH. Dynamic cell behavior on shape memory polymer substrates. Biomaterials 2011;32:2285-93.

[113] Tseng LF, Mather PT, Henderson JH. Shape-memory-actuated change in scaffold fiber alignment directs stem cell morphology. Acta Biomater 2013;9:8790-801. 
[114] Li WB, Gong T, Chen HM, Wang L, Li JR, Zhou SB. Tuning surface micropattern features using a shape memory functional polymer. RSC Adv 2013;3:9865-74.

[115] Chen CM, Yang S. Directed water shedding on high-aspect-ratio shape memory polymer micropillar arrays. Adv Mater 2014;26:1283-8.

[116] Fei GX, Tuinea-Bobe G, Li DX, Li G, Whiteside B, Coates P, Xia HS. Electro-activated surface micropattern tuning for microinjection molded electrically conductive shape memory polyurethane composites. RSC Adv 2013;3:24132-9.

[117] Drury JL, Mooney DJ. Hydrogels for tissue engineering: scaffold design variables and applications. Biomaterials 2003;24:4337-51.

[118] Chung BG, Lee KH, Khademhosseini A, Lee SH. Microfluidic fabrication of microengineered hydrogels and their application in tissue engineering. Lab Chip 2012;12:45-59.

[119] Osada Y, Matsuda A. Shape memory in hydrogels. Nature 1995;376:219.

[120] Kagami Y, Gong JP, Osada Y. Shape memory behaviors of crosslinked copolymers containing stearyl acrylate. Macromol Rapid Commun 1996;17:539-43.

[121] Merline JD, Nair CPR, Gouri C, Shrisudha T, Ninan KN. Shape memory characterization of polytetra methylene oxide/poly (acrylic acid-co-acrylonitrile) complexed gel. J Mater Sci 2007;42:5897-902.

[122] Izawa H, Kadokawa J. Preparation and characterizations of functional ionic liquid-gel and hydrogel materials of xanthan gum. J Mater Chem 2010;20:5235-41.

[123] Skrzeszewska PJ, Jong LN, de Wolf FA, Stuart MAC, van der Gucht J. Shape-memory effects in biopolymer networks with collagen-like transient nodes. Biomacromolecules 2011;12:2285-92.

[124] Hao JK, Weiss RA. Mechanically tough, thermally activated shape memory hydrogels. ACS Macro Lett 2013;2:86-9.

[125] Inomata K, Terahama T, Sekoguchi R, Sugimoto H, Nakanishi E. Shape memory properties of polypeptide hydrogels having hydrophobic alkyl side chains. Polymer $2012 ; 53: 3281-6$. 
[126] Wang CC, Huang WM, Ding Z, Zhao Y, Purnawali H. Cooling-/water-responsive shape memory hybrids. Compos Sci Technol 2012;72:1178-82.

[127] Yasin A, Li HZ, Lu Z, Rehman S, Siddiq M, Yang HY. A shape memory hydrogel induced by the interactions between metal ions and phosphate. Soft Matter 2014;10:972-7.

[128] Harris RD, Auletta JT, Motlagh SAM, Lawless MJ, Perri NM, Saxena S, Weiland LM, Waldeck DH, Clark WW, Meyer TY. Chemical and electrochemical manipulation of mechanical properties in stimuli-responsive copper-cross-linked hydrogels. ACS Macro Lett 2013;2:1095-9.

[129] Sangeetha NM, Maitra U. Supramolecular gels: functions and uses. Chem Soc Rev 2005;34:821-36.

[130] Yan XZ, Wang F, Zheng B, Huang FH. Stimuli-responsive supramolecular polymeric materials. Chem Soc Rev 2012;41:6042-65.

[131] Han XJ, Dong ZQ, Fan MM, Liu Y, Li JH, Wang YF, Yuan QJ, Li BJ, Zhang S. pH-Induced shape-memory polymers. Macromol Rapid Commun 2012;33:1055-60.

[132] Dong ZQ, Cao Y, Yuan QJ, Wang YF, Li JH, Li BJ, Zhang S. Redox- and glucose-induced shape-memory polymers. Macromol Rapid Commun 2013;34:867-72.

[133] Peters O, Ritter H. Supramolecular controlled water uptake of macroscopic materials by a cyclodextrin-induced hydrophobic-to-hydrophilic transition. Angew Chem Int Ed $2013 ; 52: 8961-3$.

[134] Myung D, Koh WU, Ko JM, Hu Y, Carrasco M, Noolandi J, Ta CN, Frank CW. Biomimetic strain hardening in interpenetrating polymer network hydrogels. Polymer 2007;48:5376-87.

[135] Miyata T, Asami N, Uragami T. A reversibly antigen-responsive hydrogel. Nature 1999;399:766-9.

[136] Xu J, Wong DHC, Byrne JD, Chen K, Bowerman C, DeSimone JM. Future of the particle replication in nonwetting templates (PRINT) technology. Angew Chem Int Ed $2013 ; 52: 6580-9$. 
[137] Marshall JE, Gallagher S, Terentjev EM, Smoukov SK. Anisotropic colloidal micromuscles from liquid crystal elastomers. J Am Chem Soc 2014;136:474-9.

[138] Saha S, Copic D, Bhaskar S, Clay N, Donini A, Hart AJ, Lahann J. Chemically controlled bending of compositionally anisotropic microcylinders. Angew Chem Int Ed 2012;51:660-5.

[139] Wischke C, Schossig M, Lendlein A. Shape-memory effect of micro-/nanoparticles from thermoplastic multiblock copolymers. Small 2014;10:83-7.

[140] Wischke C, Lendlein A. Method for preparation, programming, and characterization of miniaturized particulate shape-memory polymer matrices. Langmuir 2014;30:2820-7.

[141] Bellin I, Kelch S, Langer R, Lendlein A. Polymeric triple-shape materials. Proc Natl Acad Sci USA 2006;103:18043-7.

[142] Behl M, Lendlein A. Triple-shape polymers. J Mater Chem 2010;20:3335-45.

[143] Zhao Q, Behl M, Lendlein A. Shape-memory polymers with multiple transitions: complex actively moving polymers. Soft Matter, 2013;9:1744-55.

[144] Liu GQ, Ding XB, Cao YP, Zheng ZH, Peng YX. Novel shape-memory polymer with two transition temperatures. Macromol Rapid Commun 2005;26:649-52.

[145] Luo XF, Mather PT. Triple-shape polymeric composites (TSPCs). Adv Funct Mater 2010;20:2649-56.

[146] Xie T, Xiao XC, Cheng YT. Revealing triple-shape memory effect by polymer bilayers. Macromol Rapid Commun 2009;30:1823-7.

[147] Qin H, Mather P. Combined one-way and two-way shape memory in a glass-forming nematic network. Macromolecules 2009;42:273-80.

[148] Ahn SK, Deshmukh P, Kasi RM. Shape memory behavior of side-chain liquid crystalline polymer networks triggered by dual transition temperatures. Macromolecules 2010;43:7330-40.

[149] Ahn SK, Kasi RM. Exploiting microphase-separated morphologies of side-chain liquid crystalline polymer networks for triple shape memory properties. Adv Funct Mater 2011;21:4543-9. 
[150] Suchao-in K, Chirachanchai S. "Grafting to" as a novel and simple approach for triple-shape memory polymers. ACS Appl Mater Interfaces 2013;5:6850-3.

[151] Xiao LP, Wei M, Zhan MQ, Zhang JJ, Xie H, Deng XY, Yang KK, Wang YZ. Novel triple-shape PCU/PPDO interpenetrating polymer networks constructed by selfcomplementary quadruple hydrogen bonding and covalent bonding. Polym Chem $2014 ; 5: 2231-41$.

[152] Kolesov IS, Radusch HJ. Multiple shape-memory behavior and thermal-mechanical properties of peroxide cross-linked blends of linear and short-chain branched polyethylenes. Express Polym Lett 2008;2:461-73.

[153] Hoeher R, Raidt T, Krumm C, Meuris M, Katzenberg F, Tiller JC. Tunable multiple-shape memory polyethylene blends. Macromol Chem Phys 2013;214:2725-32.

[154] Chen HM, Liu Y, Gong T, Wang L, Zhao KQ, Zhou SB. Use of intermolecular hydrogen bonding to synthesize triple-shape memory supermolecular composites. RSC Adv 2013;3:7048-56.

[155] Chen SJ, Yuan HM, Ge ZC, Chen SG, Zhuo HT, Liu JH. Insights into liquid-crystalline shape-memory polyurethane composites based on an amorphous reversible phase and hexadecyloxybenzoic acid. J Mater Chem C 2014;2:1041-9.

[156] Bae CY, Park JH, Kim EY, Kang YS, Kim BK. Organic-inorganic nanocomposite bilayers with triple shape memory effect. J Mater Chem 2011;21:11288-95.

[157] Ware T, Hearon K, Lonnecker A, Wooley KL, Maitland DJ, Voit W. Triple-shape memory polymers based on self-complementary hydrogen bonding. Macromolecules $2012 ; 45: 1062-9$.

[158] Wang SQ, Kaneko D, Okajima M, Yasaki K, Tateyama S, Kaneko T. Hyperbranched polycoumarates with photofunctional multiple shape memory. Angew Chem Int Ed 2013;52:11143-8.

[159] Luo HS, Hu JL, Zhu Y. Polymeric shape memory nanocomposites with heterogeneous twin switches. Macromol Chem Phys 2011;212:1981-6.

[160] Han YJ, Bai T, Liu Y, Zhai XY, Liu WG. Zinc ion uniquely induced triple shape memory effect of dipole-dipole reinforced ultra-high strength hydrogels. Macromol Rapid Commun 2012;33:225-31. 
[161] Bai T, Han YJ, Zhang P, Wang W, Liu WG. Zinc ion-triggered two-way macro-/microscopic shape changing and memory effects in high strength hydrogels with pre-programmed unilateral patterned surfaces. Soft Matter 2012;8:6846-52.

[162] Nan WJ, Wang W, Gao H, Liu WG. Fabrication of a shape memory hydrogel based on imidazole-zinc ion coordination for potential cellencapsulating tubular scaffold application. Soft Matter 2013;9:132-7.

[163] Xie T. Tunable polymer multi-shape memory effect. Nature 2010;464:267-70.

[164] Miaudet P, Derré A, Maugey M, Zakri C, Piccione PM, Inoubli R, Poulin P. Shape and temperature memory of nanocomposites with broadened glass transition. Science 2007;318:1294-6.

[165] Kratz K, Madbouly SA, Wagermaier W, Lendlein A. Temperature-memory polymer networks with crystallizable controlling units. Adv Mater 2011;23:4058-62.

[166] Xie T, Page KA, Eastman SA. Strain-based temperature memory effect for nafion and its molecular origins. Adv Funct Mater 2011;21:2057-66.

[167] Kratz K, Voigt U, Lendlein A. Temperature-memory effect of copolyesterurethanes and their application potential in minimally invasive medical technologies. Adv Funct Mater 2012;22:3057-65.

[168] Razzaq MY, Behl M, Lendlein A. Magnetic memory effect of nanocomposites. Adv Funct Mater 2012;22:184-91.

[169] Wang L, Di SB, Wang WX, Chen HM, Yang XF, Gong T, Zhou SB. Tunable temperature memory effect of photo-cross-linked star PCL-PEG networks. Macromolecules 2014;47:1828-36.

[170] Sun L, Huang WM. Mechanisms of the multi-shape memory effect and temperature memory effect in shape memory polymers. Soft Matter 2010;6:4403-6.

[171] Yu K, Xie T, Leng JS, Ding YF, Qi HJ. Mechanisms of multi-shape memory effects and associated energy release in shape memory polymers. Soft Matter 2012;8:5687-95.

[172] Luo YW, Guo YL, Gao X, Li BG, Xie T. A general approach towards thermoplastic multishape-memory polymers via sequence structure design. Adv Mater 2013;25:7430-8. 
[173] Wang L, Yang XF, Chen HM, Yang G, Gong T, Li WB, Zhou SB. Multi-stimuli sensitive shape memory poly(vinyl alcohol)-graft-polyurethane. Polym Chem 2013;4:4461-8.

[174] Li J, Liu T, Xia S, Pan Y, Zheng ZH, Ding XB, Peng YX. A versatile approach to achieve quintuple-shape memory effect by semi-interpenetrating polymer networks containing broadened glass transition and crystalline segments. J Mater Chem 2011;21:12213-7.

[175] Shao Y, Lavigueur C, Zhu XX. Multishape memory effect of norbornene-based copolymers with cholic acid pendant groups. Macromolecules 2012;45:1924-30.

[176] Bai YK, Jiang C, Wang QH, Wang TM. Multi-shape-memory property study of novel poly(e-caprolactone)/ethyl cellulose polymer networks. Macromol Chem Phys 2013;214:2465-72.

[177] Zhang QL, Song SJ, Feng JC, Wu PY. A new strategy to prepare polymer composites with versatile shape memory properties. J Mater Chem 2012;22:24776-82.

[178] He ZW, Satarkar N, Xie T, Cheng YT, Hilt JZ. Remote controlled multishape polymer nanocomposites with selective radiofrequency actuations. Adv Mater 2011;23:3192-6.

[179] Liu Y, Boyles JK, Genzer J, Dickey MD. Self-folding of polymer sheets using local light absorption. Soft Matter 2012;8:1764-9.

[180] DiOrio AM, Luo XF, Lee KM, Mather PT. A functionally graded shape memory polymer. Soft Matter 2011;7:68-74.

[181] Zeng C, Seino H, Ren J, Yoshie N. Polymers with multishape memory controlled by local glass transition temperature. ACS Appl Mater Interfaces 2014;6:2753-8.

[182] Razzaq MY, Behl M, Kratz K, Lendlein A. Triple-shape effect in polymer-based composites by cleverly matching geometry of active component with heating method. Adv Mater 2013;25:5514-8.

[183] Pei ZQ, Yang Y, Chen QM, Terentjev EM, Wei Y, Ji Y. Mouldable liquid-crystalline elastomer actuators with exchangeable covalent bonds. Nat Mater 2014;13:36-41. 
[184] Zhang JJ, Niu Y, Huang CL, Xiao LP, Chen ZT, Yang KK, Wang YZ. Self-healable and recyclable triple-shape PPDO-PTMEG co-network constructed through thermoreversible Diels-Alder reaction. Polym Chem 2012;3:1390-3.

[185] de Gennes PG. Réflexions sur un type de polymères nématiques. C R Seances Acad Sci Ser B 1975;281:101-3.

[186] Finkelmann H, Kock HJ, Rehage G. Liquid crystalline elastomers - a new type of liquid crystalline material. Makromol Chem Rapid Commun 1981;2:317-22.

[187] Krause S, Zander F, Bergmann G, Brandt H, Wertmer H, Finkelmann H. Nematic main-chain elastomers: coupling and orientational behavior. C R Chim 2009;12:85-104.

[188] Broemmel F, Kramer D, Finkelmann H. Preparation of Liquid Crystalline Elastomers. Adv Polym Sci 2012;250:1-48.

[189] Ohm C, Brehmer M, Zentel R. Liquid Crystalline Elastomers as Actuators and Sensors. Adv Mater 2010;22:3366-87.

[190] Ikeda T, Mamiya J, Yu YL. Photomechanics of liquid-crystalline elastomers and other polymers. Angew Chem Int Ed 2007;46:506-28.

[191] Kupfer J, Finkelmann H. Nematic liquid single crystal elastomers. Makromol Chem Rapid Commun 1991;12:717-26.

[192] Schatzle J, Kaufhold W, Finkelmann H. Nematic elastomers: the influence of external mechanical stress on the liquid-crystalline phase behavior. Makromol Chem $1989 ; 190: 3269-84$.

[193] Kaufhold W, Finkelmann H. Nematic elastomers, 1 Effect of the spacer length on the mechanical coupling between network anisotropy and nematic order. Makromol Chem 1991;192:2555-79.

[194] Tajbakhsh AR, Terentjeva EM. Spontaneous thermal expansion of nematic elastomers. Eur Phys J E 2001;6:181-8.

[195] Ahir SV, Tajbakhsh AR, Terentjev EM. Self-assembled shape-memory fibers of triblock liquid-crystal polymers. Adv Funct Mater 2006;16:556-60.

[196] Chung T, Rorno-Uribe A, Mather PT. Two-way reversible shape memory in a semicrystalline network. Macromolecules 2008;41:184-92. 
[197] Raquez JM, Vanderstappen S, Meyer F, Verge P, Alexandre M, Thomassin JM, Jerome C, Dubois P. Design of cross-linked semicrystalline poly(epsilon-caprolactone)-based networks with one-way and two-way shape-memory properties through Diels-Alder reactions. Chem Eur J 2011;17:10135-43.

[198] Razzaq MY, Behl M, Nochel U, Lendlein A. Magnetically controlled shape-memory effects of hybrid nanocomposites from oligo(omega-pentadecalactone) and covalently integrated magnetite nanoparticles. Polymer 2014;55:5953-60

[199] Razzaq MY, Behl M, Kratz K, Lendlein A. Multifunctional hybrid nanocomposites with magnetically controlled reversible shape-memory effect. Adv Mater $2013 ; 25: 5730-3$.

[200] Baker RM, Henderson JH, Mather PT. Shape memory poly(3-caprolactone)-co-poly(ethylene glycol) foams with body temperature triggering and two-way actuation. J Mater Chem B 2013;1:4916-20.

[201] Bothe M, Pretsch T. Two-way shape changes of a shape-memory poly(ester urethane). Macromol Chem Phys 2012;213:2378-85.

[202] Bai YK, Zhang XR, Wang QH, Wang TM. A tough shape memory polymer with triple-shape memory and two-way shape memory properties. J Mater Chem A $2014 ; 2: 4771-8$

[203] Zotzmann J, Behl M, Hofmann D, Lendlein A. Reversible triple-shape effect of polymer networks containing polypentadecalactone- and poly(epsilon-caprolactone)-segments. Adv Mater 2010;22:3424-9.

[204] Chen SJ, Hu JL, Zhuo HT, Zhu Y. Two-way shape memory effect in polymer laminates. Mater Lett 2008;62:4088-90.

[205] Hu JL, Chen SJ, Zhuo HT. Properties and mechanism of two-way shape memory polyurethane composites. Compos Sci Technol 2010;70:1437-43.

[206] Westbrook KK, Mather PT, Parakh V, Dunn ML, Lee BM, Qi HJ. Two-way reversible shape memory effects in a free-standing polymer composite. Smart Mater Struct 2011;20:065010/1-9.

[207] Ge Q, Westbrook KK, Mather PT, Dunn ML, Qi HJ. Thermomechanical behavior of a two-way shape memory composite actuator. Smart Mater Struct 2013;22:055009/1-11. 
[208] Behl M, Kratz K, Nöchel U, Sauter T, Lendlein A. Temperature-memory polymer actuators. Proc Natl Acad Sci USA 2013;110:12555-9.

[209] Zhou J, Turner SA, Brosnan SM, Li QX, Carrillo JMY, Nykypanchuk D, Gang O, Ashby VS, Dobrynin AV, Sheiko SS. Shapeshifting: reversible shape memory in semicrystalline elastomers. Macromolecules 2014;47:1768-76.

[210] Turner SA, Zhou J, Sheiko SS, Ashby VS. Switchable micropatterned surface topographies mediated by reversible shape memory. ACS Appl Mater Interfaces $2014 ; 6: 8017-21$.

[211] Bothe M, Pretsch T. Bidirectional actuation of a thermoplastic polyurethane elastomer. J Mater Chem A 2013;1:14491-7.

[212] Sun B, Long YZ, Zhang HD, Li MM, Duvail JL, Jiang XY, Yin HL. Advances in three-dimensional nanofibrous macrostructures via electrospinning. Prog Polym Sci 2014;39:862-90

[213] Chen HL, Cao XY, Zhang JN, Zhang JJ, Ma YM, Shi GQ, Ke YC, Tong DW, Jiang L. Electrospun shape memory film with reversible fibrous structure. J Mater Chem 2012;22:22387-91.

[214] Luo XF, Mather PT. Preparation and characterization of shape memory elastomeric composites. Macromolecules 2009;42:7251-3.

[215] Rodriguez ED, Weed DC, Mather PT. Anisotropic shape-memory elastomeric composites: fabrication and testing. Macromol Chem Phys 2013;214:1247-57.

[216] Ahn BY, Shoji D, Hansen CJ, Hong E, Dunand DC, Lewis JA. Printed Origami Structures. Adv Mater 2010;22:2251-4.

[217] Derby B. Printing and prototyping of tissues and scaffolds. Science 2012;338:921-6.

[218] Ge Q, Qi HJ, Dunn ML. Active materials by four-dimension printing. Appl Phys Lett 2013;103:131901/1-5.

[219] Ge Q, Dunn C, Qi HJ, Dunn ML. Active Origami by 4D Printing. Smart Mater Struct 2014;23:094007/1-15.

[220] Scott TF, Schneider AD, Cook WD, Bowman CN. Photoinduced plasticity in cross-linked polymers. Science 2005;308:1615-7. 
[221] Kloxin CJ, Scott TF, Park HY, Bowman CN. Mechanophotopatterning on a photoresponsive elastomer. Adv Mater 2011;23:1977-81.

[222] Fairbanks BD, Singh SP, Bowman CN, Anseth KS. Photodegradable, photoadaptable hydrogels via radical-mediated disulfide fragmentation reaction. Macromolecules 2011;44:2444-50.

[223] Michal BT, Jaye CA, Spencer EJ, Rowan SJ. Inherently photohealable and thermal shape-memory polydisulfide networks. ACS Macro Lett 2013;2:694-9.

[224] Nair DP, Cramer NB, Gaipa JC, McBride MK, Matherly EM, McLeod RR, Shandas R, Bowman CN. Two-stage reactive polymer network forming systems. Adv Funct Mater 2012;22:1502-10.

[225] Podgórski M, Nair DP, Chatani S, Berg G, Bowman CN. Programmable mechanically assisted geometric deformations of glassy two-stage reactive polymeric materials. ACS Appl Mater Interfaces 2014;6:6111-9.

[226] Felton SM, Tolley MT, Shin BH, Onal CD, Demaine ED, Rus D, Wood RJ. Self-folding with shape memory composites. Soft Matter 2013;9:7688-94.

[227] Castro F, Westbrook KK, Long KN, Shandas R, Qi HJ. Effects of thermal rates on the thermomechanical behaviors of amorphous shape memory polymers. Mech Time-Depend Mater 2010;14:219-41.

[228] Heuchel M, Cui J, Kratz K, Kosmella H, Lendlein A. Relaxation based modeling of tunable shape recovery kinetics observed under isothermal conditions for amorphous shape-memory polymers. Polymer 2010;51:6212-8.

[229] Chen X, Nguyen TD. Influence of thermoviscoelastic properties and loading conditions on the recovery performance of shape memory polymers. Mech Mater 2011;43:127-38.

[230] McClung AJW, Tandon GP, Baur JW. Strain rate- and temperature-dependent tensile properties of an epoxy-based, thermosetting, shape memory polymer (Veriflex-E). Mech Time-Depend Mater 2012;16:205-21.

[231] McClung AJW, Tandon GP, Baur JW. Deformation rate-, hold time-, and cycle-dependent shape-memory performance of Veriflex-E resin. Mech Time-Depend Mater 2013;17:39-52. 
[232] Tobushi H, Hara H, Yamada E, Hayashi S. Thermomechanical properties in a thin film of shape memory polymer of polyurethane series. Smart Mater Struct 1996;5:483-91.

[233] Srivastava V, Chester SA, Anand L. Thermally actuated shape-memory polymers: Experiments, theory, and numerical simulations. J Mech Phys Solids 2010;58:1100-24.

[234] Srivastava V, Chester SA, Ames NM, Anand L. A thermo-mechanically-coupled large-deformation theory for amorphous polymers in a temperature range which spans their glass transition. Int J Plast 2010;26:1138-82.

[235] Nguyen TD. Modeling Shape-Memory Behavior of Polymers. Polym Rev $2013 ; 53: 130-52$.

[236] Heuchel M, Sauter T, Kratz K, Lendlein A. Thermally induced shape-memory effects in polymers: Quantification and related modeling approaches. J Polym Sci Part B Polym Phys 2013;51:621-37.

[237] Qi HJ, Dunn ML. Thermomechanical behavior and modeling approaches. In: Leng JS, Du SY, editors. Shape-Memory Polymers and Multifunctional Composites. Boca Raton FL: CRC Press, 2010. p. 65-90.

[238] Flory PJ. Principles of polymer chemistry. Ithaca: Cornell University Press, 1953. p. $432-88$.

[239] Ferry JD. Viscoelastic properties of polymers. 3d ed. New York: Wiley, 1980. p. 1-20.

[240] McKenna GB. Glass formation and glassy behavior. In: Booth C, Price C, editors. Comprehensive Polymer Science: the Synthesis, Characterization, Reactions \& Applications of Polymers. Oxford UK: Pergamon, 1989. p. 311-62.

[241] Hutchinson JM. Physical Aging of Polymers. Prog Polym Sci 1995;20:703-60.

[242] Tobushi H, Hashimoto T, Hayashi S, Yamada E. Thermomechanical constitutive modeling in shape memory polymer of polyurethane series. J Intell Mater Syst Struct 1997;8:711-8.

[243] Tobushi H, Ito N, Takata K, Hayashi S. Thermomechanical constitutive modeling of polyurethane-series shape memory polymer. Shape Mem Mater 2000;327-3:343-6.

[244] Tobushi H, Okumura K, Hayashi S, Ito N. Thermomechanical constitutive model of shape memory polymer. Mech Mater 2001;33:545-54. 
[245] Nguyen TD, Qi HJ, Castro F, Long KN. A thermoviscoelastic model for amorphous shape memory polymers: Incorporating structural and stress relaxation. J Mech Phys Solids 2008;56:2792-814.

[246] Williams ML, Landel RF, Ferry JD. Temperature Dependence of Relaxation Mechanisms in Amorphous Polymers and Other Glass-Forming Liquids. J Am Chem Soc $1955 ; 77: 3701-7$.

[247] Liu YP, Gall K, Dunn ML, Greenberg AR, Diani J. Thermomechanics of shape memory polymers: Uniaxial experiments and constitutive modeling. Int J Plast 2006;22:279-313.

[248] Diani J, Liu YP, Gall K. Finite strain 3D thermoviscoelastic constitutive model for shape memory polymers. Polym Eng Sci 2006;46:486-92.

[249] Chen YC, Lagoudas DC. A constitutive theory for shape memory polymers. Part I Large deformations. J Mech Phys Solids 2008;56:1752-65.

[250] Chen YC, Lagoudas DC. A constitutive theory for shape memory polymers. Part II - A linearized model for small deformations. J Mech Phys Solids 2008;56:1766-78.

[251] Kafka V. Shape memory polymers: A mesoscale model of the internal mechanism leading to the SM phenomena. Int J Plast 2008;24:1533-48.

[252] Bol M, Reese S. Computer simulations of temperature induced Shape Memory Polymers by means of the finite element method. In: Boukamel A, Laiarinandrasana L, Meo S, Verron E, editors. Constitutive Models for Rubber V. London: Taylor \& Francis Group, 2008. p. 93-9.

[253] Bol M, Reese S. Micromechanical modelling of shape memory polymers. Adv Sci Tech $2009 ; 54: 137-42$.

[254] Xu W, Li GQ. Constitutive modeling of shape memory polymer based self-healing syntactic foam. Int J Solids Struct 2010;47:1306-16.

[255] Baghani M, Naghdabadi R, Arghavani J, Sohrabpour S. A thermodynamically-consistent $3 \mathrm{D}$ constitutive model for shape memory polymers. Int J Plast 2012;35:13-30. 
[256] Baghani M, Naghdabadi R, Arghavani J. A large deformation framework for shape memory polymers: Constitutive modeling and finite element implementation. J Intell Mater Syst Struct 2013;24:21-32.

[257] Srinivasa AR, Gosh P. A simple, Gibbs potential based multinetwork model for shape memory polymers. Aip Conf Proc 2008;1029:58-74.

[258] Ghosh P, Srinivasa AR. A Two-network thermomechanical model of a shape memory polymer. Int J Eng Sci 2011;49:823-38.

[259] Ghosh P, Reddy JN, Srinivasa AR. Development and implementation of a beam theory model for shape memory polymers. Int J Solids Struct 2013;50:595-608.

[260] Ghosh P, Srinivasa AR. A two-network thermomechanical model and parametric study of the response of shape memory polymers. Mech Mater 2013;60:1-17.

[261] Shi GH, Yang QS, He XQ. Analysis of intelligent hinged shell structures: deployable deformation and shape memory effect. Smart Mater Struct 2013;22:125018/1-8.

[262] Shi GH, Yang QS, He XQ, Liew KM. A Three-Dimensional Constitutive Equation And Finite Element Method Implementation for Shape Memory Polymers. Cmes-Comp Model Eng 2013;90:339-58.

[263] Balogun O, Mo C. Shape memory polymers: three-dimensional isotropic modeling. Smart Mater Struct 2014;23:045008/1-7.

[264] Ghosh P, Srinivasa AR. Development of a finite strain two-network model for shape memory polymers using QR decomposition. Int J Eng Sci 2014;81:177-91.

[265] Choi J, Ortega AM, Xiao R, Yakacki CM, Nguyen TD. Effect of physical aging on the shape-memory behavior of amorphous networks. Polymer 2012;53:2453-64.

[266] Xu W, Li GQ. Thermoviscoplastic Modeling and Testing of Shape Memory Polymer Based Self-Healing Syntactic Foam Programmed at Glassy Temperature. J Appl Mech 2011;78:061017/1-14.

[267] Huang WM, Yang B, Li C, Chan YS, An L. Response to "Comment on 'Water-driven programmable polyurethane shape memory polymer: Demonstration and mechanism [Appl Phys Lett 97,056101, (2010)]"'. Appl Phys Lett 2010;97:056102/1-1. 
[268] Xiao R, Nguyen TD. Modeling the solvent-induced shape-memory behavior of glassy polymers. Soft Matter 2013;9:9455-64.

[269] Lu HB, Huang WM, Leng JS. A phenomenological model for simulating the chemo-responsive shape memory effect in polymers undergoing a permeation transition. Smart Mater Struct 2014;23:045038/1-9.

[270] Lu HB, Du SY. A phenomenological thermodynamic model for the chemo-responsive shape memory effect in polymers based on Flory-Huggins solution theory. Polym Chem 2014;5:1155-62.

[271] Mulliken AD, Boyce MC. Mechanics of the rate-dependent elastic-plastic deformation of glassy polymers from low to high strain rates. Int J Solids Struct 2006;43:1331-56.

[272] Dupaix RB, Boyce MC. Constitutive modeling of the finite strain behavior of amorphous polymers in and above the glass transition. Mech Mater 2007;39:39-52.

[273] Strobl GR. The physics of polymers: concepts for understanding their structures and behavior. Berlin/New York: Springer, 1996. p. 255-6.

[274] Diani J, Gilormini P, Fredy C, Rousseau I. Predicting thermal shape memory of crosslinked polymer networks from linear viscoelasticity. Int J Solids Struct 2012;49:793-9.

[275] Westbrook KK, Kao PH, Castro F, Ding YF, Qi HJ. A 3D finite deformation constitutive model for amorphous shape memory polymers: A multi-branch modeling approach for nonequilibrium relaxation processes. Mech Mater 2011;43:853-69.

[276] Xiao R, Choi JW, Lakhera N, Yakacki CM, Frick CP, Nguyen TD. Modeling the glass transition of amorphous networks for shape-memory behavior. J Mech Phys Solids 2013;61:1612-35.

[277] Rajagopal KR, Srinivasa AR. Mechanics of the inelastic behavior of materials - Part 1, theoretical underpinnings. Int J Plast 1998;14:945-67.

[278] Rajagopal KR, Srinivasa AR. Mechanics of the inelastic behavior of materials. Part II: Inelastic response. Int J Plast 1998;14:969-95.

[279] Rajagopal KR, Wineman AS. A Constitutive Equation for Nonlinear Solids Which Undergo Deformation Induced Microstructural Changes. Int J Plast 1992;8:385-95. 
[280] Huntley HE, Wineman AS, Rajagopal KR. Stress softening, strain localization and permanent set in the circumferential shear of an incompressible elastomeric cylinder. Ima J Appl Math 1997;59:309-38.

[281] Wineman A, Min JH. Viscoelastic hinge formation in beams. Acta Mech 2000;140:183-205.

[282] Wineman A, Min JH. Time dependent scission and cross-linking in an elastomeric cylinder undergoing circular shear and heat conduction. Int J Nonlinear Mech 2003;38:969-83.

[283] Negahban M, Wineman AS. Modeling the Mechanical Response of a Material Undergoing Continuous Isothermal Crystallization. Int J Eng Sci 1992;30:953-62.

[284] Negahban M. Simulation of Mechanical Response in Crystallizing Polymers Crystallization under Constant Shearing Deformations. Mech Mater 1993;16:379-99.

[285] Ma R, Negahban M. Simulation of Mechanical Response in Crystallizing Polymers Crystallization under a Constant Shear Force. Acta Mech 1995;112:59-76.

[286] Ma RJ, Negahban M. Simulation of Mechanical Response during Polymer Crystallization around Rigid Inclusions and Voids - Homogeneous Crystallization. Mech Mater 1995;21:25-50.

[287] Long KN, Dunn ML, Qi HJ. Mechanics of soft active materials with phase evolution. Int J Plast 2010;26:603-16.

[288] Barot G, Rao IJ. Constitutive modeling of the mechanics associated with crystallizable shape memory polymers. Z Angew Math Phys 2006;57:652-81.

[289] Barot G, Rao IJ, Rajagopal KR. A thermodynamic framework for the modeling of crystallizable shape memory polymers. Int J Eng Sci 2008;46:325-51.

[290] Qi HJ, Nguyen TD, Castroa F, Yakacki CM, ShandaSa R. Finite deformation thermo-mechanical behavior of thermally induced shape memory polymers. J Mech Phys Solids 2008;56:1730-51.

[291] Matveenko VP, Smetannikov OY, Trufanov NA, Shardakov IN. Models of thermomechanical behavior of polymeric materials undergoing glass transition. Acta Mech 2012;223:1261-84. 
[292] Westbrook KK, Parakh V, Chung T, Mather PT, Wan LC, Dunn ML, Qi HJ.

Constitutive modeling of shape memory effects in semicrystalline polymers with stretch induced crystallization. J Eng Mater Technol 2010;132:041010/1-9.

[293] Scott TF, Draughon RB, Bowman CN. Actuation in crosslinked polymers via photoinduced stress relaxation. Adv Mater 2006;18:2128-32.

[294] Bowman CN, Kloxin CJ. Covalent Adaptable Networks: Reversible Bond Structures Incorporated in Polymer Networks. Angew Chem Int Edit 2012;51:4272-4.

[295] Montarnal D, Capelot M, Tournilhac F, Leibler L. Silica-Like Malleable Materials from Permanent Organic Networks. Science 2011;334:965-8.

[296] Fringuelli F, Taticchi A, Wenkert E. Diels-Alder Reactions of Cycloalkenones in Organic-Synthesis - a Review. Org Prep Proced Int 1990;22:131-65.

[297] Oh T, Reilly M. Reagent-Controlled Asymmetric Diels-Alder Reactions - a Review. Org Prep Proced Int 1994;26:129-58.

[298] Hui CY, Long R. A constitutive model for the large deformation of a self-healing gel. Soft Matter 2012;8:8209-16.

[299] Long KN, Scott TF, Qi HJ, Bowman CN, Dunn ML. Photomechanics of light-activated polymers. J Mech Phys Solids 2009;57:1103-21.

[300] Sodhi JS, Rao IJ. Modeling the mechanics of light activated shape memory polymers. Int J Eng Sci 2010;48:1576-89.

[301] Long R, Qi HJ, Dunn ML. Thermodynamics and mechanics of photochemcially reacting polymers. J Mech Phys Solids 2013;61:2212-39.

[302] Ge Q, Luo XF, Rodriguez ED, Zhang X, Mather PT, Dunn ML, Qi HJ. Thermomechanical behavior of shape memory elastomeric composites. J Mech Phys Solids 2012;60:67-83.

[303] Ge Q, Luo XF, Iversen CB, Mather PT, Dunn ML, Qi HJ. Mechanisms of triple-shape polymeric composites due to dual thermal transitions. Soft Matter 2013;9:2212-23.

[304] Ge Q, Luo XF, Iversen CB, Nejad HB, Mather PT, Dunn ML, Qi HJ. A finite deformation thermomechanical constitutive model for triple shape polymeric composites based on dual thermal transitions. Int J Solids Struct 2014;51:2777-90. 
[305] Long R, Qi HJ, Dunn ML. Modeling the mechanics of covalently adaptable polymer networks with temperature-dependent bond exchange reactions. Soft Matter 2013;9:4083-96.

[306] Long KN. The mechanics of network polymers with thermally reversible linkages. J Mech Phys Solids 2014;63:386-411.

[307] Bonner M, de Oca HM, Brown M, Ward IM. A novel approach to predict the recovery time of shape memory polymers. Polymer 2010;51:1432-6.

[308] Ge Q, Yu K, Ding YF, Qi HJ. Prediction of temperature-dependent free recovery behaviors of amorphous shape memory polymers. Soft Matter 2012;8:11098-105.

[309] Castro F, Westbrook KK, Hermiller J, Ahn DU, Ding YF, Qi HJ. Time and Temperature Dependent Recovery of Epoxy-Based Shape Memory Polymers. J Eng Mater Technol 2011;133:021025/1-9.

[310] Yu K, McClung AJW, Tandon GP, Baur JW, Qi HJ. A thermomechanical constitutive model for an epoxy based shape memory polymer and its parameter identifications. Mech Time-Depend Mater 2014;18:453-74.

[311] Yu K, Ge Q, Qi HJ. Reduced time as a unified parameter determining fixity and free recovery of shape memory polymers. Nature Communications 2014;5:3066:1-9.

[312] Katzenberg F, Heuwers B, Tiller JC. Superheated Rubber for Cold Storage. Adv Mater 2011;23:1909-11.

[313] Heuwers B, Quitmann D, Katzenberg F, Tiller JC. Stress-induced melting of crystals in natural rubber: a new way to tailor the transition temperature of shape memory polymers. Macromol Rapid Commun 2012;33:1517-22.

[314] Quitmann D, Gushterov N, Sadowski G, Katzenberg F, Tiller JC. Environmental Memory of Polymer Networks under Stress. Adv Mater 2014;26:3441-4.

[315] Xie T, Li JJ, Zhao Q. Hidden thermoreversible actuation behavior of nafion and its morphological origin. Macromolecules 2014;47:1085-9.

[316] Sekitani T, Zschieschang U, Klauk H, Someya T. Flexible organic transistors and circuits with extreme bending stability. Nat Mater 2010;9:1015-22. 
[317] Yu ZB, Zhang QW, Li L, Chen Q, Niu XF, Liu J, Pei QB. Highly flexible silver nanowire electrodes for shape-memory polymer light-emitting diodes. Adv Mater 2011;23:664-8.

[318] Avendano-Bolivar A, Ware T, Arreaga-Salas D, Simon D, Voit W. Mechanical cycling stability of organic thin film transistors on shape memory polymers. Adv Mater $2013 ; 25: 3095-9$.

[319] Reeder J, Kaltenbrunner M, Ware T, Arreaga-Salas D, Avendano-Bolivar A, Yokota T, Inoue Y, Sekino M, Voit W, Sekitani T, Someya T. Mechanically adaptive organic transistors for implantable electronics. Adv Mater 2014;26:4967-73. 